\title{
TWO-PARAMETER QUANTUM ALGEBRAS, CANONICAL BASES AND CATEGORIFICATIONS
}

\author{
ZHAOBING FAN AND YIQIANG LI
}

\begin{abstract}
A theory of canonical basis for a two-parameter quantum algebra is developed in parallel with the one in one-parameter case. A geometric construction of the negative part of a two-parameter quantum algebra is given by using mixed perverse sheaves and Deligne's weight theory based on Lusztig's work [26. A categorification of the negative part of a two-parameter quantum algebra is provided. A two-parameter quantum algebra is shown to be a two-cocycle deformation, depending only on the second parameter, of its one-parameter analogue.
\end{abstract}

\section{INTRODUCTION}

One of the landmarks in Lie theory is the theory of canonical basis for a one-parameter quantum algebra developed by Lusztig in the ADE case in [24], and subsequently by Kashiwara [19] and Lusztig [25, 26] in general cases. It serves many times as a source of inspirations for the creation of a new direction in Lie theory such as cluster algebras [9] and the categorification program [6].

Among the various approaches to the theory of one-parameter quantum algebras and canonical bases, Lusztig's geometric construction of the negative part of a one-parameter quantum algebra by using perverse sheaves on representation varieties of a quiver plays a vital important role. In his geometric setting, many algebraic objects have a very natural interpretation from which several hidden structures are revealed. For example, the quantum parameter, the bar involution and canonical basis elements are incarnated as the shift functor, the Verdier duality functor and simple perverse sheaves arising from the geometric setting, respectively. The positivity of the structural constants of the canonical basis follows naturally from this geometric setting. If one reads Lusztig's work carefully, one notices that there is an ingredient, the Tate twist or the mixed structure, that Lusztig ignored in his geometric framework (see [26, 8.1.4]). It is desirable to see what Lusztig's geometric framework provides if the Tate twist is added.

In this paper, we construct an algebra from the mixed version of Lusztig's geometric framework by using mixed perverse sheaves on representation varieties of a quiver and Deligne's theory of weight, and we show that this algebra is isomorphic to the negative part of a twoparameter quantum algebra, in which the Tate twist corresponds to the second parameter.

From this geometric construction, we obtain several new features of a two-parameter quantum algebra. We are able to get a new presentation of generators and relations for

Date: October 3, 2018.

1991 Mathematics Subject Classification. 17B37, 16G20, 14R20, 14F43.

Key words and phrases. Two-parameter quantum algebra, mixed perverse sheaf, weight, canonical basis, categorification. 
a two-parameter quantum algebra determined by a certain matrix. This matrix serves as the generalized Cartan matrix and its symmetrization in the definition of a one-parameter quantum algebra. It is determined by a chosen orientation of a graph in symmetric cases. This presentation is new even in finite type. For example, the two parameters $v$ and $t$ we used in this paper are different from the one $(\alpha, \beta)$ used in literature in that they are related by $\alpha=v t$ and $\beta=v t^{-1}$. Furthermore, this presentation covers all Kac-Moody cases, unlike the one in literature which mainly studies finite type and some affine types. More importantly, it provides a new connection between a one-parameter quantum algebra and a two-parameter quantum algebra. As is shown in this paper, a two-parameter quantum algebra is a twococycle deformation, depending only on the second parameter, of its one-parameter analogue. As a consequence, if the underlying Cartan matrices of two two-parameter quantum algebras are the same, then they must be deformations of each other, and the deformation only depends on the second parameter. Last but not least, from the new presentation, we obtain a categorification of the negative part by utilizing Khovanov-Lauda's work [20] and [21].

From the geometric setting, we also obtain a basis for the negative part of a two-parameter quantum algebra consisting of simple perverse sheaves of weight zero. If one forgets the Tate twist, this basis is exactly the canonical basis in the one-parameter case. Moreover, the basis is a deformation of the canonical basis in the one-parameter case. In addition to its compatibility with the canonical basis in the one-parameter case, this basis admits many favorable properties such as integrality and positivity as does its one-parameter analogue. It also gives rise to a basis for each irreducible integrable highest weight module simultaneously. Moreover, if the underlying Cartan matrices of two two-parameter quantum algebras are the same, the canonical bases coincide under the deformation from one algebra to another (see Corollary 3). We follow Lusztig's approach in one-parameter case to give an algebraic characterization of this basis, and we call it the canonical basis of the negative half of a two-parameter quantum algebra. The characterization is in complete analogy with the one in one-parameter case. In particular, up to a sign, it is characterized by three properties: it is in the integral form of the negative half, it is bar invariant and it is almost orthonormal with respect to a bilinear form. This characterization is made possible by identifying the negative part with an analogue of Lusztig's algebra $\mathbf{f}$, which again comes from the geometric construction. In particular, both the bar involution and the bilinear form have natural interpretations in the geometric framework. The process to get rid of the sign is completely algebraic and follows closely Lusztig's argument in the one-parameter case.

In short, the mixed version of Lusztig's geometric framework is a natural geometric setting to study two-parameter quantum algebras. It provides a geometric construction of the negative part of a two-parameter quantum algebra. Based on the geometric work, we develop a canonical basis theory for the negative part of a two-parameter quantum algebra in an approach parallel with Lusztig's approach in the one-parameter case. Moreover, we show that the two-parameter quantum algebra is a two-cocycle deformation, depending only on the second parameter, of its one-parameter analogue. Finally, we give a categorification of the negative part in the sense of [20].

The intimate relationship revealed in this paper between a two-parameter quantum algebra and its one-parameter analogue by the specialization at $t=1$ and the deformation should play an important role in a forthcoming paper, where we shall continue to develop the 
canonical basis theory for the tensor product of integrable representations of two-parameter quantum algebras and two-parameter analogues of Lusztig's modified quantum algebras $\dot{\mathbf{U}}$.

A similar relationship between a quantum super algebra and the related two-parameter quantum algebra by a specialization at $t= \pm \mathbf{i}$, the imaginary unit, and a deformation with respect to the second parameter will be elaborated in [5]. Among others, we will provide a new categorification of a quantum super algebra different from the one in [14. This study, combining with the results in this paper and [5], should lead to interesting relations in the structural and representation theories of a one-parameter quantum algebra, its super and two-parameter analogues.

Meanwhile, the results obtained in this paper strongly suggest that a theory of crystal basis for two-parameter quantum algebras can be developed in parallel with the one in the one-parameter case by Kashiwara. We also hope that our work on two-parameter quantum algebras can shed some light on the open problem to develop a canonical basis theory for multiparameter quantum algebras.

Finally we remark that two-parameter quantum algebras have been studied from the early 1990s by various authors, see [7, 28, 2, 27, 12, 3, 17, 18, 16, 22, and the references therein. They also appear in I.B Frenkel's philosophical observations on the interactions of affinizations and quantizations of a Lie algebra ([8]). A new exciting development is Hill and Wang's categorification of the covering quantum algebra $\mathbf{f}^{\pi}$ which has two parameters with the second parameter $\pi$ subject to $\pi^{2}=1$ in [14. These work also inspire us during the formation of this paper. In Section 3.4, we compare the two-parameter quantum Serre relations with those available in the literature.

This paper is organized as follows. In Section 2, we review the geometric background, perverse sheaves and weight theory. We construct the algebra $\mathfrak{K}$ which is a geometric realization of $\mathfrak{f}$ for symmetric cases. In Section 3, we algebraically construct the algebra $\mathfrak{f}$, a two-parameter analogue of Lusztig's algebra $\mathbf{f}$, and compare it with various algebras in literatures. Those who are not interested in geometry can read this section directly. Section 4 provides two relations between the algebra $\mathfrak{f}$ and Lusztig's algebra $\mathbf{f}$ by specialization and deformation. These relations are generalized to the entire algebras. In Section 5, we present the algebraic characterization of the canonical basis of $\mathfrak{f}$, as well as that of the irreducible highest weight $U_{v, t}$ module $L(\lambda, \epsilon)$. Meanwhile, we show that the canonical basis of $\mathfrak{f}$ gets identified with the set of simple perverse sheaves of weight zero appeared in the geometric construction. In Section 6 , we give an algebraic categorification of $\mathfrak{f}$ which covers all symmetrizable cases.

Acknowledgements. We learned from Weiqiang Wang that two-parameter quantum algebras should be able to be realized geometrically. We thank Weiqiang for sharing with us his great idea and numerous discussions and comments on this project. We thank Zongzhu Lin for his valuable comments. We thank Jonathan Kujawa for bringing the paper [11] to our attention. Y. Li is partially supported by the NSF grant: DMS 1160351.

\section{Contents}

1. Introduction

2. The algebra $\mathfrak{K}$

3. The algebra $\mathfrak{f}$ 
4. Specialization and deformation $\quad 22$

5. The canonical basis $\quad 27$

6. A categorification of $\mathfrak{A} \mathfrak{f} \quad 30$

References $\quad 32$

\section{THE ALGEBRA $\mathfrak{K}$}

2.1. Review of mixed perverse sheaves. We review briefly the theory of mixed perverse sheaves. We refer to Chapter 8 in [26] and [1, 10] for more details.

Let $k$ be an algebraic closure of a finite field of $q$ elements. All algebraic varieties considered in this paper are over $k$. Let $l$ be a fixed prime number which is invertible in $k$, and $\overline{\mathbb{Q}}_{l}$ be an algebraic closure of the field $\mathbb{Q}_{l}$ of $l$-adic numbers. Denote by $\mathcal{D}(X)$ the bounded derived category of $\overline{\mathbb{Q}}_{l}$-constructible sheaves on the algebraic variety $X$. Let $\mathcal{M}(X)$ be the full subcategory of $\mathcal{D}(X)$ consisting of perverse sheaves on $X$. We denote by $\mathbf{1}_{X}$ the constant sheaf $\overline{\mathbb{Q}}_{l}$ on $X$, and simply by $\mathbf{1}$ if $X$ is obvious from the context.

Given any integer $n$, let $[n]: \mathcal{D}(X) \rightarrow \mathcal{D}(X)$ be the shift functor and $(n): \mathcal{D}(X) \rightarrow \mathcal{D}(X)$ be the Tate twist functor. Let ${ }^{p} H^{n}: \mathcal{D}(X) \rightarrow \mathcal{M}(X)$ be the perverse cohomology functor, and $\mathbb{D}: \mathcal{D}(X) \rightarrow \mathcal{D}(X)$ be the Verdier dual functor. Let $f: X \rightarrow Y$ be a morphism of algebraic varieties. There are functors $f^{*}, f^{!}: \mathcal{D}(Y) \rightarrow \mathcal{D}(X)$ and $f_{*}, f_{!}: \mathcal{D}(X) \rightarrow \mathcal{D}(Y)$. Moreover, if $f: X \rightarrow Y$ is a locally trivial principal $G$-bundle, then there is a well-defined functor $f_{\mathrm{b}}: \mathcal{M}_{G}(X)[n] \rightarrow \mathcal{M}(Y)[n+d]$ defined by $f_{b}(K)={ }^{p} H^{-n-d}\left(f_{*} K\right)[n+d]$, where $\mathcal{M}_{G}(X)$ is the full subcategory of $\mathcal{M}(X)$ consisting of all $G$-equivariant perverse sheaves and $d=\operatorname{dim} G$.

Let $\mathcal{D}_{m}(X)$ be the full subcategory of $\mathcal{D}(X)$ consisting of all mixed complexes, and $\mathcal{D}_{\leq w}(X)$ (resp. $\left.\mathcal{D}_{\geq w}(X)\right)$ be the full subcategory of $\mathcal{D}_{m}(X)$ consisting of all complexes whose $i$-th cohomology has weight $\leq w+i$ (resp. $\geq w+i)$. We simply denote by $\mathcal{D}_{\leq w}$ (resp. $\left.\mathcal{D}_{\geq w}\right)$ instead of $\mathcal{D}_{\leq w}(X)$ (resp. $\left.\mathcal{D}_{\geq w}(X)\right)$ if $X$ is obvious from the context.

A complex $K \in \mathcal{D}_{m}(X)$ is called pure of weight $w$ if $K \in \mathcal{D}_{\leq w}(X) \cap \mathcal{D}_{\geq w}(X)$. Denote by wt $(K)$ the weight of a pure complex $K$.

The functors $f^{*}, f_{*}, f^{!}, f_{!},[j], \otimes$ and Tate twist $(n)$ send mixed complexes to mixed complexes. We list some more properties as follows.

(a) Simple perverse sheaves are pure.

(b) If $X$ is smooth, then $\mathbf{1}_{X}$ is pure of weight 0 .

(c) If $K$ is a pure complex, then $\mathrm{wt}(K[1])=\mathrm{wt}(K)+1, \mathrm{wt}(K(1))=\mathrm{wt}(K)-2$.

(d) $\mathbb{D}(K[j])=\mathbb{D}(K)[-j], \mathbb{D}(K(n))=\mathbb{D}(K)(-n),{ }^{p} H^{n}(K)={ }^{p} H^{0}(K[n])$.

(e) $\mathbb{D}\left(\mathcal{D}_{\leq w}\right) \subset \mathcal{D}_{\geq-w}$ and $\mathbb{D}\left(\mathcal{D}_{\geq w}\right) \subset \mathcal{D}_{\leq-w}$. In particular, the Verdier dual sends pure complexes of weight $w$ to pure complexes of weight $-w$.

(f) The external tensor product functor $\square$ sends $\mathcal{D}_{\leq w_{1}} \times \mathcal{D}_{\leq w_{2}}$ (resp. $\mathcal{D}_{\geq w_{1}} \times \mathcal{D}_{\geq w_{2}}$ ) to $\mathcal{D}_{\leq w_{1}+w_{2}}\left(\right.$ resp. $\left.\mathcal{D}_{\geq w_{1}+w_{2}}\right)$. In particular, if $K, L$ are pure complexes, then $\operatorname{wt}(K \otimes L)=$ $\mathrm{wt}(K)+\mathrm{wt}(L)$.

(g) If $f: X \rightarrow Y$ is a morphism of varieties, then $f^{*}$ and $f_{\text {! }}$ preserve $\mathcal{D}_{\leq w}$ and $f_{*}$ and $f^{!}$ preserve $\mathcal{D}_{\geq w}$. In particular, if $f$ is a proper map, then $f_{!}$sends pure complexes of weight $w$ to pure complexes of weight $w$. 
(h) If $f: X \rightarrow Y$ is smooth with connected fibers of fiber dimension $d$, then $f^{*}[d]=$ $f^{!}[-d](-d)$ and $\mathbb{D} f^{*}(L)=f^{!}(\mathbb{D} L)$. Moreover, wt $\left(f^{*} K\right)=\operatorname{wt}(K)$ for any pure complex $K$.

2.2. The matrix $\Omega$. Let $I$ be a finite set. Throughout this paper, we fix a matrix $\Omega=$ $\left(\Omega_{i j}\right)_{i, j \in I}$ satisfying that

(a) $\Omega_{i i} \in \mathbb{Z}_{>0}, \Omega_{i j} \in \mathbb{Z}_{\leq 0}$ for all $i \neq j \in I$;

(b) $\frac{\Omega_{i j}+\Omega_{j i}}{\Omega_{i i}} \in \mathbb{Z}_{\leq 0}$ for all $i \neq j \in I$;

(c) the greatest common divisor of all $\Omega_{i i}$ is equal to 1 .

To $\Omega$, we associate the following three bilinear forms on $\mathbb{Z}^{I}$.

$$
\begin{aligned}
\langle i, j\rangle & =\Omega_{i j}, & & \forall i, j \in I . \\
{[i, j] } & =2 \delta_{i j} \Omega_{i i}-\Omega_{i j}, & & \forall i, j \in I . \\
i \cdot j & =\langle i, j\rangle+\langle j, i\rangle, & & \forall i, j \in I .
\end{aligned}
$$

Note that the form ". " satisfies the following properties:

$$
i \cdot i \in 2 \mathbb{Z}_{>0} \text { for any } i \in I \text { and } 2 \frac{i \cdot j}{i \cdot i} \in \mathbb{Z}_{\leq 0} \text { for any } i \neq j \text { in } I \text {. }
$$

It is a Cartan datum in Section 1.1.1 in [26].

The matrix $\Omega$ is called of symmetric type if $\Omega_{i i}=1, \forall i \in I$. In this case, the associated Cartan datum is of symmetric type.

For simplicity, we assume that $\Omega$ is of symmetric type in the rest of this section. To such a matrix, we associate a quiver whose vertex set is $I$, and whose arrow set consisting of $-\Omega_{i j}$ many arrows from vertex $i$ to vertex $j$ if $i \neq j$. By an abuse of notation, we denote by $\Omega$ the associated quiver. Since the matrix $\Omega$ is fixed, the quiver is thus fixed.

Note that the assignment of sending a matrix to its associated quiver defines a bijection between the set of such matrices and the set of quivers, up to isomorphisms.

2.3. The category $\mathfrak{Q}_{V}^{m}$. Let $V=\bigoplus_{i \in I} V_{i}$ be an $I$-graded $k$-vector space and $\underline{\operatorname{dim}} V=$ $\left(\operatorname{dim} V_{i}\right)_{i \in I} \in \mathbb{N}^{I}$. We define

$$
E_{V}=\bigoplus_{h \in \Omega} \operatorname{Hom}\left(V_{h^{\prime}}, V_{h^{\prime \prime}}\right), \quad G_{V}=\bigoplus_{i \in I} G L\left(V_{i}\right)
$$

where $h^{\prime}$ and $h^{\prime \prime}$ are the source and target of the arrow $h$ in $\Omega$, respectively. $G_{V}$ acts on $E_{V}$ by conjugation, i.e., $g x=x^{\prime}$ and $x_{h}^{\prime}=g_{h^{\prime \prime}} x_{h} g_{h^{\prime}}^{-1}$ for all $h \in \Omega$.

A subset $I^{\prime}$ in $I$ is said to be discrete if there is no arrow $h \in \Omega$ such that $\left\{h^{\prime}, h^{\prime \prime}\right\} \subset I^{\prime}$. We set $\operatorname{supp}(\nu)=\left\{i \in I \mid \nu_{i} \neq 0\right\}$, for any $\nu \in \mathbb{N}^{I}$. We call $\nu \in \mathbb{N}^{I}$ discrete if $\operatorname{supp}(\nu)$ is discrete.

Let $\underline{\nu}=\left(\nu^{1}, \nu^{2}, \cdots, \nu^{m}\right)$ be a sequence in $\mathbb{N}^{I}$ such that $\sum_{1 \leq l \leq m} \nu_{i}^{l}=\operatorname{dim} V_{i}$ and $\nu^{l}$ is discrete for all $l=1, \cdots, m$. A flag of type $\underline{\nu}$ in $V$ is a sequence

$$
f=\left(V=V^{0} \supset V^{1} \supset \cdots \supset V^{m}=0\right)
$$

of $I$-graded vector spaces such that $\underline{\operatorname{dim}} V^{l-1} / V^{l}=\nu^{l}, \forall 1 \leq l \leq m$. Let $\mathcal{F}_{\underline{\nu}}$ be the $k$-variety of all flags of type $\underline{\nu}$ in $V$. Let $\widetilde{\mathcal{F}}_{\underline{\nu}}=\left\{(x, f) \in E_{V} \times \mathcal{F}_{\underline{\nu}} \mid f\right.$ is $x$-stable $\}$, where $f$ is $x$-stable if $x_{h}\left(V_{h^{\prime}}^{l}\right) \subset V_{h^{\prime \prime}}^{l}$, for all $h \in \Omega$ and $1 \leq l \leq m$. 
Let $G_{V}$ act on $\mathcal{F}_{\underline{\nu}}$ by $g \cdot f \mapsto g f$, where $g f=\left(g V^{0} \supset g V^{1} \supset \cdots \supset g V^{m}=0\right)$, if $f=\left(V=V^{0} \supset V^{1} \supset \cdots \supset V^{m}=0\right)$. Let $G_{V}$ act diagonally on $\widetilde{\mathcal{F}}_{\underline{\nu}}$, i.e., $g \cdot(x, f) \mapsto(g x, g f)$. By Proposition 9.1.3 in [26], we have that $\widetilde{\mathcal{F}}_{\underline{\nu}}$ is a smooth irreducible variety of dimension

$$
d(\underline{\nu})=\sum_{i, l<l^{\prime}} \nu_{i}^{l^{\prime}} \nu_{i}^{l}+\sum_{h, l^{\prime}<l} \nu_{h^{\prime}}^{l^{\prime}} \nu_{h^{\prime \prime}}^{l}
$$

Moreover, the first projection map $\pi_{\underline{\nu}}: \widetilde{\mathcal{F}}_{\underline{\nu}} \rightarrow E_{V}$ is a proper $G_{V}$-equivariant morphism. As a consequence, the complex $\left(\pi_{\underline{\nu}}\right) ! \mathbf{1}_{\widetilde{\mathcal{F}}_{\underline{\nu}}}$ is a semisimple complex.

Lemma 1. The complex $\widetilde{L}_{\underline{\nu}}=\left(\pi_{\underline{\nu}}\right) ! \mathbf{1}_{\widetilde{\mathcal{F}}_{\underline{\underline{ }}}}$ is pure of weight 0 .

Proof. The lemma follows from (b) and Section 2.1(g).

We set

$$
\mathfrak{L}_{\underline{\nu}}=\widetilde{L}_{\underline{\nu}}[d(\underline{\nu})](d(\underline{\nu})) .
$$

Let $\mathfrak{Q}_{V}^{m}$ be the full subcategory of $\mathcal{D}_{m}\left(E_{V}\right)$ whose objects are isomorphic to finite direct sums of $L[d](n)$ for various $d \in \mathbb{Z}, n \in \frac{1}{2} \mathbb{Z}$ and various simple perverse sheaves $L$ satisfying the following property: $L$ is a direct summand of $\widetilde{L}_{\underline{\nu}}$ up to a shift and a Tate twist for some $\underline{\nu} \in \mathbb{N}^{I}$ such that $\sum_{1 \leq l \leq m} \nu_{i}^{l}=\operatorname{dim} V_{i}$.

We set $\mathfrak{Q}_{V}^{\leq w}=\mathfrak{Q}_{V}^{m} \bigcap \mathcal{D}_{\leq w}\left(E_{V}\right)$. This is the full subcategory of $\mathfrak{Q}_{V}^{m}$ consisting of mixed complexes whose $i$-th cohomology sheaf has weight $\leq w+i$ for all $i \in \mathbb{Z}$. Similarly, let $\mathfrak{Q}_{V}^{\geq w}=\mathfrak{Q}_{V}^{m} \bigcap \mathcal{D}_{\geq w}\left(E_{V}\right)$. We notice that $\mathfrak{Q}_{V}^{\leq 0} \cap \mathfrak{Q}_{V}^{\geq 0}$ is the same as $\mathcal{Q}_{V}$ defined in [26].

2.4. Additive generators. Let $\mathfrak{K}_{V}$ be the split Grothendieck group of the category $\mathfrak{Q}_{V}^{m}$. More precisely, $\mathfrak{K}_{V}$ is the abelian group generated by the isomorphism classes of objects in $\mathfrak{Q}_{V}^{m}$ which subjects to the following relation:

$$
\left[L \oplus L^{\prime}\right]=[L]+\left[L^{\prime}\right], \quad \forall L, L^{\prime} \in \mathfrak{Q}_{V}^{m} .
$$

Let $\mathfrak{M}_{V}$ be the split Grothendieck group of the full subcategory of $\mathfrak{Q}_{V}^{m}$ which consists of all direct sums of $\mathfrak{L}_{\underline{\nu}}$ for various $\underline{\nu}$ up to shifts and Tate twists. Similarly, let $\mathfrak{K}_{V}^{\leq w}$ (resp. $\mathfrak{K}_{V}^{\geq w}$ ) be the split Grothendieck group of the category $\mathfrak{Q}_{V}^{\leq w}$ (resp. $\mathfrak{Q}_{V}^{\geq w}$ ).

By an abuse of notation, we write $L$ instead of $[L]$ for elements in the Grothendieck group. Let $v$ and $t$ be two independent indeterminates and $\mathfrak{A}=\mathbb{Z}\left[v^{ \pm 1}, t^{ \pm 1}\right]$ be the subring of Laurent polynomials in $\mathbb{Q}(v, t)$. We define an $\mathfrak{A}$-action on $\mathfrak{K}_{V}$ by

$$
v \cdot L=L[1]\left(\frac{1}{2}\right), \quad t \cdot L=L\left(\frac{1}{2}\right) .
$$

Then $\mathfrak{K}_{V}$ is an $\mathfrak{A}$-module generated by the simple perverse sheaves of weight 0 in $\mathfrak{Q}_{V}^{m}$. Moreover, $\mathfrak{M}_{V}$ is an $\mathfrak{A}$-submodule of $\mathfrak{K}_{V}$ generated by $\mathfrak{L}_{\underline{\nu}}$.

Theorem 1. $\mathfrak{M}_{V}=\mathfrak{K}_{V}$ as $\mathfrak{A}$-modules, i.e., the set of $\mathfrak{L}_{\underline{\nu}}$ for various $\underline{\nu}$ contains an $\mathfrak{A}$-basis of $\mathfrak{K}_{V}$.

Proof. Recall that $\mathfrak{Q}_{V}^{\leq 0} \cap \mathfrak{Q}_{V}^{\geq 0}$ is the full subcategory of $\mathfrak{Q}_{V}^{m}$ consisting of pure complexes of weight 0 and $\operatorname{wt}\left(\widetilde{L}_{\underline{\nu}}\right)=0$ for any $\underline{\nu}$. By Proposition 12.6.2 in [26], $\widetilde{L}_{\underline{\nu}}$ are additive generators of $\mathfrak{K}_{V}^{\leq 0} \cap \mathfrak{K}_{V}^{\geq 0}$. Furthermore, by the definition of $\mathfrak{Q}_{V}^{m}$, for any element $K \in \mathfrak{K}_{V}$, there exist $B_{s} \in \mathfrak{K}_{V}^{\leq 0} \bigcap \mathfrak{K}_{V}^{\geq 0}$ such that $K=\sum_{s} v^{n_{s}} t^{m_{s}} B_{s}$ for some $n_{s}, m_{s}$. This implies that $\widetilde{L}_{\underline{\nu}}$ are 
additive generators of $\mathfrak{K}_{V}$. Moreover, $\mathfrak{L}_{\underline{\nu}}$ are also additive generators of $\mathfrak{K}_{V}$. This proves the theorem.

2.5. Induction functor. Let $W$ be an $I$-graded subspace of $V$ and $T=V / W$. Let $F=\{x \in$ $\left.E_{V} \mid x(W) \subset W\right\}$. For any $x \in F$, let $x_{W}$ be the restriction of $x$ to $W$ and $x_{T}: V / W \rightarrow V / W$ be the induced map of $x$ by passage to the quotient. Let $P$ be the stabilizer of $W$ in $G_{V}$ and $U$ be its unipotent radical. Consider Lusztig's diagram

$$
E_{T} \times E_{W} \stackrel{p_{1}}{\longleftarrow} G_{V} \times^{U} F \stackrel{p_{2}}{\longrightarrow} G_{V} \times^{P} F \stackrel{p_{3}}{\longrightarrow} E_{V}
$$

where $p_{1}(g, x)=\left(x_{T}, x_{W}\right), p_{2}(g, x)=(g, x), p_{3}(g, x)=g(\iota(x))$ and $\iota: F \rightarrow E_{V}$ is the embedding map. Let $G_{T} \times G_{W}$ act on $E_{T} \times E_{W}$ component-wise. We define

$$
\widetilde{\operatorname{Ind}}_{T, W}^{V} K=p_{3 !} p_{2 b} p_{1}^{*} K, \quad \forall K \in \mathcal{D}_{G_{T} \times G_{W}}\left(E_{T} \times E_{W}\right) .
$$

Proposition 1. If $K \in \mathfrak{Q}_{T}$ and $L \in \mathfrak{Q}_{W}$, then $\widetilde{\operatorname{Ind}}_{T, W}^{V}(K \otimes L) \in \mathfrak{Q}_{V}$. Moreover, if both $K$ and $L$ are pure, so is $\widetilde{\operatorname{Ind}}_{T, W}^{V}(K \otimes L)$, and its weight is equal to the sum of the weights of $K$ and $L$.

Proof. The proof of the first part of the proposition is similar to that of Lemma 9.2.3 in [26]. We only need to show the second part of the proposition. By Section 2.1(f) and (h), we have

$$
\mathrm{wt}\left(p_{2 b} p_{1}^{*}(K \otimes L)\right)=\mathrm{wt}(K)+\operatorname{wt}(L) .
$$

The proposition follows from the fact that $p_{3}$ is a proper map and Section 2.1(g).

We set

$$
\mathfrak{I n d}_{T, W}^{V}(K \otimes L)=\widetilde{\operatorname{Ind}}_{T, W}^{V}(K \otimes L)\left[d_{1}-d_{2}\right]\left(d_{1}-d_{2}\right),
$$

where $d_{1}$ (resp. $\left.d_{2}\right)$ is the fiber dimension of $p_{1}$ (resp. $\left.p_{2}\right)$ in Diagram (91).

Proposition 2. (a) If both $K$ and $L$ are pure, then

$$
\operatorname{wt}\left(\mathfrak{I n} \mathfrak{d}_{T, W}^{V}(K \otimes L)\right)=\operatorname{wt}(K)+\operatorname{wt}(L)-\left(d_{1}-d_{2}\right) .
$$

(b) $\mathfrak{I n} \mathfrak{d}_{T, W}^{V}\left(\mathfrak{L}_{\underline{\nu}^{\prime}} \otimes \mathfrak{L}_{\underline{\nu}^{\prime \prime}}\right)=\mathfrak{L}_{\underline{\nu}^{\prime} \underline{\nu}^{\prime \prime}}$, where $\mathfrak{L}_{\underline{\underline{\nu}}}$ is defined in (마).

Proof. Part (a) follows from Proposition 1 and (10). We now prove part (b).

$$
\begin{aligned}
& \mathfrak{I n d} d_{T, W}^{V}\left(\mathfrak{L}_{\underline{\nu}^{\prime}} \otimes \mathfrak{L}_{\underline{\nu}^{\prime \prime}}\right)=\widetilde{\operatorname{Ind}}_{T, W}^{V}\left(\widetilde{L}_{\underline{\underline{\prime}}^{\prime}} \otimes \widetilde{L}_{\underline{\nu}^{\prime \prime}}\right)\left[d\left(\underline{\nu}^{\prime}\right)+d\left(\underline{\nu}^{\prime \prime}\right)+d_{1}-d_{2}\right]\left(d\left(\underline{\nu}^{\prime}\right)+d\left(\underline{\nu}^{\prime \prime}\right)+d_{1}-d_{2}\right) \\
& =\widetilde{L}_{\underline{\nu}^{\prime} \underline{\prime}^{\prime \prime}}\left[d\left(\underline{\nu}^{\prime}\right)+d\left(\underline{\nu}^{\prime \prime}\right)+d_{1}-d_{2}\right]\left(d\left(\underline{\nu}^{\prime}\right)+d\left(\underline{\nu}^{\prime \prime}\right)+d_{1}-d_{2}\right) \\
& =\mathfrak{L}_{\underline{\nu}^{\prime} \underline{\nu}^{\prime \prime}}\left[d\left(\underline{\nu}^{\prime}\right)+d\left(\underline{\nu}^{\prime \prime}\right)+d_{1}-d_{2}-d\left(\underline{\nu}^{\prime} \underline{\nu}^{\prime \prime}\right)\right]\left(d\left(\underline{\nu}^{\prime}\right)+d\left(\underline{\nu}^{\prime \prime}\right)+d_{1}-d_{2}-d\left(\underline{\nu}^{\prime} \underline{\nu}^{\prime \prime}\right)\right) \text {. }
\end{aligned}
$$

Part (b) follows from the fact that $d\left(\underline{\nu}^{\prime}\right)+d\left(\underline{\nu}^{\prime \prime}\right)+d_{1}-d_{2}=d\left(\underline{\nu}^{\prime} \underline{\nu}^{\prime \prime}\right)$. 
2.6. The algebra $(\mathfrak{K}, \mathfrak{I n d})$. We notice that if $\underline{\operatorname{dim}} V_{1}=\underline{\operatorname{dim}} V_{2}$, then $E_{V_{1}}$ and $E_{V_{2}}$ are isomorphic. Moreover, the categories $\mathfrak{Q}_{V_{1}}^{m}$ and $\mathfrak{Q}_{V_{2}}^{m}$ are isomorphic. So we write $\mathfrak{Q}_{\nu}^{m}$ (resp. $\mathfrak{K}_{\nu}$ ) instead of $\mathfrak{Q}_{V}^{m}\left(\operatorname{resp} . \mathfrak{K}_{V}\right)$ if $\underline{\operatorname{dim}} V=\nu$. Now let

$$
\mathfrak{K}=\oplus_{\nu \in \mathbb{N} I} \mathfrak{K}_{\nu} .
$$

Define a multiplication on $\mathfrak{K}$ as follows.

$$
\mathfrak{I n d}: \mathfrak{K} \times \mathfrak{K} \rightarrow \mathfrak{K}, \quad(K, L) \mapsto \mathfrak{I n d}_{\tau, \omega}^{\nu}(K \otimes L),
$$

for any homogenous elements $K, L$ with $K \in \mathfrak{K}_{\tau}$ and $L \in \mathfrak{K}_{\omega}$.

Theorem 2. (1) The pair ( $\mathfrak{K}, \mathfrak{I n d})$ is an $\mathbb{N}^{I}$-graded associative $\mathfrak{A}$-algebra.

(2) All simple perverse sheaves of weight 0 in $\mathfrak{Q}_{\nu}^{m}$ for various $\nu$ form an $\mathfrak{A}$-basis of $\mathfrak{K}$ and a $\mathbb{Q}(v, t)$-basis of $\mathfrak{K} \otimes_{\mathfrak{A}} \mathbb{Q}(v, t)$.

Proof. (1) follows from Theorem 1, Proposition 2 and the additivity of $\mathfrak{I n d}$. (2) follows from the definition of $\mathfrak{K}$.

2.7. Defining relation. For any $k, n \in \mathbb{N}$ and $k \leq n$, we set

$$
\begin{array}{ll}
{[n]_{v}=\frac{v^{n}-v^{-n}}{v-v^{-1}}, \quad[n]_{v}^{!}=\prod_{k=1}^{n}[k]_{v}, \quad\left[\begin{array}{c}
n \\
k
\end{array}\right]_{v}=\frac{[n]_{v}^{!}}{[k]_{v}^{!}[n-k]_{v}^{!}},} \\
{[n]_{v, t}=\frac{(v t)^{n}-\left(v t^{-1}\right)^{-n}}{v t-\left(v t^{-1}\right)^{-1}}, \quad[n]_{v, t}^{!}=\prod_{k=1}^{n}[k]_{v, t}, \quad\left[\begin{array}{c}
n \\
k
\end{array}\right]_{v, t}=\frac{[n]_{v, t}^{!}}{[k]_{v, t}^{!}[n-k]_{v, t}^{!}} .}
\end{array}
$$

For any $k, n \in \mathbb{N}$ and $k \leq n$, we have

$$
[n]_{v, t}=t^{n-1}[n]_{v}, \quad[n]_{v, t}^{!}=t^{\frac{n(n-1)}{2}}[n]_{v}^{!}, \quad\left[\begin{array}{l}
n \\
k
\end{array}\right]_{v, t}=t^{k(n-k)}\left[\begin{array}{l}
n \\
k
\end{array}\right]_{v} .
$$

Example 1. Let $\Omega=[1]$. The associated quiver consists of a single vertex without any arrow. In this case, $E_{V}=\{\mathrm{pt}\}$ for any $V$. If $\underline{\nu}=n$, then $\mathcal{F}_{\underline{\underline{ }}}$ is also a point. Then $\widetilde{L}_{n}=\mathbf{1}_{E_{V}}$ for $V=k^{n}$ where $\widetilde{L}_{n}$ is defined in Lemma 1,

If $\underline{\nu}=(n, 1)$, then $\mathcal{F}_{\underline{\nu}}=\left\{f=\left(0 \subset V^{1} \subset V\right) \mid \operatorname{dim} V^{1}=1\right\}$ is the Grassmannian $\operatorname{Gr}(1, n+1)$. By Lemma 5.4 .12 in [1], $\widetilde{L}_{(n, 1)}=\oplus_{i=0}^{n} \mathbf{1}_{E_{V}}[-2 i](-i)$. By ([6) $)$, we have

$$
\mathfrak{L}_{(n, 1)}=\bigoplus_{i=0}^{n} \mathbf{1}_{E_{V}}[n-2 i](n-i)=\sum_{0 \leq i \leq n} v^{n-2 i} t^{n} \mathfrak{L}_{n+1}=[n+1]_{v, t} \mathfrak{L}_{n+1} .
$$

In other words,

$$
\mathfrak{L}_{n} \cdot \mathfrak{L}_{1}=[n+1]_{v, t} \mathfrak{L}_{n+1} .
$$

Example 2. Let $\Omega=\left[\begin{array}{cc}1 & -a^{\prime} \\ -a^{\prime \prime} & 1\end{array}\right]$, where $a^{\prime}, a^{\prime \prime} \geq 0$. The associated quiver has two vertices, say $i$ and $j$. Let $\Omega^{\prime}$ (resp. $\Omega^{\prime \prime}$ ) be the set of arrows from $i$ to $j$ (resp. from $j$ to $i$ ). Then $a^{\prime}=\# \Omega^{\prime}, a^{\prime \prime}=\# \Omega^{\prime \prime}$. Set $N=a^{\prime}+a^{\prime \prime}$.

Fix a vector space $V=V_{i} \oplus V_{j}$ such that $\operatorname{dim} V_{i}=1$ and $\operatorname{dim} V_{j}=N+1$. For any $p=0,1, \cdots, N+1$, let

$$
\widetilde{S}_{p}=\left\{(x, W) \in E_{V} \times \operatorname{Gr}\left(p^{\prime}, V_{j}\right) \mid x_{h}\left(V_{i}\right) \subset W, \text { if } h \in \Omega^{\prime} ;\left.x_{h}\right|_{W}=0 \text { if } h \in \Omega^{\prime \prime}\right\},
$$


where $p^{\prime}=N+1-p$ and $\operatorname{Gr}\left(p^{\prime}, V_{j}\right)$ is the Grassmannian of all $p^{\prime}$-dimensional subspaces in $V_{j}$. Let $\pi\left(\widetilde{S}_{p}\right)$ be the image of the first projection map $\pi: \widetilde{S}_{p} \rightarrow E_{V}$. Let

$$
I_{p}^{\prime}=\pi_{!} \mathbf{1}_{\widetilde{S}_{p}}\left[\operatorname{dim} \widetilde{S}_{p}\right]\left(\frac{1}{2} \operatorname{dim} \widetilde{S}_{p}\right) .
$$

This is a semisimple complex and pure of weight 0 since $\pi$ is a proper map. Let $I_{p}:=$ $I C\left(\pi\left(\widetilde{S}_{p}\right), 1\right)$ be the intersection cohomology complex of weight 0 on $E_{V}$ determined by $\pi\left(\widetilde{S}_{p}\right)$ and the constant sheaf on its smooth part. From Proposition 9.4 in [25], we have

Lemma 2. (a) $I_{0}^{\prime}=I_{0}, I_{N+1}^{\prime}=I_{N+1}$,

(b) $I_{p}^{\prime}=I_{p} \oplus I_{p-1}$ if $1 \leq p \leq a^{\prime \prime} ; \quad I_{p}^{\prime}=I_{p} \oplus I_{p+1}$ if $a^{\prime \prime}+1 \leq p \leq N$,

(c) $I_{a^{\prime \prime}}=I_{a^{\prime \prime}+1}$,

(d) $\operatorname{dim}\left(\widetilde{S}_{p}\right)=\left(p+a^{\prime}\right)(N+1-p)+a^{\prime \prime} p$.

If $\underline{\nu}=(j, j, \cdots, j)$ for $p$ iterated $j$, then we denote by $\mathfrak{L}_{j^{p}}$ instead of $\mathfrak{L}_{\underline{\nu}}$. By Proposition 2. we have $\mathfrak{L}_{j^{p}, i, j^{p^{\prime}}}=[p]_{v, t}^{!}\left[p^{\prime}\right]_{v, t}^{!} \mathfrak{L}_{p j, i, p^{\prime} j}$. By (마) and the definition of $I_{p}^{\prime}$, we further have $\mathfrak{L}_{j^{p}, i, j^{p^{\prime}}}=[p]_{v, t}^{!}\left[p^{\prime}\right]_{v, t}^{!} I_{p}^{\prime}\left(\frac{1}{2} \operatorname{dim}\left(\widetilde{S}_{p}\right)\right)$. Thus we have

$$
\begin{aligned}
& \frac{1}{[N+1]_{v, t}^{!}} \sum_{0 \leq p \leq N+1}(-1)^{p} t^{-p\left(p^{\prime}-a^{\prime}+a^{\prime \prime}\right)}\left[\begin{array}{c}
N+1 \\
p
\end{array}\right]_{v, t} \mathfrak{L}_{j^{p}, i, j^{p^{\prime}}} \\
= & \sum_{0 \leq p \leq N+1}(-1)^{p} t^{-p\left(p^{\prime}-a^{\prime}+a^{\prime \prime}\right)} t^{\left(p+a^{\prime}\right) p^{\prime}+a^{\prime \prime} p} I_{p}^{\prime}=\sum_{0 \leq p \leq N+1}(-1)^{p} t^{a^{\prime}(N+1)} I_{p}^{\prime}=0,
\end{aligned}
$$

where the last equality follows from Lemma 2, By combining Examples 1 and 2, we have

Proposition 3. The following relations satisfy in $\mathfrak{K}$ associated to any quiver $\Omega$.

$$
\begin{gathered}
\mathfrak{L}_{n i} \cdot \mathfrak{L}_{i}=[n+1]_{v, t} \mathfrak{L}_{(n+1) i}, \quad \forall i \in I . \\
\sum_{p=0}^{i \cdot j+1}(-1)^{p} t^{-p\left(p^{\prime}-\langle i, j\rangle+\langle j, i\rangle\right)}\left[\begin{array}{c}
i \cdot j+1 \\
p
\end{array}\right]_{v, t} \mathfrak{L}_{j^{p}, i, j^{p^{\prime}}}=0, \quad \forall i \neq j \in I .
\end{gathered}
$$

2.8. Restriction functor. Consider the following diagram

$$
E_{T} \times E_{W} \stackrel{\kappa}{\longleftarrow} F \stackrel{\iota}{\longrightarrow} E_{V}
$$

where $\iota$ is an embedding and $\kappa(x)=\left(x_{T}, x_{W}\right)$. We define

$$
\widetilde{\operatorname{Res}}_{T, W}(L)=\kappa_{!} \iota^{*} L, \quad \forall L \in \mathcal{D}\left(E_{V}\right) .
$$

Proposition 4. $\widetilde{\operatorname{Res}}_{T, W}^{V}\left(\widetilde{L}_{\underline{\nu}}\right)=\oplus_{\underline{\tau}, \underline{\omega}} \widetilde{L}_{\underline{\tau}} \otimes \widetilde{L}_{\underline{\omega}}[-2 M(\underline{\tau}, \underline{\omega})](-M(\underline{\tau}, \underline{\omega}))$, where

$$
M(\underline{\tau}, \underline{\omega})=\sum_{h ; l^{\prime}<l} \tau_{h^{\prime}}^{l^{\prime}} \omega_{h^{\prime \prime}}^{l}+\sum_{i ; l<l^{\prime}} \tau_{i}^{l^{\prime}} \omega_{i}^{l}
$$

and the direct sum is taken over all $\underline{\tau}$ and $\underline{\omega}$ such that $\tau^{l}+\omega^{l}=\nu^{l}, \sum_{l} \omega_{i}^{l}=\operatorname{dim} W_{i}$ and $\sum_{l} \tau_{i}^{l}=\operatorname{dim} T_{i}$

Proof. By 9.2.6 (b) in [26], we have $\widetilde{\operatorname{Res}}_{T, W}^{V}\left(\widetilde{L}_{\underline{\nu}}\right) \simeq \oplus_{\underline{\tau}, \underline{\omega}}\left(\widetilde{L}_{\underline{\tau}} \otimes \widetilde{L}_{\underline{\omega}}\right)[-2 M(\underline{\tau}, \underline{\omega})]$ up to a Tate twist. It is enough to check that the weights of the two complexes on both sides in the 
proposition are the same. Let $\iota^{\prime}: E_{T} \times E_{W} \rightarrow F$ be the embedding map. By (1) in [4], we have

$$
\kappa_{!} \iota^{*} L \simeq\left(\iota^{\prime}\right)^{!}(\iota)^{*} L, \quad \forall L \in \mathcal{D}_{G_{V}}\left(E_{V}\right) .
$$

Note that the functor $\left(\iota^{\prime}\right)^{!}(\iota)^{*} L$ is the hyperbolic localization functor. By Lemma 1 and Theorem 8 in [4, the weights of both complexes in the proposition are zero. The proposition follows.

For any $L \in \mathcal{D}_{G_{V}}\left(E_{V}\right)$, we define

$$
\mathfrak{R e s}_{T, W}^{V}(L)=\widetilde{\operatorname{Res}}_{T, W}^{V}(L)\left[d_{1}-d_{2}-2 \operatorname{dim}\left(G_{V} / P\right)\right]\left(-\operatorname{dim}\left(G_{V} / P\right)\right),
$$

where $d_{1}$ and $d_{2}$ are the same as those in (10). By Theorem 8 in [4], Proposition 4, (6) and (17), we have the following corollary.

Corollary 1. $\mathfrak{R e s}_{T, W}^{V}\left(\mathfrak{L}_{\underline{\nu}}\right)=\oplus_{\underline{\tau}, \underline{\omega}} \mathfrak{L}_{\underline{\tau}} \otimes \mathfrak{L}_{\underline{\omega}}\left[M^{\prime}(\underline{\tau}, \underline{\omega})\right]\left(M^{\prime \prime}(\underline{\tau}, \underline{\omega})\right)$, where

$$
\begin{gathered}
M^{\prime}(\underline{\tau}, \underline{\omega})=d_{1}-d_{2}-2 \operatorname{dim}\left(G_{V} / P\right)+d(\underline{\nu})-d(\underline{\tau})-d(\underline{\omega})-2 M(\underline{\tau}, \underline{\omega}), \\
M^{\prime \prime}(\underline{\tau}, \underline{\omega})=d(\underline{\nu})-d(\underline{\tau})-d(\underline{\omega})-\operatorname{dim}\left(G_{V} / P\right)-M(\underline{\tau}, \underline{\omega}),
\end{gathered}
$$

and the direct sum is taken over all $\underline{\tau}$ and $\underline{\omega}$ such that $\tau^{l}+\omega^{l}=\nu^{l}, \sum_{l} \omega_{i}^{l}=\operatorname{dim} W_{i}$ and $\sum_{l} \tau_{i}^{l}=\operatorname{dim} T_{i}$. Moreover, if $L$ is a pure complex in $\mathcal{D}_{G_{V}}\left(E_{V}\right)$, then

$$
\operatorname{wt}\left(\mathfrak{R e s}_{T, W}^{V} L\right)=\operatorname{wt}(L)+d_{1}-d_{2} .
$$

2.9. Coalgebra structure. Define an $\mathfrak{A}$-linear map $\mathfrak{r}: \mathfrak{K} \rightarrow \mathfrak{K} \otimes \mathfrak{K}$ by

$$
K \mapsto \oplus_{\tau, \omega} \mathfrak{R e s} \mathfrak{s}_{\tau, \omega}^{\nu} K,
$$

for any homogenous element $K \in \mathfrak{K}_{\nu}$, where the direct sum runs through all $\tau, \omega \in \mathbb{N}^{I}$ such that $\tau+\omega=\nu$.

Define a multiplication on $\mathfrak{K} \otimes \mathfrak{K}$ as follows.

$$
(x \otimes y)\left(x^{\prime} \otimes y^{\prime}\right)=v^{-\left|x^{\prime}\right| \cdot|y|} t^{\left\langle\left|x^{\prime}\right|,|y|\right\rangle-\left\langle|y|,\left|x^{\prime}\right|\right\rangle} x x^{\prime} \otimes y y^{\prime},
$$

for homogenous elements $x, y, x^{\prime}$ and $y^{\prime}$, where $|x|$ is the grading of $x$ and $\langle$,$\rangle is defined in$ (1).

Proposition 5. $\mathfrak{r}: \mathfrak{K} \rightarrow \mathfrak{K} \otimes \mathfrak{K}$ is an algebra homomorphism, where the algebra structure on $\mathfrak{K} \otimes \mathfrak{K}$ is defined in (20).

Proof. By Theorem 1 and Proposition 2, it is enough to show that

$$
\mathfrak{r}\left(\mathfrak{L}_{\underline{\nu}^{\prime} \underline{\nu}^{\prime \prime}}\right)=\mathfrak{r}\left(\mathfrak{L}_{\underline{\underline{\nu}}^{\prime}}\right) \mathfrak{r}\left(\mathfrak{L}_{\underline{\underline{\nu}}^{\prime \prime}}\right) \text { for any } \underline{\nu}^{\prime}, \underline{\nu}^{\prime \prime} \in \mathbb{N}^{I}
$$

By Corollary 1 ,

$$
\mathfrak{r}\left(\mathfrak{L}_{\underline{\nu}^{\prime}}\right)=\sum \mathfrak{L}_{{\underline{\tau^{\prime}}}^{\prime}} \otimes \mathfrak{L}_{\underline{\omega}^{\prime}}\left[M^{\prime}\left(\underline{\tau}^{\prime}, \underline{\omega}^{\prime}\right)\right]\left(M^{\prime \prime}\left(\underline{\tau}^{\prime}, \underline{\omega}^{\prime}\right)\right)
$$

where the sum is taken over all $\underline{\tau}^{\prime}$ and $\underline{\omega}^{\prime}$ such that $\tau^{\prime l}+\omega^{\prime l}=\nu^{\prime l}$ for all $l=1, \cdots, m$.

Similarly, we have

$$
\mathfrak{r}\left(\mathfrak{L}_{\underline{\underline{\nu}}^{\prime \prime}}\right)=\sum \mathfrak{L}_{{\underline{\tau^{\prime \prime}}}^{\prime \prime}} \otimes \mathfrak{L}_{\underline{\omega}^{\prime \prime}}\left[M^{\prime}\left(\underline{\tau}^{\prime \prime}, \underline{\omega}^{\prime \prime}\right)\right]\left(M^{\prime \prime}\left(\underline{\tau}^{\prime \prime}, \underline{\omega}^{\prime \prime}\right)\right),
$$


where the sum is taken over all $\underline{\tau}^{\prime \prime}$ and $\underline{\omega}^{\prime \prime}$ such that $\tau^{\prime \prime l}+\omega^{\prime \prime l}=\nu^{\prime \prime l}$ for all $l=m+1, \cdots, m+n$. By (8), we can rewrite (20) as follows.

$$
(x \otimes y)\left(x^{\prime} \otimes y^{\prime}\right)=x x^{\prime} \otimes y y^{\prime}\left[-|y| \cdot\left|x^{\prime}\right|\right]\left(-\left\langle|y|,\left|x^{\prime}\right|\right\rangle\right),
$$

where $|y| \cdot\left|x^{\prime}\right|=\left\langle\left|x^{\prime}\right|,|y|\right\rangle+\left\langle|y|,\left|x^{\prime}\right|\right\rangle$ is a symmetric bilinear form. Therefore,

$$
\mathfrak{r}\left(\mathfrak{L}_{\underline{\underline{\nu}}^{\prime}}\right) \mathfrak{r}\left(\mathfrak{L}_{\underline{\underline{\nu}}^{\prime \prime}}\right)=\sum \mathfrak{L}_{\underline{\tau}^{\prime} \underline{\tau}^{\prime \prime}} \otimes \mathfrak{L}_{\underline{\omega}^{\prime} \underline{\omega}^{\prime \prime}}\left[N^{\prime}\left(\underline{\tau}^{\prime} \underline{\tau}^{\prime \prime}, \underline{\omega}^{\prime} \underline{\omega}^{\prime \prime}\right)\right]\left(N^{\prime \prime}\left(\underline{\tau}^{\prime} \underline{\tau}^{\prime \prime}, \underline{\omega}^{\prime} \underline{\omega}^{\prime \prime}\right)\right),
$$

where the sum is taken over all $\underline{\tau}^{\prime}, \underline{\omega}^{\prime}, \underline{\tau}^{\prime \prime}$ and $\underline{\omega}^{\prime \prime}$ such that $\tau^{\prime l}+\omega^{\prime l}=\nu^{\prime l}$ for all $l=1, \cdots, m$ and $\tau^{\prime \prime l}+\omega^{\prime \prime l}=\nu^{\prime \prime l}$ for all $l=m+1, \cdots, m+n$.

$$
\begin{gathered}
N^{\prime}\left(\underline{\tau}^{\prime} \underline{\tau}^{\prime \prime}, \underline{\omega}^{\prime} \underline{\omega}^{\prime \prime}\right)=M^{\prime}\left(\underline{\tau}^{\prime}, \underline{\omega}^{\prime}\right)+M^{\prime}\left(\underline{\tau}^{\prime \prime}, \underline{\omega}^{\prime \prime}\right)-\left|\underline{\tau}^{\prime \prime}\right| \cdot\left|\underline{\omega}^{\prime}\right|, \text { and } \\
N^{\prime \prime}\left(\underline{\tau}^{\prime} \underline{\tau}^{\prime \prime}, \underline{\omega}^{\prime} \underline{\omega}^{\prime \prime}\right)=M^{\prime \prime}\left(\underline{\tau}^{\prime}, \underline{\omega}^{\prime}\right)+M^{\prime \prime}\left(\underline{\tau}^{\prime \prime}, \underline{\omega}^{\prime \prime}\right)-\left\langle\left|\underline{\omega}^{\prime}\right|,\left|\underline{\tau}^{\prime \prime}\right|\right\rangle .
\end{gathered}
$$

On the other hand, we have

$$
\mathfrak{r}\left(\mathfrak{L}_{\underline{\underline{\nu}}^{\prime} \underline{\underline{\nu}}^{\prime \prime}}\right)=\sum \mathfrak{L}_{\underline{\tau}^{\prime} \underline{\tau}^{\prime \prime}} \otimes \mathfrak{L}_{\underline{\omega}^{\prime} \underline{\omega}^{\prime \prime}}\left[M^{\prime}\left(\underline{\tau}^{\prime} \underline{\tau}^{\prime \prime}, \underline{\omega}^{\prime} \underline{\omega}^{\prime \prime}\right)\right]\left(M^{\prime \prime}\left(\underline{\tau}^{\prime} \underline{\tau}^{\prime \prime}, \underline{\omega}^{\prime} \underline{\omega}^{\prime \prime}\right)\right) .
$$

By comparing (21) with (22), it remains to show that

$$
M^{\prime}\left(\underline{\tau}^{\prime} \underline{\tau}^{\prime \prime}, \underline{\omega}^{\prime} \underline{\omega}^{\prime \prime}\right)=N^{\prime}\left(\underline{\tau}^{\prime} \underline{\tau}^{\prime \prime}, \underline{\omega}^{\prime} \underline{\omega}^{\prime \prime}\right) \text {, and } M^{\prime \prime}\left(\underline{\tau}^{\prime} \underline{\tau}^{\prime \prime}, \underline{\omega}^{\prime} \underline{\omega}^{\prime \prime}\right)=N^{\prime \prime}\left(\underline{\tau}^{\prime} \underline{\tau}^{\prime \prime}, \underline{\omega}^{\prime} \underline{\omega}^{\prime \prime}\right) .
$$

The proof of the first one is the same as that of Lemma 13.1.5 in [26]. We only need to show that the second one holds.

By equations (18) and (19), we have

$$
\begin{aligned}
& M^{\prime \prime}\left(\underline{\tau}^{\prime} \underline{\tau}^{\prime \prime}, \underline{\omega}^{\prime} \underline{\omega}^{\prime \prime}\right)-N^{\prime \prime}\left(\underline{\tau}^{\prime} \underline{\tau}^{\prime \prime}, \underline{\omega}^{\prime} \underline{\omega}^{\prime \prime}\right) \\
= & d\left(\underline{\nu}^{\prime} \underline{\nu}^{\prime \prime}\right)-d\left(\underline{\tau}^{\prime} \tau^{\prime \prime}\right)-d\left(\underline{\omega}^{\prime} \underline{\omega}^{\prime \prime}\right)-\operatorname{dim}\left(G_{V} / P\right)_{\underline{\tau}^{\prime} \underline{\tau}^{\prime \prime}, \underline{\omega}^{\prime} \underline{\omega}^{\prime \prime}}-M\left(\underline{\tau}^{\prime} \underline{\tau}^{\prime \prime}, \underline{\omega}^{\prime} \underline{\omega}^{\prime \prime}\right) \\
- & \left(d\left(\underline{\nu}^{\prime}\right)-d\left(\underline{\tau}^{\prime}\right)-d\left(\underline{\omega}^{\prime}\right)-\operatorname{dim}\left(G_{V} / P\right)_{\underline{\tau}^{\prime}, \underline{\omega}^{\prime}}-M\left(\underline{\tau}^{\prime}, \underline{\omega}^{\prime}\right)\right) \\
- & \left(d\left(\underline{\nu}^{\prime \prime}\right)-d\left(\underline{\tau}^{\prime \prime}\right)-d\left(\underline{\omega}^{\prime \prime}\right)-\operatorname{dim}\left(G_{V} / P\right)_{\underline{\tau}^{\prime \prime}, \underline{\omega}^{\prime \prime}}-M\left(\underline{\tau}^{\prime \prime}, \underline{\omega}^{\prime \prime}\right)\right)+\left\langle\left|\underline{\omega}^{\prime}\right|,\left|\underline{\tau}^{\prime \prime}\right|\right\rangle,
\end{aligned}
$$

where $\operatorname{dim}\left(G_{V} / P\right)_{\underline{\tau}, \underline{\omega}}=\sum_{i} \underline{\tau}_{i} \underline{\omega}_{i}$ and $\underline{\tau}_{i}=\sum_{l} \tau_{i}^{l}$. Moreover,

$$
\operatorname{dim}\left(G_{V} / P\right)_{{\underline{\tau^{\prime}}}^{\prime}{\underline{\tau^{\prime}}}^{\prime} \underline{\omega}^{\prime} \underline{\omega}^{\prime \prime}}-\operatorname{dim}\left(G_{V} / P\right)_{{\underline{\tau^{\prime}}, \underline{\omega^{\prime}}}}-\operatorname{dim}\left(G_{V} / P\right)_{{\underline{\tau^{\prime \prime}}}^{\prime \prime} \underline{\omega}^{\prime \prime}}=\sum_{i} \tau_{i}^{\prime} \omega_{i}^{\prime \prime}+\tau_{i}^{\prime \prime} \omega_{i}^{\prime} .
$$

In general, if $\underline{\nu}=\underline{\tau}+\underline{\omega}$, we have

$$
d(\underline{\nu})-d(\underline{\tau})-d(\underline{\omega})=\sum_{h ; l^{\prime}<l} \tau_{h^{\prime}}^{l^{\prime}} \omega_{h^{\prime \prime}}^{l}+\omega_{h^{\prime}}^{l^{\prime}} \tau_{h^{\prime \prime}}^{l}+\sum_{i ; l<l^{\prime}} \tau_{i}^{l^{\prime}} \omega_{i}^{l}+\omega_{i}^{l^{\prime}} \tau_{i}^{l} .
$$

Hence,

$$
\begin{aligned}
& d\left(\underline{\nu}^{\prime} \underline{\nu}^{\prime \prime}\right)-d\left(\underline{\tau}^{\prime} \underline{\tau}^{\prime \prime}\right)-d\left(\underline{\omega}^{\prime} \underline{\omega}^{\prime \prime}\right)-\left(d\left(\underline{\nu}^{\prime}\right)-d\left(\underline{\tau}^{\prime}\right)-d\left(\underline{\omega}^{\prime}\right)\right) \\
& -\left(d\left(\underline{\nu}^{\prime \prime}\right)-d\left(\underline{\tau}^{\prime \prime}\right)-d\left(\underline{\omega}^{\prime \prime}\right)\right)=\sum_{h} \tau_{h^{\prime}}^{\prime} \omega_{h^{\prime \prime}}^{\prime \prime}+\omega_{h^{\prime}}^{\prime} \tau_{h^{\prime \prime}}^{\prime \prime}+\sum_{i} \tau_{i}^{\prime \prime} \omega_{i}^{\prime}+\omega_{i}^{\prime \prime} \tau_{i}^{\prime},
\end{aligned}
$$

where $\tau_{h^{\prime}}^{\prime}=\sum_{l} \tau_{h^{\prime}}^{\prime l}$. By (16), we have

$$
M\left(\underline{\tau}^{\prime} \underline{\tau}^{\prime \prime}, \underline{\omega}^{\prime} \underline{\omega}^{\prime \prime}\right)-M\left(\underline{\tau}^{\prime}, \underline{\omega}^{\prime}\right)-M\left(\underline{\tau}^{\prime \prime}, \underline{\omega}^{\prime \prime}\right)=\sum_{h} \tau_{h^{\prime}}^{\prime} \omega_{h^{\prime \prime}}^{\prime \prime}+\sum_{i} \tau_{i}^{\prime \prime} \omega_{i}^{\prime} .
$$

By (25), (26) and (27), the right hand side of (24) is 0 . This shows that (23) holds. 
2.10. Bar involution. Given any pure complex $K \in \mathfrak{Q}_{V}^{m}$, let

$$
\mathfrak{D}(K)=(\mathbb{D} K)(-\mathrm{wt}(K)) .
$$

Since objects in $\mathfrak{Q}_{V}^{m}$ are semisimple, this defines a functor $\mathfrak{D}: \mathfrak{Q}_{V}^{m} \rightarrow \mathfrak{Q}_{V}^{m}$. We notice that $\mathfrak{D}^{2}$ is the identity functor. By (6) and Section $2.1(\mathrm{~d}), \mathbb{D} \mathfrak{L}_{\nu}=\mathfrak{L}_{\nu}(-d(\nu))$ and $\operatorname{wt}\left(\mathfrak{L}_{\nu}\right)=-d(\nu)$. So we have

$$
\mathfrak{D}\left(\mathfrak{L}_{\nu}\right)=\mathfrak{L}_{\nu} .
$$

Proposition 6. If both $K \in \mathfrak{Q}_{T}^{m}$ and $L \in \mathfrak{Q}_{W}^{m}$ are pure, then we have

$$
\mathfrak{D} \circ \mathfrak{I n d}(K \otimes L)=\mathfrak{I n} \mathfrak{d} \circ \mathfrak{D}(K \otimes L) .
$$

Proof. By Proposition 9.2.5 in 26, it is enough to check that the weights on both sides equal. The proposition follows from the fact that $\mathfrak{D}$ preserves the weights of pure complexes.

Define an involution ${ }^{-}: \mathfrak{K} \rightarrow \mathfrak{K}$ by

$$
v \mapsto v^{-1}, t \mapsto t \text {, and } K \mapsto \mathfrak{D}(K) \text {. }
$$

Proposition 7. The map ${ }^{-}: \mathfrak{K} \rightarrow \mathfrak{K}$ is a $\mathbb{Q}(t)$-algebra involution.

Proof. By Proposition 2, Theorem 1 and (28), it is enough to check that

$$
\overline{v \cdot K}=v^{-1} \cdot \bar{K} \text {, and } \overline{t \cdot K}=t \cdot \bar{K}, \forall K \in \mathfrak{K} \text {. }
$$

By the definition of "-", we can assume that $K$ is the isomorphism class of a pure complex. Set $\operatorname{wt}(K)=w$. Then we have

$$
\overline{v \cdot K}=\overline{K[1]\left(\frac{1}{2}\right)}=\left(\mathbb{D}\left(K[1]\left(\frac{1}{2}\right)\right)\right)(-w)=(\mathbb{D}(K))[-1]\left(-w-\frac{1}{2}\right) .
$$

On the other hand,

$$
v^{-1} \cdot \bar{K}=v^{-1} \cdot(\mathbb{D} K)(-w)=(\mathbb{D}(K))[-1]\left(-w-\frac{1}{2}\right) .
$$

Similarly, one can check that $\overline{t \cdot K}=t \cdot \bar{K}$.

2.11. Bilinear form. Recall that for any two $G$-equivariant semisimple complexes $K, L$ on $X$, one can define a number, $d_{j}(K, L) \in \mathbb{Z}_{\geq 0}$, for any $j \in \mathbb{Z}$ which Lusztig denotes by $d_{j}(X, G ; K, L)([13,23,26])$. Given any two pure complexes $K, L \in \mathfrak{Q}_{V}^{m}$, we define

$$
(K, L)=\sum_{j \in \mathbb{Z}} d_{j}(K, L) v^{-j} t^{-(\operatorname{wt}(K)+\operatorname{wt}(L))} .
$$

Since each element in $\mathfrak{Q}_{V}^{m}$ is semisimple and simple perverse sheaves are pure, we can extend this definition to entire $\mathfrak{Q}_{V}^{m}$. This induces a bilinear form on $\mathfrak{K}$.

Given any pure complexes $K_{1}, K_{2} \in \mathfrak{Q}_{T}^{m}$ and $L_{1}, L_{2} \in \mathfrak{Q}_{W}^{m}$, we define

$$
\left(K_{1} \otimes L_{1}, K_{2} \otimes L_{2}\right)=t^{2 d}\left(K_{1}, K_{2}\right) \cdot\left(L_{1}, L_{2}\right),
$$

where $d=\sum_{h} \operatorname{dim} T_{h^{\prime}} \operatorname{dim} W_{h^{\prime \prime}}+\sum_{i} \operatorname{dim} T_{i} \operatorname{dim} W_{i}$. Similarly, this can be extended linearly to a bilinear form on $\mathfrak{K} \otimes \mathfrak{K}$.

Proposition 8. Let $K \in \mathfrak{Q}_{T}^{m}, L \in \mathfrak{Q}_{W}^{m}$ and $M \in \mathfrak{Q}_{V}^{m}$ such that $V=T \oplus W$, then

$$
\left(\mathfrak{I n d}_{T, W}^{V}(K \otimes L), M\right)=\left(K \otimes L, \mathfrak{R e} \mathfrak{s}_{T, W}^{V} M\right) .
$$


Proof. Since both $\mathfrak{I n d}$ and $\mathfrak{R e s}$ are additive functors, we can assume that $K, L$ and $M$ are pure complexes. By Lemma 7 in [13], we have

$$
\begin{aligned}
& \left(\mathfrak{I n d}_{T, W}^{V}(K \otimes L), M\right)=\sum_{j \in \mathbb{Z}} d_{j}\left(\mathfrak{I n d}_{T, W}^{V}(K \otimes L), M\right) v^{-j} t^{-\left(\operatorname{wt}\left(\mathfrak{I n d}_{T, W}^{V}(K \otimes L)\right)+\operatorname{wt}(M)\right)} \\
= & \sum_{j \in \mathbb{Z}} d_{j}\left(K \otimes L, \mathfrak{R e} \mathfrak{s}_{T, W}^{V} M\right) v^{-j} t^{-(\operatorname{wt}(K)+\operatorname{wt}(L)+\operatorname{wt}(M))+d} \\
= & \sum_{j \in \mathbb{Z}} d_{j}\left(K \otimes L, \oplus M_{1} \otimes M_{2}\right) v^{-j} t^{-\left(\operatorname{wt}(K)+\operatorname{wt}(L)+\operatorname{wt}\left(M_{1}\right)+\operatorname{wt}\left(M_{2}\right)\right)+2 d}=\sum\left(K, M_{1}\right) \cdot\left(L, M_{2}\right) t^{2 d} .
\end{aligned}
$$

The last equality follows from 8.1.10 (f) in [26]. On the other hand,

$$
\left(K \otimes L, \mathfrak{R e s}_{T, W}^{V} M\right)=\left(K \otimes L, \oplus M_{1} \otimes M_{2}\right)=\sum\left(K, M_{1}\right) \cdot\left(L, M_{2}\right) t^{2 d} .
$$

So $\left(\mathfrak{I n d}_{T, W}^{V}(K \otimes L), M\right)=\left(K \otimes L, \mathfrak{R e s}_{T, W}^{V} M\right)$.

2.12. Fourier-Deligne transformation. Let ' $\Omega$ be a second quiver such that the underlying graph is the same as that of $\Omega$. Denote the source of the arrow $h$ in $\Omega$ by $s(h)=h^{\prime} h$ and its target by $t(h)=" h$. Recall that the source and the target of the arrow $h$ in $\Omega$ are denoted by $s(h)=h^{\prime}$ and $t(h)=h^{\prime \prime}$, respectively. Let $\Omega_{1}=\left\{h \in \Omega \mid{ }^{\prime} h=h^{\prime},{ }^{\prime \prime} h=h^{\prime \prime}\right\}$ and $\Omega_{2}=\left\{h \in \Omega \mid ' h=h^{\prime \prime},{ }^{\prime \prime} h=h^{\prime}\right\}$. For a given $I$-graded $k$-vector space $V$, we denote

$$
\begin{aligned}
& { }^{\prime} E_{V}=\bigoplus_{h \in \Omega_{1}} \operatorname{Hom}\left(V_{h^{\prime}}, V_{h^{\prime \prime}}\right) \oplus \bigoplus_{h \in \Omega_{2}} \operatorname{Hom}\left(V_{h^{\prime \prime}}, V_{h^{\prime}}\right), \\
& \dot{E}_{V}=\bigoplus_{h \in \Omega_{1}} \operatorname{Hom}\left(V_{h^{\prime}}, V_{h^{\prime \prime}}\right) \oplus \bigoplus_{h \in \Omega_{2}} \operatorname{Hom}\left(V_{h^{\prime}}, V_{h^{\prime \prime}}\right) \oplus \bigoplus_{h \in \Omega_{2}} \operatorname{Hom}\left(V_{h^{\prime \prime}}, V_{h^{\prime}}\right) .
\end{aligned}
$$

We have the natural projection maps

$$
E_{V} \stackrel{s}{\longleftarrow} \dot{E}_{V} \stackrel{t}{\longrightarrow}{ }^{\prime} E_{V} .
$$

Recall that to a nontrivial character, $\varphi$, of $\mathbb{F}_{p}$, one can associate a local system $\mathcal{L}_{\varphi}$ on $k$ of rank one. Let $\mathcal{T}_{V}: \dot{E}_{V} \rightarrow k$ be the map defined by

$$
\mathcal{T}_{V}(a, b, c)=\sum_{h \in \Omega_{2}} \operatorname{Tr}\left(V_{h^{\prime}} \stackrel{b}{\rightarrow} V_{h^{\prime \prime}} \stackrel{c}{\rightarrow} V_{h^{\prime}}\right),
$$

where $\operatorname{Tr}$ is the trace function. Denote $\mathcal{L}_{\mathcal{T}_{V}}=\mathcal{T}_{V}^{*} \mathcal{L}_{\varphi}$ which is a rank one $\overline{\mathbb{Q}}_{l}$-local system on $\dot{E}_{V}$. The Fourier-Deligne transformation $\Phi: \mathcal{D}\left(E_{V}\right) \rightarrow \mathcal{D}\left({ }^{\prime} E_{V}\right)$ is defined by

$$
L \mapsto t_{!}\left(s^{*}(L) \otimes \mathcal{L}_{\mathcal{T}_{V}}\left[d_{V}\right]\left(\frac{1}{2} d_{V}\right)\right)
$$

where $d_{V}=\operatorname{dim}\left(\oplus_{h \in \Omega_{2}} \operatorname{Hom}\left(V_{h^{\prime}}, V_{h^{\prime \prime}}\right)\right)$.

For the quiver $\Omega$, one can similarly define ${ }^{\prime} \widetilde{L}_{\underline{\nu}}$, (resp. ' $\mathfrak{L}_{\underline{\nu}}$ and $\mathfrak{Q}_{V}^{m}$ ) as we define $\widetilde{L}_{\underline{\nu}}$, (resp. $\mathfrak{L}_{\underline{\nu}}$ and $\mathfrak{Q}_{V}^{m}$ ) for the quiver $\Omega$.

Proposition 9. (a) The Fourier-Deligne transformation $\Phi$ preserves purity and weight.

(b) $\Phi\left(\widetilde{L}_{\underline{\nu}}\right)=\widetilde{L}_{\underline{\nu}}[M]\left(\frac{M}{2}\right)$, where $M=\sum_{h \in \Omega_{2} ; l^{\prime}<l} \nu_{h^{\prime}}^{l} \nu_{h^{\prime \prime}}^{l^{\prime}}-\nu_{h^{\prime}}^{l^{\prime}} \nu_{h^{\prime \prime}}^{l}$.

(c) $\Phi\left(\mathfrak{L}_{\underline{\nu}}\right)=\mathfrak{L}_{\underline{\nu}}\left(-\frac{M}{2}\right)$. 
Proof. By the definition of $\mathcal{L}_{\mathcal{T}_{V}}$ and Section $2.1(\mathrm{~h})$, we have $\operatorname{wt}\left(\mathcal{L}_{\mathcal{T}_{V}}\right)=0$. Set $\operatorname{wt}(L)=w$. By Section 2.1 (h) again, we have $\operatorname{wt}\left(s^{*}(L) \otimes \mathcal{L}_{\mathcal{T}_{V}}\right)=\operatorname{wt}(L)=w$, i.e., $s^{*}(L) \otimes \mathcal{L}_{\mathcal{T}_{V}} \in$ $\mathcal{D}_{\leq w}\left(\dot{E}_{V}\right) \bigcap \mathcal{D}_{\geq w}\left(\dot{E}_{V}\right)$. So

$$
t_{!}\left(s^{*}(L) \otimes \mathcal{L}_{\mathcal{T}_{V}}\right) \in \mathcal{D}_{\leq w}\left(\dot{E}_{V}\right)
$$

On the other hand, $t_{*}\left(s^{*}(L) \otimes \mathcal{L}_{\mathcal{T}_{V}}\right) \in \mathcal{D}_{\geq w}\left(\dot{E}_{V}\right)$. By [15, 2.1.3],

$$
t_{!}\left(s^{*}(L) \otimes \mathcal{L}_{\mathcal{T}_{V}}\right) \simeq t_{*}\left(s^{*}(L) \otimes \mathcal{L}_{\mathcal{T}_{V}}\right), \quad \forall L \in \mathcal{D}\left(E_{V}\right)
$$

Therefore, we have

$$
t_{!}\left(s^{*}(L) \otimes \mathcal{L}_{\mathcal{T}_{V}}\right) \in \mathcal{D}_{\geq w}\left(\dot{E}_{V}\right) .
$$

By (32) and (33) , we have $\operatorname{wt}\left(t_{!}\left(s^{*}(L) \otimes \mathcal{L}_{\mathcal{T}_{V}}\right)\right)=w=\operatorname{wt}(L)$. Part (a) follows.

By Proposition 10.2.2 in [26], we have $\Phi\left(\widetilde{L}_{\underline{\nu}}\right) \simeq \widetilde{ }^{\prime} \underline{\underline{\nu}}[M]$ up to a Tate twist. So it is enough to check the weights on both sides equal. By Lemma 1 and part (a), wt $\left(\Phi\left(\widetilde{L}_{\underline{\nu}}\right)=\right.$ $\operatorname{wt}\left(\widetilde{L}_{\underline{\nu}}[M]\left(\frac{M}{2}\right)\right)=0$. Part (b) follows.

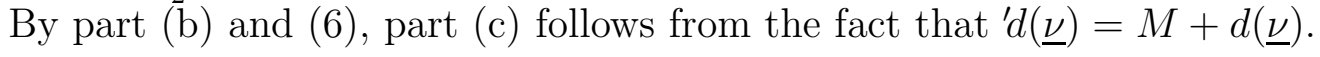

Similarly, one can define a functor $\Phi: \mathcal{D}\left(E_{T} \times E_{W}\right) \rightarrow \mathcal{D}\left({ }^{\prime} E_{T} \times{ }^{\prime} E_{W}\right)$ by replacing $E_{V}$ (resp. ' $\left.E_{V}\right)$ by $E_{T} \times E_{W}\left(\right.$ resp. ' $\left.E_{T} \times{ }^{\prime} E_{W}\right)$.

Proposition 10. For any $K \in \mathfrak{Q}_{T}^{m}$ and $L \in \mathfrak{Q}_{W}^{m}$, we have

$$
\Phi\left(\mathfrak{I n d}_{T, W}^{V}(K \otimes L)\right)=\mathfrak{I n d}_{T, W}^{V}(\Phi(K \otimes L))\left(-d_{0}\right),
$$

where $d_{0}=\frac{1}{2} \sum_{h \in \Omega_{2}}\left(\operatorname{dim} T_{h^{\prime}} \operatorname{dim} W_{h^{\prime \prime}}-\operatorname{dim} W_{h^{\prime}} \operatorname{dim} T_{h^{\prime \prime}}\right)$.

Proof. Since both $\Phi$ and $\mathfrak{I n} \mathfrak{d}_{T, W}^{V}$ are additive functors, we can assume that $K$ and $L$ are pure complexes. By Proposition 10.2.6 in [26], we have $\Phi\left(\mathfrak{I n d} \mathfrak{d}_{T, W}^{V}(K \otimes L)\right) \simeq \mathfrak{I n d}_{T, W}^{V}(\Phi(K \otimes L))$ up to a Tate twist. So it is enough to check that weights on both sides equal. By Proposition 1 and Proposition 9,

$$
\begin{gathered}
\operatorname{wt}\left(\Phi\left(\mathfrak{I n d}_{T, W}^{V}(K \otimes L)\right)\right)=\operatorname{wt}(K)+\operatorname{wt}(L)-\left(d_{1}-d_{2}\right), \text { and } \\
\operatorname{wt}\left(\mathfrak{I n d}_{T, W}^{V}(\Phi(K \otimes L))\left(-d_{0}\right)\right)=\operatorname{wt}(K)+\operatorname{wt}(L)-\left({ }^{\prime} d_{1}-d_{2}\right)+2 d_{0},
\end{gathered}
$$

where ' $d_{1}$ (resp. ' $d_{2}$ ) is defined similarly as $d_{1}$ (resp. $d_{2}$ ) for the new orientation. The proposition follows from the fact that ' $d_{1}-{ }^{\prime} d_{2}-\left(d_{1}-d_{2}\right)=2 d_{0}$.

Proposition 11. For any $K \in \mathfrak{Q}_{T}^{m}$ and $L \in \mathfrak{Q}_{W}^{m}$, we have

$$
\Phi\left(\mathfrak{R e s}_{T, W}^{V}(K)\right)=\mathfrak{R e s}_{T, W}^{V}(\Phi(K))\left(d_{0}\right) .
$$

Proof. This proposition can be proved similarly as Proposition 10.

From Proposition 10, the algebra structure of $\mathfrak{K}$ depends on the orientation of the quiver. We shall show that $\mathfrak{K}$ is independent of the orientation under a twisted multiplication. We define

$$
\widehat{\mathfrak{I n d}}_{T, W}^{V}(K \otimes L)=\mathfrak{I n d}_{T, W}^{V}(K \otimes L)\left(-\frac{d}{2}\right) \text { and } \widehat{\mathfrak{R e s}}_{T, W}^{V}(K)=\mathfrak{R e s}_{T, W}^{V}(K)\left(\frac{d}{2}\right),
$$

where $d$ is the same as the one in Section 2.11, By Propositions 10] and 11, the following proposition holds. 
Proposition 12. For any $K \in \mathfrak{Q}_{T}^{m}$ and $L \in \mathfrak{Q}_{W}^{m}$, we have

$$
\Phi\left(\widehat{\mathfrak{I n d}}_{T, W}^{V}(K \otimes L)\right)=\widehat{\mathfrak{I n d}}_{T, W}^{V}(\Phi(K \otimes L)) \text { and } \Phi\left(\widehat{\mathfrak{R e s}}_{T, W}^{V}(K)\right)=\widehat{\mathfrak{R e s}}_{T, W}^{V}(\Phi(K))
$$

\section{THE ALGEBRA $\mathfrak{f}$}

3.1. The free algebra $\mathfrak{f}$. Recall that $\Omega$ is a matrix satisfying (a),(b),(c) in Section 2.2, In this section, we drop the assumption that $\Omega_{i i}=1$ for any $i \in I$.

For indeterminates $v$ and $t$, we set $v_{i}=v^{i \cdot i / 2}$ and $t_{i}=t^{i \cdot i / 2}$. Moreover, for any rational function $P \in \mathbb{Q}(v, t)$, let $P_{i}$ stand for the rational function obtained from $P$ by substituting $v, t$ by $v_{i}, t_{i}$, respectively. We set $v_{\nu}=\prod_{i} v_{i}^{\nu_{i}}$ and $\operatorname{tr}(\nu)=\sum_{i \in I} \nu_{i} \in \mathbb{Z}$, for any $\nu=\left(\nu_{i}\right)_{i \in I} \in$ $\mathbb{Z}^{I} . t_{\nu}$ is defined similarly.

Let ' be the free unital associative algebra over $\mathbb{Q}(v, t)$ generated by the symbols $\theta_{i}, \forall i \in I$. By setting the degree of the generator $\theta_{i}$ to be $i$, the algebra $f$ becomes an $\mathbb{N}^{I}$-graded algebra. We denote by $\mathfrak{f}_{\nu}$ the subspace of all homogenous elements of degree $\nu$. We have $\mathfrak{f}=\oplus_{\nu \in \mathbb{N}}{ }^{\prime} \mathfrak{f}_{\nu}$, and we denote by $|x|$ the degree of a homogenous element $x \in$ 年.

On the tensor product $\boldsymbol{\uparrow} \otimes \mathfrak{f}$, we define an associative $\mathbb{Q}(v, t)$-algebra structure by

$$
\left(x_{1} \otimes x_{2}\right)\left(y_{1} \otimes y_{2}\right)=v^{-\left|y_{1}\right| \cdot\left|x_{2}\right|} t^{\left\langle\left|y_{1}\right|,\left|x_{2}\right|\right\rangle-\left\langle\left|x_{2}\right|,\left|y_{1}\right|\right\rangle} x_{1} y_{1} \otimes x_{2} y_{2},
$$

for homogeneous elements $x_{1}, x_{2}, y_{1}$ and $y_{2}$ in $\mathfrak{f}$. It is associative since the forms $\langle$,$\rangle in (1)$ and "." in (3) are bilinear.

Similarly, on $\mathfrak{f} \otimes \mathfrak{f} \otimes \mathfrak{f}$, we define an associative $\mathbb{Q}(v, t)$-algebra structure by

$$
\left(x_{1} \otimes x_{2} \otimes x_{3}\right)\left(y_{1} \otimes y_{2} \otimes y_{3}\right)=v^{-M} t^{N} x_{1} y_{1} \otimes x_{2} y_{2} \otimes x_{3} y_{3},
$$

for any homogeneous elements $x_{1}, x_{2}, x_{3}, y_{1}, y_{2}$ and $y_{3}$, where

$$
\begin{gathered}
M=\left|x_{3}\right| \cdot\left|y_{1}\right|+\left|x_{2}\right| \cdot\left|y_{1}\right|+\left|x_{3}\right| \cdot\left|y_{2}\right| \text { and } \\
N=\left\langle\left|y_{1}\right|,\left|x_{3}\right|\right\rangle+\left\langle\left|y_{1}\right|,\left|x_{2}\right|\right\rangle+\left\langle\left|y_{2}\right|,\left|x_{3}\right|\right\rangle-\left\langle\left|x_{3}\right|,\left|y_{1}\right|\right\rangle-\left\langle\left|x_{2}\right|,\left|y_{1}\right|\right\rangle-\left\langle\left|x_{3}\right|,\left|y_{2}\right|\right\rangle .
\end{gathered}
$$

By the equations (35) and (36), one can check that

$$
\begin{aligned}
& \left(x_{1} \otimes x_{2} \otimes x_{3}\right)\left(y_{1} \otimes y_{2} \otimes y_{3}\right) \\
= & v^{-\left|x_{3}\right| \cdot\left(\left|y_{1}\right|+\left|y_{2}\right|\right)} t^{\left\langle\left|y_{1}\right|+\left|y_{2}\right|,\left|x_{3}\right|\right\rangle-\left\langle\left|x_{3}\right|,\left|y_{1}\right|+\left|y_{2}\right|\right\rangle}\left(\left(x_{1} \otimes x_{2}\right)\left(y_{1} \otimes y_{2}\right)\right) \otimes x_{3} y_{3} .
\end{aligned}
$$

Let $r: \mathfrak{f} \rightarrow \mathfrak{f} \otimes$ 角 be the $\mathbb{Q}(v, t)$-algebra homomorphism such that

$$
r\left(\theta_{i}\right)=\theta_{i} \otimes 1+1 \otimes \theta_{i}, \quad \text { for all } i \in I .
$$

Lemma 3. The linear maps $(r \otimes 1) r,(1 \otimes r) r: \mathfrak{f} \rightarrow \mathfrak{f} \otimes \mathfrak{f} \otimes$ 午 are algebra homomorphisms. Moreover, we have the coassociativity property $(r \otimes 1) r=(1 \otimes r) r$.

Proof. By the equation (37) and the bilinearity of $\langle\rangle,, r \otimes 1$ is an algebra homomorphism. Similarly, $1 \otimes r$ is an algebra homomorphism. The first statement follows. The second statement follows from the fact that

$$
(r \otimes 1) r\left(\theta_{i}\right)=\theta_{i} \otimes 1 \otimes 1+1 \otimes \theta_{i} \otimes 1+1 \otimes 1 \otimes \theta_{i}=(1 \otimes r) r\left(\theta_{i}\right),
$$

for all $i \in I$.

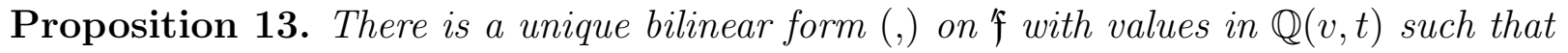

(a) $(1,1)=1$;

(b) $\left(\theta_{i}, \theta_{j}\right)=\delta_{i j} \frac{1}{1-v_{i}^{-2}}$, for all $i, j \in I$; 
(c) $\left(x, y^{\prime} y^{\prime \prime}\right)=\left(r(x), y^{\prime} \otimes y^{\prime \prime}\right), \quad$ for all $x, y^{\prime}, y^{\prime \prime} \in \mathfrak{F}$;

(d) $\left(x^{\prime} x^{\prime \prime}, y\right)=\left(x^{\prime} \otimes x^{\prime \prime}, r(y)\right)$, for all $x^{\prime}, x^{\prime \prime}, y \in$ ' .

Here the bilinear form on $\boldsymbol{\uparrow} \otimes \mathcal{F}$ is defined by

$$
\left(x_{1} \otimes x_{2}, y_{1} \otimes y_{2}\right)=t^{2\left[\left|x_{1}\right|,\left|x_{2}\right|\right]}\left(x_{1}, y_{1}\right)\left(x_{2}, y_{2}\right),
$$

where [,] is defined in (2). Moreover, the bilinear form on $\boldsymbol{\text { ' }}$ is symmetric.

Proof. The proof goes in a similar way as that of Proposition 1.2.3 in [26]. For the convenience of the reader, we present it here. Let $\hat{\uparrow}_{\nu}^{*}$ be the dual space of $\boldsymbol{f}_{\nu}$. We define a bilinear map by

$$
\star: \mathrm{f}_{\nu}^{*} \times \mathrm{f}_{\nu^{\prime}}^{*} \rightarrow \mathrm{f}_{\nu+\nu^{\prime}}^{*}, \quad f, g \mapsto f \star g:=(f \otimes g) r .
$$

By Lemma 3, we have

$$
((f \star g) \star h)=(f \otimes g \otimes h)(r \otimes 1) r=(f \otimes g \otimes h)(1 \otimes r) r=f \star(g \star h)) .
$$

So the bilinear map $\star$ defines an associative algebra structure on $\oplus_{\nu \in \mathbb{N}^{I}} \mathrm{f}_{\nu}^{*}$. Now we define a new multiplication on $\oplus_{\nu}$ 得 $_{\nu}^{*}$ by

$$
\circ: \mathrm{f}_{\nu}^{*} \times \mathrm{f}_{\nu^{\prime}}^{*} \rightarrow \mathrm{f}_{\nu+\nu^{\prime}}^{*}, \quad f, g \mapsto f \circ g:=t^{2\left[\nu, \nu^{\prime}\right]} f \star g .
$$

Since [,] is a bilinear form, $\oplus_{\nu}$ f $_{\nu}^{*}$ equipped with "०" is also an associative algebra. For the rest of the proof, we assume that $\oplus_{\nu}$ f $_{\nu}^{*}$ is the algebra equipped with the multiplication "o".

For any $i \in I$, let $\vartheta_{i} \in$ f $_{i}^{*}$ be the linear map given by $\vartheta_{i}\left(\theta_{i}\right)=\frac{1}{1-v_{i}^{-2}}$. Since f $^{\text {is }}$ a free algebra, there is a unique algebra homomorphism $\zeta:$ 角 $\rightarrow \oplus_{\nu}$ 炡 $^{*}$ such that $\zeta\left(\theta_{i}\right)=\vartheta_{i}$ for all $i \in I$. For any $x, y \in \mathcal{F}$, we set

$$
(x, y)=\zeta(y)(x)
$$

By the definition of $\zeta$, (a) and (b) in the proposition follows automaically. We now show that (c) holds. Since $\zeta$ is an $\mathbb{N}^{I}$-graded algebra homomorphism and preserves the grading, we have

$$
(x, y)=0 \text { if } x, y \text { are homogeneous and }|x| \neq|y| .
$$

We write $r(x)=\sum x_{1} \otimes x_{2}$. By (41), we have the following.

$$
\begin{aligned}
& \left(x, y^{\prime} y^{\prime \prime}\right)=\zeta\left(y^{\prime} y^{\prime \prime}\right)(x)=t^{2\left[\left|y^{\prime}\right|,\left|y^{\prime \prime}\right|\right]}\left(\zeta\left(y^{\prime}\right) \star \zeta\left(y^{\prime \prime}\right)\right)(x)=t^{2\left[\left|y^{\prime}\right|,\left|y^{\prime \prime}\right|\right]}\left(\zeta\left(y^{\prime}\right) \otimes \zeta\left(y^{\prime \prime}\right)\right) r(x) \\
& =t^{2\left[y^{\prime}|,| y^{\prime \prime} \mid\right]} \sum\left(\zeta\left(y^{\prime}\right) \otimes \zeta\left(y^{\prime \prime}\right)\right)\left(x_{1} \otimes x_{2}\right)=t^{2\left[\left|y^{\prime}\right|,\left|y^{\prime \prime}\right|\right]} \sum\left(x_{1}, y^{\prime}\right)\left(x_{2}, y^{\prime \prime}\right)=\left(r(x), y^{\prime} \otimes y^{\prime \prime}\right) .
\end{aligned}
$$

Hence, (c) follows.

Next, we show that (d) holds. Suppose that $y=\theta_{i}$ for some $i \in I$. If $x^{\prime}=\theta_{i}$ and $x^{\prime \prime}=1$ or $x^{\prime}=1$ and $x^{\prime \prime}=\theta_{i}$, we have

$$
\left(x^{\prime} x^{\prime \prime}, y\right)=\left(1-v_{i}^{-2}\right)^{-1}=\left(x^{\prime} \otimes x^{\prime \prime}, r(y)\right) .
$$

By (42), (d) holds for the case $y=\theta_{i}$. Now we assume that (d) holds for $y^{\prime}$ and $y^{\prime \prime}$, we are going to show that (d) holds for $y=y^{\prime} y^{\prime \prime}$. Due to the fact that (,) is a bilinear form, we can assume that $x^{\prime}, x^{\prime \prime}, y^{\prime}, y^{\prime \prime}$ are all homogeneous. Let

$$
r\left(x^{\prime}\right)=\sum x_{1}^{\prime} \otimes x_{2}^{\prime}, \quad r\left(x^{\prime \prime}\right)=\sum x_{1}^{\prime \prime} \otimes x_{2}^{\prime \prime}, \quad r\left(y^{\prime}\right)=\sum y_{1}^{\prime} \otimes y_{2}^{\prime}, \quad r\left(y^{\prime \prime}\right)=\sum y_{1}^{\prime \prime} \otimes y_{2}^{\prime \prime},
$$

such that all factors are homogeneous. Then

$$
r\left(x^{\prime} x^{\prime \prime}\right)=\sum v^{-\left|x_{2}^{\prime}\right| \cdot\left|x_{1}^{\prime \prime}\right|} t^{\left\langle\left|x_{1}^{\prime \prime}\right|,\left|x_{2}^{\prime}\right|\right\rangle-\left\langle\left|x_{2}^{\prime}\right|,\left|x_{1}^{\prime \prime}\right|\right\rangle}\left(x_{1}^{\prime} x_{1}^{\prime \prime} \otimes x_{2}^{\prime} x_{2}^{\prime \prime}\right) \text {, and }
$$


So we have

$$
r\left(y^{\prime} y^{\prime \prime}\right)=\sum v^{-\left|y_{2}^{\prime}\right| \cdot\left|y_{1}^{\prime \prime}\right|} t^{\left\langle\left|y_{1}^{\prime \prime}\right|,\left|y_{2}^{\prime}\right|\right\rangle-\left\langle\left|y_{2}^{\prime}\right|,\left|y_{1}^{\prime \prime}\right|\right\rangle}\left(y_{1}^{\prime} y_{1}^{\prime \prime} \otimes y_{2}^{\prime} y_{2}^{\prime \prime}\right)
$$

$$
\begin{aligned}
& \left(x^{\prime} x^{\prime \prime}, y^{\prime} y^{\prime \prime}\right)=\zeta\left(y^{\prime} y^{\prime \prime}\right)\left(x^{\prime} x^{\prime \prime}\right)=t^{2\left[\left|y^{\prime}\right|,\left|y^{\prime \prime}\right|\right]}\left(\zeta\left(y^{\prime}\right) \otimes \zeta\left(y^{\prime \prime}\right)\right) r\left(x^{\prime} x^{\prime \prime}\right) \\
& =\sum v^{-\left|x_{2}^{\prime}\right| \cdot\left|x_{1}^{\prime \prime}\right|} t^{C_{1}}\left(x_{1}^{\prime} x_{1}^{\prime \prime}, y^{\prime}\right)\left(x_{2}^{\prime} x_{2}^{\prime \prime}, y^{\prime \prime}\right)=\sum v^{-\left|x_{2}^{\prime}\right| \cdot\left|x_{1}^{\prime \prime}\right|} t^{C_{1}}\left(x_{1}^{\prime} \otimes x_{1}^{\prime \prime}, r\left(y^{\prime}\right)\right)\left(x_{2}^{\prime} \otimes x_{2}^{\prime \prime}, r\left(y^{\prime \prime}\right)\right) \\
& =\sum v^{-\left|x_{2}^{\prime}\right| \cdot\left|x_{1}^{\prime \prime}\right|} t^{C}\left(x_{1}^{\prime}, y_{1}^{\prime}\right)\left(x_{1}^{\prime \prime}, y_{2}^{\prime}\right)\left(x_{2}^{\prime}, y_{1}^{\prime \prime}\right)\left(x_{2}^{\prime \prime}, y_{2}^{\prime \prime}\right),
\end{aligned}
$$

where $C_{1}=\left\langle\left|x_{1}^{\prime \prime}\right|,\left|x_{2}^{\prime}\right|\right\rangle-\left\langle\left|x_{2}^{\prime}\right|,\left|x_{1}^{\prime \prime}\right|\right\rangle+2\left[\left|y^{\prime}\right|,\left|y^{\prime \prime}\right|\right]$ and $C=\left\langle\left|x_{1}^{\prime \prime}\right|,\left|x_{2}^{\prime}\right|\right\rangle-\left\langle\left|x_{2}^{\prime}\right|,\left|x_{1}^{\prime \prime}\right|\right\rangle+2\left(\left[\left|y^{\prime}\right|,\left|y^{\prime \prime}\right|\right]+\right.$ $\left.\left[\left|x_{1}^{\prime}\right|,\left|x_{1}^{\prime \prime}\right|\right]+\left[\left|x_{2}^{\prime}\right|,\left|x_{2}^{\prime \prime}\right|\right]\right)$. On the other hand, $\left(x^{\prime} \otimes x^{\prime \prime}, r\left(y^{\prime} y^{\prime \prime}\right)\right)$ is equal to

$$
\begin{aligned}
& \sum v^{-\left|y_{2}^{\prime}\right| \cdot\left|y_{1}^{\prime \prime}\right|} t^{\left\langle\left|y_{1}^{\prime \prime}\right|,\left|y_{2}^{\prime}\right|\right\rangle-\left\langle\left|y_{2}^{\prime}\right|,\left|y_{1}^{\prime \prime}\right|\right\rangle}\left(x^{\prime} \otimes x^{\prime \prime}, y_{1}^{\prime} y_{1}^{\prime \prime} \otimes y_{2}^{\prime} y_{2}^{\prime \prime}\right)=\sum v^{-\left|y_{2}^{\prime}\right| \cdot\left|y_{1}^{\prime \prime}\right|} t^{D_{1}}\left(x^{\prime}, y_{1}^{\prime} y_{1}^{\prime \prime}\right)\left(x^{\prime \prime}, y_{2}^{\prime} y_{2}^{\prime \prime}\right) \\
= & \sum v^{-\left|y_{2}^{\prime}\right| \cdot\left|y_{1}^{\prime \prime}\right|} t^{D_{1}}\left(r\left(x^{\prime}\right), y_{1}^{\prime} \otimes y_{1}^{\prime \prime}\right)\left(r\left(x^{\prime \prime}\right), y_{2}^{\prime} \otimes y_{2}^{\prime \prime}\right)=\sum v^{-\left|y_{2}^{\prime}\right| \cdot\left|y_{1}^{\prime \prime}\right|} t^{D}\left(x_{1}^{\prime}, y_{1}^{\prime}\right)\left(x_{1}^{\prime \prime}, y_{2}^{\prime}\right)\left(x_{2}^{\prime}, y_{1}^{\prime \prime}\right)\left(x_{2}^{\prime \prime}, y_{2}^{\prime \prime}\right),
\end{aligned}
$$

where $D_{1}=\left\langle\left|y_{1}^{\prime \prime}\right|,\left|y_{2}^{\prime}\right|\right\rangle-\left\langle\left|y_{2}^{\prime}\right|,\left|y_{1}^{\prime \prime}\right|\right\rangle+2\left[\left|x^{\prime}\right|,\left|x^{\prime \prime}\right|\right]$ and $D=\left\langle\left|y_{1}^{\prime \prime}\right|,\left|y_{2}^{\prime}\right|\right\rangle-\left\langle\left|y_{2}^{\prime}\right|,\left|y_{1}^{\prime \prime}\right|\right\rangle+2\left(\left[\left|x^{\prime}\right|,\left|x^{\prime \prime}\right|\right]+\right.$ $\left.\left[\left|x_{1}^{\prime}\right|,\left|x_{2}^{\prime}\right|\right]+\left[\left|x_{1}^{\prime \prime}\right|,\left|x_{2}^{\prime \prime}\right|\right]\right)$. By (42) and induction hypothesis, Part (d) is reduced to show that $C=D$ under the following assumption.

$$
\left|x_{1}^{\prime}\right|=\left|y_{1}^{\prime}\right|,\left|x_{2}^{\prime}\right|=\left|y_{1}^{\prime \prime}\right|,\left|x_{1}^{\prime \prime}\right|=\left|y_{2}^{\prime}\right|,\left|x_{2}^{\prime \prime}\right|=\left|y_{2}^{\prime \prime}\right| \text { and }\left|x_{2}^{\prime}\right| \cdot\left|x_{1}^{\prime \prime}\right|=\left|y_{2}^{\prime}\right| \cdot\left|y_{1}^{\prime \prime}\right| .
$$

By the definition of the bilinear forms $\langle$,$\rangle and [$,$] , we have$

$$
\left\langle x_{2}^{\prime}, x_{1}^{\prime \prime}\right\rangle+\left[x_{2}^{\prime}, x_{1}^{\prime \prime}\right]=\left\langle x_{1}^{\prime \prime}, x_{2}^{\prime}\right\rangle+\left[x_{1}^{\prime \prime}, x_{2}^{\prime}\right]
$$

Thus, both $C$ and $D$ are equal to

$2\left[\left|x_{1}^{\prime}\right|,\left|x_{2}^{\prime}\right|\right]+2\left[\left|x_{1}^{\prime}\right|,\left|x_{2}^{\prime \prime}\right|\right]+\left[\left|x_{1}^{\prime \prime}\right|,\left|x_{2}^{\prime}\right|\right]+\left[\left|x_{2}^{\prime}\right|,\left|x_{1}^{\prime \prime}\right|\right]+2\left[\left|x_{1}^{\prime \prime}\right|,\left|x_{2}^{\prime \prime}\right|\right]+2\left[\left|x_{1}^{\prime}\right|,\left|x_{1}^{\prime \prime}\right|\right]+2\left[\left|x_{2}^{\prime}\right|,\left|x_{2}^{\prime \prime}\right|\right]$.

Part (d) follows. Finally, the uniqueness of the bilinear form follows from (b), (c) and (d), and the symmetry of $($,$) follows from the uniqueness of ($,$) .$

3.2. The bialgebra $\mathfrak{f}$. Let $\mathfrak{I}$ be the radical of the bilinear form (,). By an argument exactly the same as that in Section 1.2.3 in [26], we have

Lemma 4. $\mathfrak{I}$ is a two-sided ideal of '午.

Let $\mathfrak{f}=\mathfrak{f} / \mathfrak{I}$ be the quotient algebra of $\mathfrak{f}$ by the ideal $\mathfrak{I}$. By (42), $\mathfrak{I}$ is $\mathbb{N}^{I}$-graded. This implies that $\mathfrak{f}$ is also an $\mathbb{N}^{I}$-graded algebra over $\mathbb{Q}(v, t)$. By abuse of notation, we denote again by $\theta_{i}$ the image of $\theta_{i}$ in $\mathfrak{f}$ under the quotient map. Moreover, the bilinear form $($,$) on$ $\mathfrak{f}$ induces a well-defined symmetric bilinear form, denoted again by $($,$) , since \mathfrak{I}$ is the radical of $($,$) .$

We claim that the bilinear form on $\mathfrak{f}$ is non-degenerate. Assume that it is not, then there exists a nonzero element, say $x$, in $\mathfrak{f}$ such that $(x, y)=0$ for all $y \in \mathfrak{f}$. Let $x^{\prime} \in \mathfrak{f}$ be a representative of $x$, then $\left(x^{\prime}+\mathfrak{I}, z+\mathfrak{I}\right)=0$ for all $z \in \mathfrak{f}$. So $x^{\prime} \in \mathfrak{I}$. A contradiction. The claim follows.

We claim that the radical of the bilinear form on $\mathfrak{f} \otimes$ 争 in Proposition 13 is $\mathfrak{I} \otimes \mathfrak{f}+\mathfrak{f} \otimes \mathfrak{I}$. Assume that $x \otimes y$ is in the radical of the bilinear form on $\mathcal{f} \otimes \mathfrak{f}$, then for any element $x^{\prime} \otimes y^{\prime} \in \mathcal{f} \otimes$ 争, we have

$$
\left(x \otimes y, x^{\prime} \otimes y^{\prime}\right)=t^{2[|x|,|y|]}\left(x, x^{\prime}\right)\left(y, y^{\prime}\right)=0 .
$$


Hence $\left(x, x^{\prime}\right)=0$ or $\left(y, y^{\prime}\right)=0$, i.e., $x \in \mathfrak{I}$ or $y \in \mathfrak{I}$. Thus $x \otimes y \in \mathfrak{I} \otimes \mathfrak{f}+\mathfrak{f} \otimes \mathfrak{I}$. The claim follows.

Moreover, we have

$$
r(\mathfrak{I}) \subset \mathfrak{I} \otimes \mathfrak{f}+\mathfrak{f} \otimes \mathfrak{I} .
$$

Indeed, if $x \in \mathfrak{I}$, then we have $(r(x), y \otimes z)=(x, y z)=0, \quad \forall y, z \in$ 年. This implies that $r(x)$ is in the radical of $($,$) on \boldsymbol{f} \otimes$ 争.

By (44), the map $r$ induces an algebra homomorphism $\mathfrak{f} \rightarrow \mathfrak{f} \otimes \mathfrak{f}$, denoted by $r$ again. Here the algebra structure on $\mathfrak{f} \otimes \mathfrak{f}$ is defined by equation (35). It is clear that $r\left(\theta_{i}\right)=\theta_{i} \otimes 1+1 \otimes \theta_{i}$ for any $\theta_{i} \in \mathfrak{f}$. So the coassociativity property in Lemma 3 still holds for $\mathfrak{f}$.

Let $^{-}: \mathbb{Q}(v, t) \rightarrow \mathbb{Q}(v, t)$ be the unique $\mathbb{Q}(t)$-algebra involution such that

$$
\bar{v}=v^{-1} \text { and } \bar{t}=t .
$$

Let $^{-}:$午 $\rightarrow$ 年 be the unique $\mathbb{Q}(t)$-algebra involution such that

$$
\overline{p \theta_{i}}=\bar{p} \theta_{i} \text { for all } p \in \mathbb{Q}(v, t) \text { and } i \in I \text {. }
$$

From the definition of "-" on 角, we have $|\bar{x}|=|x|$ for any homogeneous element $x \in$ 年.

Lemma 5. If $x \in \mathcal{Y}$ is a homogeneous element and $r(x)=\sum x_{1} \otimes x_{2}$, then we have

$$
r(\bar{x})=\sum v^{-\left|x_{1}\right| \cdot\left|x_{2}\right|} t^{\left\langle\left|x_{2}\right|,\left|x_{1}\right|\right\rangle-\left\langle\left|x_{1}\right|,\left|x_{2}\right|\right\rangle} \bar{x}_{2} \otimes \bar{x}_{1} .
$$

Proof. It is clear that the lemma holds if $x=\theta_{i}$ for any $i \in I$. Assume that the lemma holds for the homogeneous elements $x^{\prime}$ and $x^{\prime \prime}$. We shall show that the lemma holds for $x=x^{\prime} x^{\prime \prime}$. Let us write

$$
r\left(x^{\prime}\right)=\sum x_{1}^{\prime} \otimes x_{2}^{\prime} \quad \text { and } \quad r\left(x^{\prime \prime}\right)=\sum x_{1}^{\prime \prime} \otimes x_{2}^{\prime \prime}
$$

such that all factors are homogeneous. By assumption, we have

$r\left(\overline{x^{\prime}}\right)=\sum v^{-\left|x_{1}^{\prime}\right| \cdot\left|x_{2}^{\prime}\right|} t^{\left\langle\left|x_{2}^{\prime}\right|,\left|x_{1}^{\prime}\right|\right\rangle-\left\langle\left|x_{1}^{\prime}\right|,\left|x_{2}^{\prime}\right|\right\rangle} \overline{x_{2}^{\prime}} \otimes \overline{x_{1}^{\prime}}, \quad r\left(\overline{x^{\prime \prime}}\right)=\sum v^{-\left|x_{1}^{\prime \prime}\right| \cdot\left|x_{2}^{\prime \prime}\right|} t^{\left\langle\left|x_{2}^{\prime \prime}\right|,\left|x_{1}^{\prime \prime}\right|\right\rangle-\left\langle\left|x_{1}^{\prime \prime}\right|,\left|x_{2}^{\prime \prime}\right|\right\rangle} \overline{x_{2}^{\prime \prime}} \otimes \overline{x_{1}^{\prime \prime}}$.

Hence, $r(\bar{x})=r\left(\overline{x^{\prime}}\right) r\left(\overline{x^{\prime \prime}}\right)$ is equal to

$\sum v^{-\left(\left|x_{1}^{\prime}\right|+\left|x_{1}^{\prime \prime}\right|\right) \cdot\left(\left|x_{2}^{\prime}\right|+\left|x_{2}^{\prime \prime}\right|\right)} t^{\left\langle\left|x_{2}^{\prime}\right|+\left|x_{2}^{\prime \prime}\right|,\left|x_{1}^{\prime}\right|+\left|x_{1}^{\prime \prime}\right|\right\rangle-\left\langle\left|x_{1}^{\prime}\right|+\left|x_{1}^{\prime \prime}\right|,\left|x_{2}^{\prime}\right|+\left|x_{2}^{\prime \prime}\right|\right\rangle} v^{\left|x_{1}^{\prime \prime}\right| \cdot\left|x_{2}^{\prime}\right|} t^{\left\langle\left|x_{1}^{\prime \prime}\right|,\left|x_{2}^{\prime}\right|\right\rangle-\left\langle\left|x_{2}^{\prime}\right|,\left|x_{1}^{\prime \prime}\right|\right\rangle} \overline{x_{2}^{\prime} x_{2}^{\prime \prime}} \otimes \overline{x_{1}^{\prime} x_{1}^{\prime \prime}}$.

On the other hand,

$$
r(x)=r\left(x^{\prime} x^{\prime \prime}\right)=\sum v^{-\left|x_{2}^{\prime}\right| \cdot\left|x_{1}^{\prime \prime}\right|} t^{\left\langle\left|x_{1}^{\prime \prime}\right|,\left|x_{2}^{\prime}\right|\right\rangle-\left\langle\left|x_{2}^{\prime}\right|,\left|x_{1}^{\prime \prime}\right|\right\rangle} x_{1}^{\prime} x_{1}^{\prime \prime} \otimes x_{2}^{\prime} x_{2}^{\prime \prime} .
$$

By (45), we have

$$
\overline{v^{-\left|x_{2}^{\prime}\right| \cdot\left|x_{1}^{\prime \prime}\right|} t^{\left\langle\left|x_{1}^{\prime \prime}\right|,\left|x_{2}^{\prime}\right|\right\rangle-\left\langle\left|x_{2}^{\prime}\right|,\left|x_{1}^{\prime \prime}\right|\right\rangle} x_{2}^{\prime} x_{2}^{\prime \prime}}=v^{\left|x_{1}^{\prime \prime}\right| \cdot\left|x_{2}^{\prime}\right|} t^{\left\langle\left|x_{1}^{\prime \prime}\right|, \quad\left|x_{2}^{\prime}\right|\right\rangle-\left\langle\left|x_{2}^{\prime}\right|,\left|x_{1}^{\prime \prime}\right|\right\rangle} \overline{x_{2}^{\prime} x_{2}^{\prime \prime}} .
$$

Since $\left|x_{1}^{\prime} x_{1}^{\prime \prime}\right|=\left|x_{1}^{\prime}\right|+\left|x_{1}^{\prime \prime}\right|$ and $\left|x_{2}^{\prime} x_{2}^{\prime \prime}\right|=\left|x_{2}^{\prime}\right|+\left|x_{2}^{\prime \prime}\right|$, the lemma follows by induction on $\operatorname{tr}(|x|)$.

Lemma 6. The involution map ${ }^{-}:$午 $\rightarrow$ 午 sends $\mathfrak{I}$ onto itself.

Proof. Given any element $x \in \mathfrak{I}$, it is enough to show that $(\bar{x}, y)=0$ for any $y \in \mathfrak{f}$. We can assume that $x$ is a homogenous element since "-" is additive. We shall show that $(\bar{x}, y)=0$ by induction on $\operatorname{tr}(|x|)$. Without lost of generality, we assume that $y$ is a monomial and $y=y^{\prime} y^{\prime \prime}$ for some monomials $y^{\prime}$ and $y^{\prime \prime}$. Since $\theta_{i} \notin \mathfrak{I}$, for any $i \in I$, we have $\operatorname{tr}(|x|) \geq 2$ for 
any element $x \in \mathfrak{I}$. Hence we can further assume that $\operatorname{tr}\left(\left|y^{\prime}\right|\right), \operatorname{tr}\left(\left|y^{\prime \prime}\right|\right)>0$, i.e., $y^{\prime} \notin \mathbb{Q}(v, t)$ and $y^{\prime \prime} \notin \mathbb{Q}(v, t)$.

Write $r(x)=\sum x_{1} \otimes x_{2}$. From (44), $r(x) \in \mathfrak{f} \otimes \mathfrak{I}+\mathfrak{I} \otimes$ 年. Hence either $x_{1} \in \mathfrak{I}$ or $x_{2} \in \mathfrak{I}$. If $x \in \mathfrak{I}$ satisfies that $\operatorname{tr}|x| \leq \operatorname{tr}\left|x^{\prime}\right|$ for all $x^{\prime} \in \mathfrak{I}$, then either $x_{1} \in \mathbb{Q}(v, t)$ or $x_{2} \in \mathbb{Q}(v, t)$, i.e., either $\operatorname{tr}\left(\left|x_{1}\right|\right)=0$ or $\operatorname{tr}\left(\left|x_{2}\right|\right)=0$. By Lemma 5, we have

$$
\left(\bar{x}, y^{\prime} y^{\prime \prime}\right)=\left(r(\bar{x}), y^{\prime} \otimes y^{\prime \prime}\right)=\sum v^{-\left|x_{1}\right| \cdot\left|x_{2}\right|} t^{\left\langle\left|x_{2}\right|,\left|x_{1}\right|\right\rangle-\left\langle\left|x_{1}\right|,\left|x_{2}\right|\right\rangle+2\left[\left|x_{2}\right|,\left|x_{1}\right|\right]}\left(\overline{x_{2}}, y^{\prime}\right)\left(\overline{x_{1}}, y^{\prime \prime}\right)=0 .
$$

This shows that $r(\bar{x}) \in \mathfrak{I}$ if $x \in \mathfrak{I}$ and $\operatorname{tr}|x| \leq \operatorname{tr}\left|x^{\prime}\right|$ for all $x^{\prime} \in \mathfrak{I}$.

We now assume that $\bar{z} \in \mathfrak{I}$ for any $z \in \mathfrak{I}$ such that $\operatorname{tr}(|z|)<\operatorname{tr}(|x|)$. By Lemma 5 again,

$$
\left(\bar{x}, y^{\prime} y^{\prime \prime}\right)=\left(r(\bar{x}), y^{\prime} \otimes y^{\prime \prime}\right)=\sum v^{-\left|x_{1}\right| \cdot\left|x_{2}\right|} t^{\left\langle\left|x_{2}\right|,\left|x_{1}\right|\right\rangle-\left\langle\left|x_{1}\right|,\left|x_{2}\right|\right\rangle+2\left[\left|x_{2}\right|,\left|x_{1}\right|\right]}\left(\overline{x_{2}}, y^{\prime}\right)\left(\overline{x_{1}}, y^{\prime \prime}\right) .
$$

We may assume that both $\operatorname{tr}\left(\left|x_{1}\right|\right)<\operatorname{tr}(|x|)$ and $\operatorname{tr}\left(\left|x_{2}\right|\right)<\operatorname{tr}(|x|)$ by the assumption $\operatorname{tr}\left(\left|y^{\prime}\right|\right)>$ 0 and $\operatorname{tr}\left(\left|y^{\prime \prime}\right|\right)>0$. Therefore, by the induction assumption, $\overline{x_{1}}, \overline{x_{2}} \in \mathfrak{I}$. This implies that $\left(\bar{x}, y^{\prime} y^{\prime \prime}\right)=0$. This finishes the proof.

By Lemma [6, the involution ${ }^{-}:$' $\rightarrow$ 角 induces an involution on $\mathfrak{f}$, denoted by the same notation.

3.3. Quantum Serre relations. For any $i \in I$, let $r_{i}:$ 午 $\rightarrow$ 午 be the unique linear map satisfying the following properties:

$$
r_{i}(1)=0, \quad r_{i}\left(\theta_{j}\right)=\delta_{i j} \quad \forall j \in I, \quad \text { and } \quad r_{i}(x y)=v^{-i \cdot|y|} t^{\langle|y|, i\rangle-\langle i,|y|\rangle} r_{i}(x) y+x r_{i}(y),
$$

for any homogeneous elements $x$ and $y$. If we write $r(x)=\sum x_{1} \otimes x_{2}$ with $x_{1}, x_{2}$ homogenous and $x_{2}$ 's of different degree, then we have

$$
x_{1}=r_{i}(x), \quad \text { if } x_{2}=\theta_{i} .
$$

Since both $r$ and $r_{i}$ are linear maps, it is enough to check this by assuming that $x$ is a monomial. This can be done by induction on $\operatorname{tr}(|x|)$. If $|x|_{i}=0$, then $r_{i}(x)=0$ and there is no term of the form $-\otimes \theta_{i}$ in $r(x)$. The claim holds in this case. Now assume that $|x|_{i} \neq 0$, then we can write $x=x^{\prime} \theta_{i} x^{\prime \prime}$ for some monomials $x^{\prime}, x^{\prime \prime}$ such that $\left|x^{\prime \prime}\right|_{i}=0$. So,

$$
\begin{aligned}
& r_{i}(x)=r_{i}\left(x^{\prime} \theta_{i} x^{\prime \prime}\right)=v^{-i \cdot\left|x^{\prime \prime}\right|} t^{\left\langle\left|x^{\prime \prime}\right|, i\right\rangle-\left\langle i,\left|x^{\prime \prime}\right|\right\rangle} r_{i}\left(x^{\prime} \theta_{i}\right) x^{\prime \prime} \\
= & v^{-i \cdot\left|x^{\prime \prime}\right|-i \cdot i} t^{\left\langle\left|x^{\prime \prime}\right|, i\right\rangle-\left\langle i,\left|x^{\prime \prime}\right|\right\rangle} r_{i}\left(x^{\prime}\right) \theta_{i} x^{\prime \prime}+v^{-i \cdot\left|x^{\prime \prime}\right|} t^{\left\langle\left|x^{\prime \prime}\right|, i\right\rangle-\left\langle i,\left|x^{\prime \prime}\right|\right\rangle} x^{\prime} x^{\prime \prime} .
\end{aligned}
$$

On the other hand,

$$
r(x)=r\left(x^{\prime}\right)\left(\theta_{i} \otimes 1+1 \otimes \theta_{i}\right) r\left(x^{\prime \prime}\right) .
$$

Since $\left|x^{\prime \prime}\right|_{i}=0$, the term $-\otimes \theta_{i}$ only appears in $\left(x^{\prime} \otimes 1\right)\left(1 \otimes \theta_{i}\right)\left(x^{\prime \prime} \otimes 1\right)+\left(z \otimes \theta_{i}\right)\left(\theta_{i} \otimes 1\right)\left(x^{\prime \prime} \otimes 1\right)$ for some $z$. By the induction assumption, $z=r_{i}\left(x^{\prime}\right)$. By (35), the term $-\otimes \theta_{i}$ is

$$
\left(v^{-i \cdot\left|x^{\prime \prime}\right|-i \cdot i} t^{\left\langle\left|x^{\prime \prime}\right|, i\right\rangle-\left\langle i,\left|x^{\prime \prime}\right|\right\rangle} r_{i}\left(x^{\prime}\right) \theta_{i} x^{\prime \prime}+v^{-i \cdot\left|x^{\prime \prime}\right|} t^{\left\langle\left|x^{\prime \prime}\right|, i\right\rangle-\left\langle i,\left|x^{\prime \prime}\right|\right\rangle} x^{\prime} x^{\prime \prime}\right) \otimes \theta_{i} .
$$

The claim follows.

Similarly, for any $i \in I$, there is a unique linear map ${ }_{i} r: \mathcal{f} \rightarrow \mathcal{f}$ satisfying the following properties:

$$
{ }_{i} r(1)=0, \quad{ }_{i} r\left(\theta_{j}\right)=\delta_{i j}, \quad \forall j \in I, \quad \text { and } \quad{ }_{i} r(x y)={ }_{i} r(x) y+v^{-i \cdot|x|} t^{\langle|x|, i\rangle-\langle i,|x|\rangle} x{ }_{i} r(y),
$$

for any homogeneous elements $x, y$. Moreover, we have $r(x)=\theta_{i} \otimes_{i} r(x)$ plus terms of other bihomogeneities. 
Lemma 7. For any $i \in I$, the linear maps ${ }_{i} r, r_{i}: \mathcal{f} \rightarrow$ 午 send $\mathfrak{I}$ to itself.

Proof. For any $x \in \mathfrak{I}$, if $|x|_{i}=0$, then $r_{i}(x)=0 \in \mathfrak{I}$. The lemma holds in this case. We now assume that $|x|_{i} \neq 0$. Write $r(x)=\sum x_{1} \otimes x_{2}$. By (46),$r_{i}(x) \otimes \theta_{i}$ is one of the summands. By (44), either $x_{1} \in \mathfrak{I}$ or $x_{2} \in \mathfrak{I}$. What follows is that $r_{i}(x) \in \mathfrak{I}$ since $\theta_{i} \notin \mathfrak{I}$. It is similar to prove that ${ }_{i} r(x) \in \mathfrak{I}$.

Lemma 8. For any $x, y \in$ 角, we have

$$
\left(y \theta_{i}, x\right)=t^{2[|y|, i]}\left(y, r_{i}(x)\right)\left(\theta_{i}, \theta_{i}\right), \quad\left(\theta_{i} y, x\right)=t^{2[i,|y|]}\left(\theta_{i}, \theta_{i}\right)\left(y,{ }_{i} r(x)\right) .
$$

Proof. By the properties of $r_{i}$, we have

$$
\left(y \theta_{i}, x\right)=\left(y \otimes \theta_{i}, r(x)\right)=\left(y \otimes \theta_{i}, r_{i}(x) \otimes \theta_{i}\right)=t^{2[|y|, i]}\left(y, r_{i}(x)\right)\left(\theta_{i}, \theta_{i}\right),
$$

where the last equality is due to (38). The first one follows. The second one can be proved similarly.

By Lemma 7, ${ }_{i} r$ and $r_{i}$ induce well-defined linear maps on $\mathfrak{f}$, denoted again by the same notations, respectively. Moreover, the property (47) holds in $\mathfrak{f}$.

Lemma 9. Let $x \in \mathfrak{f}_{\nu}$ with $\nu \neq 0$.

(a) If $r_{i}(x)=0$ for all $i \in I$, then $x=0$.

(b) If ${ }_{i} r(x)=0$ for all $i \in I$, then $x=0$.

Proof. If $r_{i}(x)=0$ for all $i \in I$, then, by Lemma 8 , we have $\left(y \theta_{i}, x\right)=0$ for all $y$ and $\theta_{i}$. For any $z \in \mathfrak{f}_{\nu}$ with $\nu \neq 0$, we have $z \in \sum_{i} \mathfrak{f} \theta_{i}$. Therefore $(z, x)=0$ for any $z \in \mathfrak{f}_{\nu}$. This implies $x$ is inside the radical of $($,$) on \mathfrak{f}$. But $($,$) on \mathfrak{f}$ is non-degenerate, so $x=0$. This finishes the proof of (a). (b) can be proved similarly.

For any $n \in \mathbb{N}$, we set

where $v_{i}$ and $t_{i}$ are defined in Section 3.1 ,

$$
\theta_{i}^{(n)}=\frac{\theta_{i}^{n}}{[n]_{v_{i}, t_{i}}^{!}}
$$

Lemma 10. We have $r\left(\theta_{i}^{(n)}\right)=\sum_{p+p^{\prime}=n}\left(v_{i} t_{i}\right)^{-p p^{\prime}} \theta_{i}^{(p)} \otimes \theta_{i}^{\left(p^{\prime}\right)}$, for any $n \in \mathbb{N}$.

Proof. Since $\left(1 \otimes \theta_{i}\right)\left(\theta_{i} \otimes 1\right)=v_{i}^{-2}\left(\theta_{i} \otimes 1\right)\left(1 \otimes \theta_{i}\right)$ and by Section 1.3.5 in [26], we have

$$
r\left(\theta_{i}^{n}\right)=\left(1 \otimes \theta_{i}+\theta_{i} \otimes 1\right)^{n}=\sum_{p+p^{\prime}=n}\left(v_{i} t_{i}\right)^{-p p^{\prime}}\left[\begin{array}{l}
n \\
p
\end{array}\right]_{v_{i}, t_{i}} \theta_{i}^{p} \otimes \theta_{i}^{p^{\prime}} .
$$

The lemma follows from the definitions of $\theta_{i}^{(n)}$ and $\left[\begin{array}{l}n \\ p\end{array}\right]_{v_{i}, t_{i}}$.

For any $n \in \mathbb{N}$ and $i \in I$, by Lemma 10 and (46), we have

$$
r_{i}\left(\theta_{i}^{(n+1)}\right)=\left(v_{i} t_{i}\right)^{-n} \theta_{i}^{(n)} .
$$

Proposition 14. The generators $\theta_{i}$ of $\mathfrak{f}$ satisfy the following identity.

$$
S_{i j}:=\sum_{p+p^{\prime}=1-2 \frac{i \cdot j}{i \cdot i}}(-1)^{p} t_{i}^{-p\left(p^{\prime}-2 \frac{\langle i, j\rangle}{i \cdot i}+2 \frac{\langle j, i\rangle}{i \cdot i}\right)} \theta_{i}^{(p)} \theta_{j} \theta_{i}^{\left(p^{\prime}\right)}=0, \quad \forall i \neq j \in I .
$$


Proof. We set $a^{\prime}=-2 \frac{\langle i, j\rangle}{i \cdot i}, a^{\prime \prime}=-2 \frac{\langle j, i\rangle}{i \cdot i}$ and $N=-2 \frac{i \cdot j}{i \cdot i}$. So $N=a^{\prime}+a^{\prime \prime}$ and we can rewrite $S_{i j}$ as follows:

$$
S_{i j}=\sum_{p+p^{\prime}=N+1}(-1)^{p} t_{i}^{-p\left(p^{\prime}+a^{\prime}-a^{\prime \prime}\right)} \theta_{i}^{(p)} \theta_{j} \theta_{i}^{\left(p^{\prime}\right)} .
$$

By Lemma 9, we only need to show that $r_{k}\left(S_{i j}\right)=0$ for any $k \in I$. It is clear that

$$
r_{k}\left(S_{i j}\right)=0 \quad \text { if } k \neq i, j .
$$

By (48) and the definition of $r_{i}$, we have

$$
\begin{aligned}
& r_{i}\left(\theta_{i}^{(p)} \theta_{j} \theta_{i}^{\left(p^{\prime}\right)}\right)=v^{-2 p^{\prime}\langle i, i\rangle} r_{i}\left(\theta_{i}^{(p)} \theta_{j}\right) \theta_{i}^{\left(p^{\prime}\right)}+\left(v_{i} t_{i}\right)^{1-p^{\prime}} \theta_{i}^{(p)} \theta_{j} \theta_{i}^{\left(p^{\prime}-1\right)} \\
= & v_{i}^{-2 p^{\prime}} v^{-i \cdot j} t^{\langle j, i\rangle-\langle i, j\rangle}\left(v_{i} t_{i}\right)^{1-p} \theta_{i}^{(p-1)} \theta_{j} \theta_{i}^{\left(p^{\prime}\right)}+\left(v_{i} t_{i}\right)^{1-p^{\prime}} \theta_{i}^{(p)} \theta_{j} \theta_{i}^{\left(p^{\prime}-1\right)} \\
= & v_{i}^{-2 p^{\prime}+a^{\prime}+a^{\prime \prime}+1-p} t_{i}^{a^{\prime}+1-p-a^{\prime \prime}} \theta_{i}^{(p-1)} \theta_{j} \theta_{i}^{\left(p^{\prime}\right)}+\left(v_{i} t_{i}\right)^{1-p^{\prime}} \theta_{i}^{(p)} \theta_{j} \theta_{i}^{\left(p^{\prime}-1\right)} .
\end{aligned}
$$

So $r_{i}\left(S_{i j}\right)$ is equal to

$$
\begin{aligned}
& \sum_{1 \leq p \leq N+1}(-1)^{p} t_{i}^{A_{1}} v_{i}^{A_{2}} t_{i}^{A_{3}} \theta_{i}^{(p-1)} \theta_{j} \theta_{i}^{\left(p^{\prime}\right)}+\sum_{0 \leq p \leq N}(-1)^{p} t_{i}^{A_{1}}\left(v_{i} t_{i}\right)^{1-p^{\prime}} \theta_{i}^{(p)} \theta_{j} \theta_{i}^{\left(p^{\prime}-1\right)} \\
= & \sum_{0 \leq p \leq N}(-1)^{p+1} t_{i}^{A_{4}} v_{i}^{A_{2}+1} t_{i}^{A_{3}-1} \theta_{i}^{(p)} \theta_{j} \theta_{i}^{\left(p^{\prime}-1\right)}+\sum_{0 \leq p \leq N}(-1)^{p} t_{i}^{A_{1}}\left(v_{i} t_{i}\right)^{1-p^{\prime}} \theta_{i}^{(p)} \theta_{j} \theta_{i}^{\left(p^{\prime}-1\right)},
\end{aligned}
$$

where $A_{1}=-p\left(p^{\prime}+a^{\prime}-a^{\prime \prime}\right), A_{2}=-2 p^{\prime}+a^{\prime}+a^{\prime \prime}+1-p, A_{3}=a^{\prime}+1-p-a^{\prime \prime}$ and $A_{4}=-(p+1)\left(p^{\prime}-1+a^{\prime}-a^{\prime \prime}\right)$. Since $a^{\prime}+a^{\prime \prime}=N$ and $p+p^{\prime}=N+1$, by comparing the exponents of $v_{i}$ and $t_{i}$ in (50), we have

$$
r_{i}\left(S_{i j}\right)=0 .
$$

By (48) and the definition of $r_{j}$ again, we have

$$
r_{j}\left(\theta_{i}^{(p)} \theta_{j} \theta_{i}^{\left(p^{\prime}\right)}\right)=v^{-p^{\prime} j \cdot i} t^{p^{\prime}(\langle i, j\rangle-\langle j, i\rangle)} \theta_{i}^{(p)} \theta_{i}^{\left(p^{\prime}\right)}=v_{i}^{p^{\prime}\left(a^{\prime}+a^{\prime \prime}\right)} t_{i}^{p^{\prime}\left(a^{\prime \prime}-a^{\prime}\right)} \theta_{i}^{(p)} \theta_{i}^{\left(p^{\prime}\right)} .
$$

So $r_{j}\left(S_{i j}\right)$ is equal to

$$
\begin{aligned}
& \sum_{p+p^{\prime}=N+1}(-1)^{p} t_{i}^{-p\left(p^{\prime}+a^{\prime}-a^{\prime \prime}\right)} v_{i}^{p^{\prime}\left(a^{\prime}+a^{\prime \prime}\right)} t_{i}^{p^{\prime}\left(a^{\prime \prime}-a^{\prime}\right)} \theta_{i}^{(p)} \theta_{i}^{\left(p^{\prime}\right)} \\
= & \sum_{p+p^{\prime}=N+1}(-1)^{p} t_{i}^{-p\left(p^{\prime}+a^{\prime}-a^{\prime \prime}\right)} v_{i}^{p^{\prime}\left(a^{\prime}+a^{\prime \prime}\right)} t_{i}^{p^{\prime}\left(a^{\prime \prime}-a^{\prime}\right)}\left[\begin{array}{c}
N+1 \\
p
\end{array}\right]_{v, t} \theta_{i}^{(N+1)} .
\end{aligned}
$$

By (11), to show that $r_{j}\left(S_{i j}\right)=0$, it is enough to show that

$$
\sum_{p+p^{\prime}=N+1}(-1)^{p} t_{i}^{-p\left(p^{\prime}+a^{\prime}-a^{\prime \prime}\right)} v_{i}^{p^{\prime}\left(a^{\prime}+a^{\prime \prime}\right)} t_{i}^{p^{\prime}\left(a^{\prime \prime}-a^{\prime}\right)+p p^{\prime}}\left[\begin{array}{c}
N+1 \\
p
\end{array}\right]_{v}=0 .
$$

By using $a^{\prime}+a^{\prime \prime}=N$ and $p+p^{\prime}=N+1$, the left hand side of (52) is

$$
t_{i}^{(N+1)\left(a^{\prime \prime}-a^{\prime}\right)} \sum_{p+p^{\prime}=N+1}(-1)^{p} v_{i}^{p\left(a^{\prime}+a^{\prime \prime}\right)}\left[\begin{array}{c}
N+1 \\
p
\end{array}\right]_{v} .
$$

By Section 1.3.4 in [26], this is 0. So we have

$$
r_{j}\left(S_{i j}\right)=0 .
$$

By (49), (51), (53), we have $r_{k}\left(S_{i j}\right)=0, \forall k \in I$. This finishes the proof. 
In Section 4.1, we shall show that the ideal $\mathfrak{J}$ is generated by $\left\{S_{i j}, i \neq j\right\}$.

3.4. Comparison. In this section, we compare the algebra $\mathfrak{f}$ with various versions of quantum algebras in literature.

(a). Two-parameter quantum algebras are defined case by case in [2] and [16]. If we set $v=\left(r s^{-1}\right)^{\frac{1}{2}}$ and $t=(r s)^{-\frac{1}{2}}$, then the quantum Serre relation for $\mathfrak{f}$ coincides with the one in [16] and [2].

(b). Given a Dynkin quiver, Reineke defines a $\mathbb{Q}(\alpha, \beta)$-algebra $\mathcal{H}_{\alpha, \beta}$ in [27]. By Proposition 6.3 in [27], $\mathcal{H}_{\alpha, \beta}$ is isomorphic to the positive part of the two-parameter quantum algebra in [16] associated to the Dynkin quiver. Let $\Omega=I d-A, v=(\alpha \beta)^{\frac{1}{2}}$ and $t=\left(\alpha \beta^{-1}\right)^{\frac{1}{2}}$, where $I d$ is the identity matrix and $A$ is the adjacent matrix of the Dynkin quiver. Then $\mathcal{H}_{\alpha, \beta}$ is isomorphic to $\mathfrak{f}$.

Theorem 7 in Section 5.3 shows that $\mathfrak{K}$ is a geometrization of $\mathfrak{A} \mathfrak{f}$. By the sheaf-function correspondence, we obtain a Hall-algebra construction of $\mathfrak{f}$ associated to arbitrary $\Omega$, not just the one associated to a Dynkin quiver. In a sense, this generalizes Reineke's construction.

(c). In [17, $\mathrm{Hu}$, Pei and Rosso define quantum algebras with multi-parameters $q_{i j}$ associated to $(I, \cdot)$. Let us recall the multi-parameter quantum Serre relations from [17]. It is

$$
\sum_{p+p^{\prime}=1-i \cdot j}(-1)^{p} q_{i i}^{-\frac{p i \cdot j}{2}} q_{j i}^{p}\left[\begin{array}{c}
1-i \cdot j \\
p
\end{array}\right]_{q_{i i}^{\frac{1}{2}}} \theta_{i}^{p} \theta_{j} \theta_{i}^{p^{\prime}}=0 .
$$

Since we use different Gaussian binomial coefficients, (154) is slightly different from the original one in [17]. On the other hand, we can rewrite the quantum Serre relations in Proposition 14 as follows.

$$
\sum_{p+p^{\prime}=1-i \cdot j}(-1)^{p} t^{p(\langle i, j\rangle-\langle j, i\rangle)}\left[\begin{array}{c}
1-i \cdot j \\
p
\end{array}\right]_{v_{i}} \theta_{i}^{p} \theta_{j} \theta_{i}^{p^{\prime}}=0 .
$$

By setting $q_{i i}=v_{i}^{2}$ and $q_{j i}=v^{i \cdot j} t^{\langle i, j\rangle-\langle j, i\rangle}$, (54) is reduced to (55). In other words, $\mathfrak{f}$ is a specialization of a multi-parameter quantum algebra defined in [17].

\section{Specialization AND DEFORMATion}

4.1. Negative part. Recall that $(I, \cdot)$ is the Cartan datum associated to the matrix $\Omega$. If we set $t=1$, the construction in Section 3 is exactly Lusztig's construction in [26]. In particular, the specialization of the bialgebra $(\mathfrak{f}, \cdot r)$ in Section 3.2 at $t=1$ is Lusztig's algebra in [26] associated to $(I, \cdot)$, which we shall denote by $\left(\mathbf{f}, \circ, \widetilde{r}_{1}\right)$, where $\circ$ and $\widetilde{r}_{1}$ are multiplication and comultiplication of $\mathbf{f}$, respectively.

Besides specialization, there is another way to relate these two bialgebras. Namely, we shall show that $\left(\mathbf{f}, \circ, \widetilde{r}_{1}\right)$ can be deformed to $\mathfrak{f}$. Let $\mathbf{f}_{v, t}=\mathbf{f} \otimes_{\mathbb{Q}(v)} \mathbb{Q}(v, t)$. The bialgebra structure on $\mathbf{f}$ can be naturally extended to $\mathbf{f}_{v, t}$, denoted again by $\left(\circ, \widetilde{r}_{1}\right)$.

We define a new multiplication " $\odot$ " on $\mathbf{f}_{v, t}$ by

$$
x \odot y=t^{[|x|,|y|]} x \circ y,
$$

for any homogenous elements $x, y \in \mathbf{f}_{v, t}$, where [,] is defined in (2).

Define a new multiplication, denote again by $\odot$, on $\mathbf{f}_{v, t} \otimes \mathbf{f}_{v, t}$ as follows.

$$
\left(x_{1} \otimes x_{2}\right) \odot\left(y_{1} \otimes y_{2}\right)=v^{-\left|y_{1}\right| \cdot\left|x_{2}\right|} t^{\left\langle\left|y_{1}\right|,\left|x_{2}\right|\right\rangle-\left\langle\left|x_{2}\right|,\left|y_{1}\right|\right\rangle}\left(x_{1} \odot y_{1}\right) \otimes\left(x_{2} \odot y_{2}\right),
$$


for any homogeneous elements $x_{1}, x_{2}, y_{1}$ and $y_{2}$.

For any $x \in \mathbf{f}_{v, t}$, we write $\widetilde{r}_{1}(x)=\sum x_{1} \otimes x_{2}$ with $x_{1}, x_{2}$ homogenous. Define a linear map $r_{1}: \mathbf{f}_{v, t} \rightarrow \mathbf{f}_{v, t} \otimes \mathbf{f}_{v, t}$ by

$$
r_{1}(x)=\sum t^{-\left[\left|x_{1}\right|,\left|x_{2}\right|\right]} x_{1} \otimes x_{2}
$$

Proposition 15. The linear map $r_{1}:\left(\mathbf{f}_{v, t}, \odot\right) \rightarrow\left(\mathbf{f}_{v, t} \otimes \mathbf{f}_{v, t}, \odot\right)$ is an algebra homomorphism.

Proof. For any homogenous elements $x$ and $y$ in $\mathbf{f}_{v, t}$, we write $\widetilde{r}_{1}(x)=\sum x_{1} \otimes x_{2}$ and $\widetilde{r}_{1}(y)=\sum y_{1} \otimes y_{2}$ with $x_{1}, x_{2}, y_{1}$ and $y_{2}$ are all homogenous. Then $\widetilde{r}_{1}(x \circ y)=\sum v^{-\left|x_{2}\right| \cdot\left|y_{1}\right|}\left(x_{1} \circ\right.$ $\left.y_{1}\right) \otimes\left(x_{2} \circ y_{2}\right)$. Therefore,

$$
r_{1}(x \odot y)=t^{[|x|,|y|]} r_{1}(x \circ y)=\sum v^{-\left|x_{2}\right| \cdot\left|y_{1}\right|} t^{[|x|,|y|]} t^{-\left[\left|x_{1}\right|+\left|y_{1}\right|,\left|x_{2}\right|+\left|y_{2}\right|\right]}\left(x_{1} \circ y_{1}\right) \otimes\left(x_{2} \circ y_{2}\right) .
$$

On the other hand, we have

$$
\begin{aligned}
& r_{1}(x) \odot r_{1}(y)=\sum t^{-\left[\left|x_{1}\right|,\left|x_{2}\right|\right]-\left[\left|y_{1}\right|,\left|y_{2}\right|\right]}\left(x_{1} \otimes x_{2}\right) \odot\left(y_{1} \otimes y_{2}\right) \\
= & \sum t^{-\left[\left|x_{1}\right|,\left|x_{2}\right|\right]-\left[\left|y_{1}\right|,\left|y_{2}\right|\right]} v^{-\left|x_{2}\right| \cdot\left|y_{1}\right|} t^{\left\langle\left|y_{1}\right|,\left|x_{2}\right|\right\rangle-\left\langle\left|x_{2}\right|,\left|y_{1}\right|\right\rangle}\left(x_{1} \otimes x_{2}\right) \odot\left(y_{1} \otimes y_{2}\right) .
\end{aligned}
$$

By comparing (59) with (59), it is reduced to check that

$$
[|x|,|y|]-\left[\left|x_{1}\right|+\left|y_{1}\right|,\left|x_{2}\right|+\left|y_{2}\right|\right]=\left\langle\left|y_{1}\right|,\left|x_{2}\right|\right\rangle-\left\langle\left|x_{2}\right|,\left|y_{1}\right|\right\rangle-\left[\left|x_{1}\right|,\left|x_{2}\right|\right]-\left[\left|y_{1}\right|,\left|y_{2}\right|\right],
$$

which follows from (43).

Theorem 3. The assignment $\theta_{i} \mapsto \theta_{i}, \forall i \in I$ gives a twisted bialgebra isomorphism $\mathfrak{f} \simeq$ $\left(\mathbf{f}_{v, t}, \odot, r_{1}\right)$.

Proof. Recall that $\mathcal{f}$ is the free algebra generated by $\theta_{i}, i \in I$. Let ' $\phi: \mathcal{f} \rightarrow \mathbf{f}_{v, t}$ be the algebra homomorphism sending $\theta_{i}$ to $\theta_{i}$, where the algebra structure of $\mathbf{f}_{v, t}$ is defined in (56). Consider the following diagram.

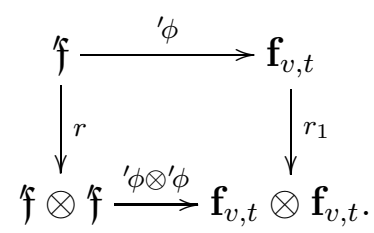

Since all maps are algebra homomorphisms, the diagram commutes by checking the image on $\theta_{i}, \forall i \in I$.

Recall that there is a unique non-degenerate bilinear form, $(,)_{L}$, on $\mathbf{f}$ defined in Chapter 1 in [26] satisfying the following properties.

(a) $(1,1)_{L}=1, \quad\left(\theta_{i}, \theta_{j}\right)_{L}=\delta_{i j} \frac{1}{1-v_{i}^{-2}} \quad$ for all $i, j \in I$,

(b) $\left(x, y^{\prime} \circ y^{\prime \prime}\right)_{L}=\left(\widetilde{r}_{1}(x), y^{\prime} \otimes y^{\prime \prime}\right)_{L} \quad$ for all $x, y^{\prime}, y^{\prime \prime} \in \mathbf{f}$,

(c) $\left(x^{\prime} \circ x^{\prime \prime}, y\right)_{L}=\left(x^{\prime} \otimes x^{\prime \prime}, \widetilde{r}_{1}(y)\right)_{L}$ for all $x^{\prime}, x^{\prime \prime}, y \in \mathbf{f}$.

Since any element $x \in \mathbf{f}_{v, t}$ can be written into $a=\sum a_{i} \otimes t^{i}$, we can extend the bilinear form to $\mathbf{f}_{v, t}$ by setting $\left(x \otimes t^{m}, y \otimes t^{n}\right)_{L}=t^{m+n}(x, y)_{L}$. Moreover, this bilinear form on $\mathbf{f}_{v, t}$ still satisfies the above properties (a), (b), (c).

Now we define a new bilinear form, $(,)^{\prime}$, on $\boldsymbol{f}^{\mathrm{f}}$ as follows.

$$
(x, y)^{\prime}=\left({ }^{\prime} \phi(x),{ }^{\prime} \phi(y)\right)_{L}, \forall x, y \in \text { '列. }
$$


We claim that $\operatorname{Rad}(,)^{\prime}=\operatorname{Ker}\left({ }^{\prime} \phi\right)$, where $\operatorname{Rad}(,)^{\prime}$ is the radical of $(,)^{\prime}$. It is clear that

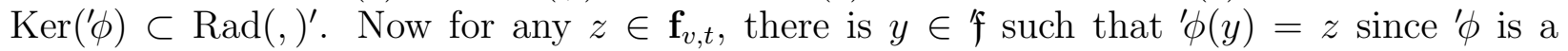
surjective map. Therefore, for any $x \in \operatorname{Rad}(,)^{\prime}, 0=(x, y)^{\prime}=\left({ }^{\prime} \phi(x),{ }^{\prime} \phi(y)\right)_{L}=\left({ }^{\prime} \phi(x), z\right)_{L}$. Since $(,)_{L}$ is non-degenerate and $z$ is arbitrary, ' $\phi(x)=0$. So $x \in \operatorname{Ker}\left({ }^{\prime} \phi\right)$.

To prove that $\mathfrak{f} \simeq\left(\mathbf{f}_{v, t}, \odot\right)$, it is enough to show that $(,)^{\prime}$ satisfying the properties (a), (b), (c),(d) in Proposition 13. The bilinear form on $\mathfrak{f} \otimes$ 午 is defined by

$$
\left(x_{1} \otimes x_{2}, y_{1} \otimes y_{2}\right)^{\prime}=t^{2\left[\left|x_{1}\right|,\left|x_{2}\right|\right]}\left(x_{1}, y_{1}\right)^{\prime}\left(x_{2}, y_{2}\right)^{\prime} .
$$

Properties (a) and (b) are obvious. We now check property (d). For $y \in \mathfrak{f}$, we write $r(y)=\sum y^{\prime} \otimes y^{\prime \prime}$ and $\widetilde{r}_{1}\left({ }^{\prime} \phi(y)\right)=\sum y_{1} \otimes y_{2}$. Then for any $a, b \in$ 午, we have

$$
\begin{gathered}
(a b, y)^{\prime}=\left({ }^{\prime} \phi(a b),{ }^{\prime} \phi(y)\right)_{L}=\left({ }^{\prime} \phi(a) \odot{ }^{\prime} \phi(b),,^{\prime} \phi(y)\right)_{L}=t^{[|a|,|b|]}\left({ }^{\prime} \phi(a) \circ^{\prime} \phi(b),{ }^{\prime} \phi(y)\right)_{L} \\
=t^{[|a|,|b|]}\left({ }^{\prime} \phi(a) \otimes{ }^{\prime} \phi(b), \widetilde{r}_{1}\left({ }^{\prime} \phi(y)\right)\right)_{L}=t^{[|a|,|b|]} \sum\left({ }^{\prime} \phi(a) \otimes{ }^{\prime} \phi(b), y_{1} \otimes y_{2}\right)_{L} .
\end{gathered}
$$

On the other hand, we have

$$
\begin{aligned}
(a & \otimes b, r(y))^{\prime}=\sum\left(a \otimes b, y^{\prime} \otimes y^{\prime \prime}\right)^{\prime}=\sum t^{2[|a|,|b|]}\left(a, y^{\prime}\right)^{\prime}\left(b, y^{\prime \prime}\right)^{\prime} \\
& =\sum t^{2[|a|,|b|]}\left({ }^{\prime} \phi(a),{ }^{\prime} \phi\left(y^{\prime}\right)\right)_{L}\left({ }^{\prime} \phi(b),{ }^{\prime} \phi\left(y^{\prime \prime}\right)\right)_{L}=\sum t^{2[|a|,|b|]}\left({ }^{\prime} \phi(a) \otimes{ }^{\prime} \phi(b),{ }^{\prime} \phi\left(y^{\prime}\right) \otimes^{\prime} \phi\left(y^{\prime \prime}\right)\right)_{L} \\
& =t^{2[|a|,|b|]}\left({ }^{\prime} \phi(a) \otimes{ }^{\prime} \phi(b),\left({ }^{\prime} \phi \otimes \otimes^{\prime} \phi\right)(r(y))\right)_{L}=t^{2[|a|,|b|]}\left({ }^{\prime} \phi(a) \otimes{ }^{\prime} \phi(b), r_{1}\left({ }^{\prime} \phi(y)\right)\right)_{L} \\
& =t^{2[|a|,|b|]}\left({ }^{\prime} \phi(a) \otimes{ }^{\prime} \phi(b), \sum t^{-\left[\left|y_{1}\right|,\left|y_{2}\right|\right]} y_{1} \otimes y_{2}\right)_{L}=t^{[|a|,|b|]} \sum\left({ }^{\prime} \phi(a) \otimes{ }^{\prime} \phi(b), y_{1} \otimes y_{2}\right)_{L} .
\end{aligned}
$$

The last equality follows from the fact that $\left|y_{1}\right|=|a|$ and $\left|y_{2}\right|=|b|$. This proves that the property (d) and (c) can be proved similarly. The claim that $\operatorname{Rad}(,)^{\prime}=\operatorname{Ker}\left({ }^{\prime} \phi\right)$ holds.

The coalgebra homomorphism follows from the commutativity of the Diagram (60). This finishes the proof.

On $\mathbf{f}_{v, t}$, we have two different bialgebra structures, $\left(\mathbf{f}_{v, t}, \circ, \widetilde{r}_{1}\right)$ and $\left(\mathbf{f}_{v, t}, \odot, r_{1}\right)$. By Theorem 3, we have $\mathfrak{f} \simeq\left(\mathbf{f}_{v, t}, \odot, r_{1}\right)$. We now define a second bialgebra structure on $\mathfrak{f}$ corresponding to $\left(\mathbf{f}_{v, t}, \circ, \widetilde{r}_{1}\right)$. Define a new multiplication " $*$ " on $\mathfrak{f}$ by

$$
x * y=t^{-[|x|,|y|]} x y,
$$

for any homogeneous elements $x$ and $y .(\mathfrak{f}, *)$ is an associative algebra due to the fact that $[$,$] is a bilinear form. Define a new multiplication, denote again by *$, on $\mathfrak{f} \otimes \mathfrak{f}$ as follows.

$$
\left(x_{1} \otimes x_{2}\right) *\left(y_{1} \otimes y_{2}\right)=v^{-\left|y_{1}\right| \cdot\left|x_{2}\right|} x_{1} * y_{1} \otimes x_{2} * y_{2},
$$

for any homogeneous elements $x_{1}, x_{2}, y_{1}$ and $y_{2}$. For any $x \in \mathfrak{f}$, we write $r(x)=\sum x_{1} \otimes x_{2}$ with $x_{1}, x_{2}$ homogenous. Define a linear map $\widetilde{r}: \mathfrak{f} \rightarrow \mathfrak{f} \otimes \mathfrak{f}$ by

$$
\widetilde{r}(x)=\sum t^{\left[\left|x_{1}\right|,\left|x_{2}\right|\right]} x_{1} \otimes x_{2} .
$$

By a similar argument as that of Proposition 15, we have that $\widetilde{r}: \mathfrak{f} \rightarrow \mathfrak{f} \otimes \mathfrak{f}$ is an algebra homomorphism with respect to the multiplications $*$ on both sides. By Theorem 3 , we have

Proposition 16. The assignment $\theta_{i} \mapsto \theta_{i}, \forall i \in I$ gives a twisted bialgebra isomorphism $(\mathfrak{f}, *, \widetilde{r}) \simeq\left(\mathbf{f}_{v, t}, \circ, \widetilde{r}_{1}\right)$.

Recall that $\mathfrak{f}=' \mathfrak{f} / \mathfrak{J}$ and $\mathfrak{J}$ is the radical of the bilinear form of $($,$) on \mathfrak{f}$. By Proposition 16, we have 
Corollary 2. $\mathfrak{J}$ is generated by $S_{i j}, \forall i \neq j \in I$, where $S_{i j}$ is defined in Proposition 14.

Suppose $\Omega^{\prime}$ is another matrix satisfying (a),(b),(c) in Section 2.2. Let $\mathfrak{f}\left(\Omega^{\prime}\right)$ be the bialgebra constructed in Section 3.2 associated to $\Omega^{\prime}$. By Proposition [16, we have

Corollary 3. If the associated Cartan datums of $\Omega$ and $\Omega^{\prime}$ are the same, then the assignment $\theta_{i} \mapsto \theta_{i}, \forall i \in I$ gives a twisted bialgebra isomorphism $(\mathfrak{f}, *, \widetilde{r}) \simeq\left(\mathfrak{f}\left(\Omega^{\prime}\right), *, \widetilde{r}\right)$.

Let $\phi: \mathfrak{f} \rightarrow\left(\mathbf{f}_{v, t}, \odot\right)$ be the induced map from ' $\phi:$ ' $\rightarrow\left(\mathbf{f}_{v, t}, \odot\right)$. This is an algebra isomorphism by Theorem 3 . Moreover, one can easily check that

$$
\phi(a * b)=\phi(a) \circ \phi(b), \quad \forall a, b \in \mathfrak{f} .
$$

We define a new bilinear form, denoted by $(,)^{*}$, on $\mathfrak{f}$ and $\mathfrak{f} \otimes \mathfrak{f}$ by

$(66)(a \otimes x, b \otimes y)^{*}=(\phi(a) \otimes \phi(x), \phi(b) \otimes \phi(y))_{L}$, and $(a, b)^{*}=(\phi(a), \phi(b))_{L}, \forall a, b, x, y \in \mathfrak{f}$.

We notice that $($,$) and (,)^{*}$ are different on $\mathfrak{f} \otimes \mathfrak{f}$ by comparing (61) with (66). Moreover, if we consider $\mathbf{f} \otimes 1$ as a subalgebra of $(\mathfrak{f}, *)$ via the map $\phi$, then the restriction of $(,)^{*}$ to $\mathbf{f} \otimes 1$ coincides with the bilinear form $(,)_{L}$ on $\mathbf{f}$ in the proof of Theorem 3 . By (65), (66) and the property of $(,)_{L}$, we have

$$
(a * b, z)^{*}=(a \otimes b, \widetilde{r}(z))^{*}, \quad \forall a, b, z \in \mathfrak{f} .
$$

Proposition 17. For any $x, y \in \mathbf{f} \otimes 1$, we have $(x, y)=(x, y)^{*}$.

Proof. We show it by induction on $\operatorname{tr}(|x|)$. Since both $($,$) and (,)^{*}$ are bilinear on $\mathbf{f} \otimes 1$, we can assume that both $x$ and $y$ are monomials. If $\operatorname{tr}(|x|)=1$, then $x=\theta_{i}$ for some $i \in I$ and $\left(\theta_{i}, \theta_{i}\right)=\left(\theta_{i}, \theta_{i}\right)^{*}$.

If $x \in \mathbf{f} \otimes 1$, by Theorem 3 , $x * \theta_{i}=t^{-[|x|, i]} x \theta_{i} \in \mathbf{f} \otimes 1$. We now assume that $(x, y)=(x, y)^{*}$ for any $y \in \mathbf{f} \otimes 1$ with $|y|=|x|$. We want to show that $\left(x * \theta_{i}, z\right)=\left(x * \theta_{i}, z\right)^{*}$ for any $i \in I$ and any $z \in \mathbf{f} \otimes 1$ with $|z|=|x|+i$. By Lemma 8 , we have

$$
\left(x * \theta_{i}, z\right)=t^{-[|x|, i]}\left(x \theta_{i}, z\right)=t^{[|x|, i]}\left(x, r_{i}(z)\right)\left(\theta_{i}, \theta_{i}\right)
$$

On the other hand, by (46) and (뗘), we have $\widetilde{r}(x)=t^{[|x|, i]} r_{i}(x) \otimes \theta_{i}$ modulo homogeneities terms of different degree at the second component. Therefore, by (67), we have

$$
\left(x * \theta_{i}, z\right)^{*}=\left(x \otimes \theta_{i}, \widetilde{r}(z)\right)^{*}=t^{[|x|, i]}\left(x, r_{i}(z)\right)^{*}\left(\theta_{i}, \theta_{i}\right)^{*}
$$

By the induction assumption, (68) and (69) are equal. Proposition follows.

4.2. Entire algebra. Recall that $\Omega=\left(\Omega_{i j}\right)_{i, j \in I}$ is the matrix fixed in Section 2.2. The two-parameter quantum algebra $U_{v, t}$ associated to $\Omega$ is an associative $\mathbb{Q}(v, t)$-algebra with 1 
generated by symbols $E_{i}, F_{i}, K_{i}^{ \pm 1}, K_{i}^{ \pm 1}, \forall i \in I$ and subject to the following relations.

$$
\begin{aligned}
& K_{i}^{ \pm 1} K_{j}^{ \pm 1}=K_{j}^{ \pm 1} K_{i}^{ \pm 1}, \quad K_{i}^{ \pm 1} K_{j}^{\prime \pm 1}=K_{j}^{ \pm 1} K_{i}^{\prime \pm 1}, \\
& K_{i}^{ \pm 1} K_{j}^{\prime \pm 1}=K_{j}^{ \pm 1} K_{i}^{ \pm 1}, \quad K_{i}^{ \pm 1} K_{i}^{\mp 1}=1=K_{i}^{\prime \pm 1} K_{i}^{\prime \mp 1} . \\
& K_{i} E_{j} K_{i}^{-1}=v^{i \cdot j} t^{\langle i, j\rangle-\langle j, i\rangle} E_{j}, \quad K_{i}^{\prime} E_{j} K_{i}^{\prime-1}=v^{-i \cdot j} t^{\langle i, j\rangle-\langle j, i\rangle} E_{j}, \\
& K_{i} F_{j} K_{i}^{-1}=v^{-i \cdot j} t^{\langle j, i\rangle-\langle i, j\rangle} F_{j}, \quad K_{i}^{\prime} F_{j} K_{i}^{\prime-1}=v^{i \cdot j} t^{\langle j, i\rangle-\langle i, j\rangle} F_{j} . \\
& E_{i} F_{j}-F_{j} E_{i}=\delta_{i j} \frac{K_{i}-K_{i}^{\prime}}{v_{i}-v_{i}^{-1} .} \\
& \quad \sum_{p+p^{\prime}=1-2 \frac{i \cdot j}{i \cdot i}}(-1)^{p} t_{i}^{-p\left(p^{\prime}-2 \frac{\langle i, j\rangle}{i \cdot i}+2 \frac{\langle j, i\rangle}{i \cdot i}\right)} E_{i}^{\left(p^{\prime}\right)} E_{j} E_{i}^{(p)}=0, \quad \text { if } i \neq j, \\
& \quad \sum_{p+p^{\prime}=1-2 \frac{i \cdot j}{i \cdot i}}(-1)^{p} t_{i}^{-p\left(p^{\prime}-2 \frac{\langle i, j\rangle}{i \cdot i}+2 \frac{\langle j, i\rangle}{i \cdot i}\right)} F_{i}^{(p)} F_{j} F_{i}^{\left(p^{\prime}\right)}=0, \quad \text { if } i \neq j,
\end{aligned}
$$

where $E_{i}^{(p)}=\frac{E_{i}^{p}}{[p]_{i, t_{i}}^{!}}$. The algebra $U_{v, t}$ has a Hopf algebra structure with the comultiplication $\Delta$, the counit $\varepsilon$ and the antipode $S$ given as follows.

$$
\begin{array}{lll}
\Delta\left(K_{i}^{ \pm 1}\right)=K_{i}^{ \pm 1} \otimes K_{i}^{ \pm 1}, & \Delta\left(K_{i}^{ \pm 1}\right)=K_{i}^{\prime \pm 1} \otimes K_{i}^{\prime \pm 1}, & \\
\Delta\left(E_{i}\right)=E_{i} \otimes 1+K_{i} \otimes E_{i}, & \Delta\left(F_{i}\right)=1 \otimes F_{i}+F_{i} \otimes K_{i}^{\prime}, & \\
\varepsilon\left(K_{i}^{ \pm 1}\right)=\varepsilon\left(K_{i}^{\prime \pm 1}\right)=1, & \varepsilon\left(E_{i}\right)=\varepsilon\left(F_{i}\right)=0, & S\left(K_{i}^{ \pm 1}\right)=K_{i}^{\mp 1}, \\
S\left(K_{i}^{\prime \pm 1}\right)=K_{i}^{\prime \mp 1}, & S\left(E_{i}\right)=-K_{i}^{-1} E_{i}, & S\left(F_{i}\right)=-F_{i} K_{i}^{\prime-1} .
\end{array}
$$

This can be proved by checking the above relations (R1)-(R4). We refer to Chapter 3 in [26] for more details.

For any $\gamma=\left(\gamma_{1}, \gamma_{2}\right), \eta=\left(\eta_{1}, \eta_{2}\right) \in \mathbb{Z}^{I} \times \mathbb{Z}^{I}$, we define a bilinear form on $\mathbb{Z}^{I} \times \mathbb{Z}^{I}$ by

$$
[\gamma, \eta]^{\prime}=\left[\gamma_{2}, \eta_{2}\right]-\left[\gamma_{1}, \eta_{1}\right]
$$

The algebra $U_{v, t}$ admits a $\mathbb{Z}^{I} \times \mathbb{Z}^{I}$-grading by defining the degrees of generators as follows.

$$
\begin{aligned}
& \operatorname{deg}\left(E_{i}\right)=(i, 0), \quad \operatorname{deg}\left(K_{i}\right)=(i, i)=\operatorname{deg}\left(K_{i}^{\prime}\right), \\
& \operatorname{deg}\left(F_{i}\right)=(0, i), \quad \operatorname{deg}\left(K_{i}^{-1}\right)=(-i,-i)=\operatorname{deg}\left(K_{i}^{\prime-1}\right) .
\end{aligned}
$$

On $U_{v, t}$, we define a new multiplication "* $*$ by

$$
x * y=t^{-[|x|,|y|]^{\prime}} x y,
$$

for any homogenous elements $x, y \in U_{v, t}$. Since $[,]^{\prime}$ is a bilinear form, $\left(U_{v, t}, *\right)$ is an associative algebra over $\mathbb{Q}(v, t)$. We define a multiplication, denoted by “*”, on $U_{v, t} \otimes U_{v, t}$ by

$$
(x \otimes y) *\left(x^{\prime} \otimes y^{\prime}\right)=x * x^{\prime} \otimes y * y^{\prime} .
$$

This gives a new algebra structure on $U_{v, t} \otimes U_{v, t}$. $\left(U_{v, t}, *\right)$ has a Hopf algebra structure with the comultiplication $\Delta^{*}$, the counit $\varepsilon^{*}$ and the antipode $S^{*}$. The image of generators $E_{i}, F_{i}, K_{i}$ and $K_{i}^{-1}$ under the map $\Delta^{*}$ (resp. $\varepsilon^{*}$ and $S^{*}$ ) are the same as the ones under the map $\Delta$ (resp. $\varepsilon$ and $S$ ) defined in Section 4.2 . 
Under the new multiplication "*", the defining relations of $U_{v, t}$ in Section 4.2 can be rewritten as follows.

$$
\begin{array}{ll}
\left(R^{*} 1\right) \quad K_{i}^{ \pm 1} * K_{j}^{ \pm 1}=K_{j}^{ \pm 1} * K_{i}^{ \pm 1}, \quad K_{i}^{ \pm 1} * K_{j}^{\prime \pm 1}=K_{j}^{ \pm 1} * K_{i}^{\prime \pm 1}, \\
& K_{i}^{ \pm 1} * K_{j}^{ \pm 1}=K_{j}^{ \pm 1} * K_{i}^{ \pm 1}, \quad K_{i}^{ \pm 1} * K_{i}^{\mp 1}=1=K_{i}^{ \pm 1} * K_{i}^{\prime \mp 1} . \\
\left(R^{*} 2\right) \quad & K_{i} * E_{j} * K_{i}^{-1}=v^{i \cdot j} E_{j}, \quad K_{i}^{\prime} * E_{j} * K_{i}^{\prime-1}=v^{-i \cdot j} E_{j}, \\
& K_{i}^{\prime} * F_{j} * K_{i}^{\prime-1}=v^{i \cdot j} F_{j}, \quad K_{i} * F_{j} * K_{i}^{-1}=v^{-i \cdot j} F_{j} . \\
\left(R^{*} 3\right) \quad E_{i} * F_{j}-F_{j} * E_{i}=\delta_{i j} \frac{\widetilde{K}_{i}-\widetilde{K}_{i}^{\prime}}{v_{i}-v_{i}^{-1}, \quad \forall i, j \in I .} \\
\left(R^{*} 4\right) \quad \sum_{p+p^{\prime}=1-a_{i j}}(-1)^{p}\left[\begin{array}{c}
1-a_{i j} \\
p
\end{array}\right]_{v_{i}} E_{i}^{* p} * E_{j} * E_{i}^{* p^{\prime}}=0, \quad \text { if } i \neq j, \\
& \sum_{p+p^{\prime}=1-a_{i j}}(-1)^{p}\left[\begin{array}{c}
1-a_{i j} \\
p
\end{array}\right]_{v_{i}} F_{i}^{* p} * F_{j} * F_{i}^{* p^{\prime}}=0 \quad \text { if } i \neq j,
\end{array}
$$

where $a_{i j}=2 \frac{i \cdot j}{i \cdot i}$ and $E_{i}^{* p}=E_{i} * E_{i} * \cdots * E_{i}$ for $p$ copies. We notice that these relations are the specialization of (R1)-(R4) at $t=1$.

The one-parameter quantum algebra $U_{v}(I, \cdot)$ associated to $(I, \cdot)$ is the associative $\mathbb{Q}(v)$ algebra with 1 generated by symbols $E_{i}, F_{i}, K_{i}^{ \pm 1}, K_{i}^{\prime \pm 1}, \forall i \in I$ and subject to relations $\left(\mathrm{R}^{*} 1\right)-\left(\mathrm{R}^{*} 4\right) . U_{v}(I, \cdot)$ has a Hopf algebra structure with the comultiplication $\Delta_{1}$, the counit $\varepsilon_{1}$ and the antipode $S_{1}$. The image of generators $E_{i}, F_{i}, K_{i}$ and $K_{i}^{-1}$ under the map $\Delta_{1}$ (resp. $\varepsilon_{1}$ and $S_{1}$ ) are the same as the ones under the map $\Delta$ (resp. $\varepsilon$ and $S$ ) defined in Section 4.2 .

Let $U_{v, t}(I, \cdot):=U_{v}(I, \cdot) \otimes_{\mathbb{Q}(v)} \mathbb{Q}(v, t)$. The Hopf algebra structure on $U_{v}(I, \cdot)$ can be naturally extended to $U_{v, t}(I, \cdot)$. From the above analysis, we have the following theorem.

Theorem 4. If $(I, \cdot)$ is the Cartan datum associated to $\Omega$, then there is a Hopf-algebra isomorphism

$$
\left(U_{v, t}, *, \Delta^{*}, \varepsilon^{*}, S^{*}\right) \simeq\left(U_{v, t}(I, \cdot), \cdot, \Delta_{1}, \varepsilon_{1}, S_{1}\right),
$$

sending the generators in $U_{v, t}$ to the respective generators in $U_{v, t}(I, \cdot)$.

\section{The CANONICAL BASIS}

5.1. The canonical basis of $\mathfrak{f}$. Let $\mathfrak{A} \mathfrak{f}$ be the $\mathbb{N}^{I}$-graded $\mathfrak{A}$-subalgebra of $\mathfrak{f}$ generated by $\theta_{i}^{(n)}$ for various $i \in I$ and $n \in \mathbb{N}$. Let $\mathcal{B}$ be the subset of all elements $x$ in $\mathfrak{f}$ satisfying that

$$
x \in \mathfrak{A} \mathfrak{f}, \quad \bar{x}=x, \quad(x, x) \in 1+v^{-1} \mathbb{Z}\left[\left[v^{-1}\right]\right],
$$

where "-" is defined in Section 3.2 and (,) is defined in Proposition 13 .

Proposition 18. $\mathcal{B} \subset \mathbf{f} \otimes 1$.

Proof. For any $x \in \mathcal{B}, x$ can be written as $x=\sum_{b \in \mathbf{f} \otimes 1} b t^{n_{b}}$. Moreover, there are only finite nonzero summands. So $\max \left\{n_{b}\right\}$ exists, denoted by $n^{\prime}$. Therefore, $(x, x)=t^{2 n^{\prime}}$ plus lower power terms. Since $(x, x) \in 1+v^{-1} \mathbb{Z}\left[\left[v^{-1}\right]\right]$, we have $n^{\prime} \leq 0$. Similarly, let $n^{\prime \prime}=\min \left\{n_{b}\right\}$. Then $(x, x)=t^{2 n^{\prime \prime}}$ plus higher power terms. Since $(x, x) \in 1+v^{-1} \mathbb{Z}\left[\left[v^{-1}\right]\right]$, we have $n^{\prime \prime} \geq 0$. Therefore $n_{b}=0$ for all $b \in \mathbf{f} \otimes 1$. Proposition follows. 
Recall that a signed basis of an algebra $M$ is a subset, say $B$, of $M$ such that $B=B^{\prime} \cup\left(-B^{\prime}\right)$ for some basis $B^{\prime}$ of $M$.

Theorem 5. (a) $\mathcal{B}$ is a signed basis of the $\mathfrak{A}$-algebra $\mathfrak{A} \mathfrak{f}$ and that of the $\mathbb{Q}(v, t)$-algebra $\mathfrak{f}$; (b) $\left(b, b^{\prime}\right) \in \delta_{b b^{\prime}}+v^{-1} \mathbb{Z}\left[\left[v^{-1}\right]\right]$, for any $b^{\prime}, b \in \mathcal{B}$.

Proof. By Proposition 17, Proposition 18 and Theorem 14.2.3 in [26], Part (b) holds. Moreover, $\mathcal{B}$ is a signed basis of $\mathcal{A}$-module ${ }_{\mathcal{A}} \mathbf{f} \otimes 1$, where $\mathcal{A}=\mathbb{Z}\left[v^{ \pm 1}\right]$ and ${ }_{\mathcal{A}} \mathbf{f}$ is the $\mathcal{A}$-subalgebra of $\mathbf{f}$ generated by $\theta_{i}^{n} /[n]_{v_{i}}^{!}$. Since $\mathfrak{A} \mathfrak{f}=\left({ }_{\mathcal{A}} \mathbf{f} \otimes 1\right) \otimes_{\mathcal{A}} \mathfrak{A}$, Part (a) follows.

We call $\mathcal{B}$ the canonical signed basis of $\mathfrak{f}$.

For any $i \in I$ and $n \in \mathbb{Z}_{\geq 0}$, let $\mathcal{B}_{i, \geq n}=\mathcal{B} \bigcap \theta_{i}^{n} \mathfrak{f}$ and $\mathcal{B}_{i, n}=\mathcal{B}_{i, \geq n} \backslash \mathcal{B}_{i, \geq n+1}$. Then we have a parition $\mathcal{B}_{i, \geq n}=\coprod_{n^{\prime} \geq n} \mathcal{B}_{i, n^{\prime}}$.

Proposition 19. If $b \in \mathcal{B}_{i, 0}$, then there is a unique element $b^{\prime} \in \mathcal{B}_{i, n}$ such that $t^{-n[i,|b|]} \theta_{i}^{(n)} b=$ $b^{\prime}$ plus an $\mathfrak{A}$-linear combination of elements in $\mathcal{B}_{i, \geq n+1}$. Moreover, there is a bijection $\pi_{i, n}$ : $\mathcal{B}_{i, 0} \rightarrow \mathcal{B}_{i, n}$ sending $b$ to $b^{\prime}$.

Proof. By Proposition 18, Proposition 16 and Theorem 14.3.2(e) in [26], there is a unique 1-1 correspondence between $\mathcal{B}_{i, 0}$ and $\mathcal{B}_{i, n}$ such that $\frac{\theta_{i}^{* n}}{[n]_{v_{i}}} * b=b^{\prime}$ plus an $\mathcal{A}$-linear combination of elements in $\mathcal{B}_{i, \geq n+1}$, where $\theta_{i}^{* n}=\theta_{i} * \theta_{i} * \cdots * \theta_{i}$ for $n$ copies. By ([62) $) \theta_{i}^{(n)}=\frac{\theta_{i}^{* n}}{[n]_{v_{i}}}$ and $\frac{\theta_{i}^{* n}}{[n]_{v_{i}}^{n_{i}}} * b=t^{-n[i,|b|]} \theta_{i}^{(n)} b$. Proposition follows.

Given any $\nu \in \mathbb{N}^{I}$, we define a subset $\mathfrak{B}_{\nu}$ of $\mathcal{B}$ by induction on $\operatorname{tr}(\nu)$. Let $\mathfrak{B}_{0}=\{1\}$. If $\operatorname{tr}(\nu)>0$, we set

$$
\mathfrak{B}_{\nu}=\cup_{i \in I, n>0, \nu_{i} \geq n} \pi_{i, n}\left(\mathfrak{B}_{\nu-n i} \cap \mathcal{B}_{i, 0}\right),
$$

where $\pi_{i, n}$ is in Proposition 19, Let

$$
\mathfrak{B}=\sqcup_{\nu \in \mathbb{N} I} \mathfrak{B}_{\nu} .
$$

The following theorem is an analogue of Theorem 14.4.3 in [26].

Theorem 6. (a) $\mathcal{B}=\mathfrak{B} \cup(-\mathfrak{B})$;

(b) For any $\nu \in \mathbb{N}^{I}, \mathfrak{B}_{\nu} \cap\left(-\mathfrak{B}_{\nu}\right)=\varnothing$;

(c) For any $\nu \in \mathbb{N}^{I}, \mathfrak{B}_{\nu}$ is a basis of the $\mathfrak{A}$-algebra $\mathfrak{A f}_{\nu}$ and a basis of the $\mathbb{Q}(v, t)$-algebta $\mathfrak{f}_{\nu}$

(d) $\mathfrak{B}$ is a basis of the $\mathfrak{A}$-algebra $\mathfrak{A} \mathfrak{f}$ and a basis of the $\mathbb{Q}(v, t)$-algebra $\mathfrak{f}$.

Proof. By definition of $\pi_{i, n}$ and $\mathcal{B}_{i, \geq n}$, we have $\mathfrak{B} \cup(-\mathfrak{B}) \subset \mathcal{B}$. For any $\nu \in \mathbb{N}^{I}$ and any $x \in \mathcal{B}_{\nu}$, we are going to show that either $x \in \mathfrak{B}_{\nu}$ or $-x \in \mathfrak{B}_{\nu}$ by induction on $\operatorname{tr}(\nu)$. The case that $\operatorname{tr}(\nu)=0$ is trivial since $\mathfrak{B}_{0}=\{1\}$. Now assume that this statement is true for any $y \in \mathcal{B}$ with $\operatorname{tr}(|y|)<\operatorname{tr}(\nu)$.

Since we have a partition $\mathcal{B}=\sqcup_{n^{\prime} \geq 0} \mathcal{B}_{i, n^{\prime}}, x \in \mathcal{B}_{i, m}$ for some $m \geq 0$. By Proposition 19, there exists $x^{\prime} \in \mathcal{B}_{i, 0}$ such that $x=\pi_{i, m}\left(x^{\prime}\right)$. Moreover, $x^{\prime} \in \mathcal{B}_{\nu-m i}$. By induction assumption, either $x^{\prime} \in \mathfrak{B}_{\nu-m i}$ or $-x^{\prime} \in \mathfrak{B}_{\nu-m i}$. Therefore $x^{\prime} \in \mathfrak{B}_{\nu-m i} \cap \mathcal{B}_{i, 0}$ or $-x^{\prime} \in \mathfrak{B}_{\nu-m i} \cap \mathcal{B}_{i, 0}$. This implies that $x \in \mathfrak{B}_{\nu}$ or $-x \in \mathfrak{B}_{\nu}$. Part (a) follows.

Part (b) is trivial for $\operatorname{tr}(\nu)=0$. For any $x \in \mathfrak{B}_{\nu}$, by the definition of $\mathfrak{B}_{\nu}$, there exists $x^{\prime} \in \mathfrak{B}_{\nu-n i}$ for some $n \in \mathbb{N}$ and $i \in I$ such that $x=\pi_{i, n}\left(x^{\prime}\right)$. If $-x \in \mathfrak{B}_{\nu}$, then $-x^{\prime} \in \mathfrak{B}_{\nu-n i}$. This is a contradiction by an induction on $\operatorname{tr}(\nu)$. 
Since $\mathcal{B}$ is a signed basis, part (c) follows from part (a) and (b). Part (d) follows from part $(\mathrm{c})$.

Definition 1. The set $\mathfrak{B}$ defined in $(73)$ is called the canonical basis of $\mathfrak{f}$.

Recall that $\phi: \mathfrak{f} \rightarrow\left(\mathbf{f}_{v, t}, \odot\right)$ is the algebra isomorphism in Theorem 3 , Let $\mathfrak{B}(\phi)$ be the basis of $\mathfrak{f}$ such that the image of $\mathfrak{B}(\phi)$ under the map $\phi$ is the canonical basis of $\mathbf{f}_{v, t}$ defined in Theorem 14.4.3 in [26]. Both $\mathfrak{B}$ and $\mathfrak{B}(\phi)$ consist of elements in $\mathfrak{f}$ satisfying (172) by Propositions 17 and 18. Since $\mathfrak{B}_{0}=\{1\}=\mathfrak{B}_{0}(\phi)$, where $\mathfrak{B}_{0}$ is the subset of $\mathfrak{B}$ consisting of all degree 0 elements, by the uniqueness of $\mathfrak{B}$, we have the following corollary.

Corollary 4. $\mathfrak{B}(\phi)=\mathfrak{B}$. In other words, the canonical basis of $\mathfrak{f}$ is the same as that of $\mathbf{f}$ up to a 2-cocycle deformation. Moreover, if the associated Cartan data of $\Omega$ and $\Omega^{\prime}$ are the same, then the canonical bases of $\mathfrak{f}$ and $\mathfrak{f}\left(\Omega^{\prime}\right)$ are the same if we present both elements by the multiplication "*" in (62).

Example 3. Let $I=\{i\}$ and $\Omega_{i i}=1$, then $\mathfrak{B}=\left\{\theta_{i}^{(n)} \mid n \in \mathbb{N}\right\}$.

Example 4. Let $I=\{i, j\}$ and $\Omega_{i i}=\Omega_{j j}=1, \Omega_{i j}=-1, \Omega_{j i}=0$. For any $a, b, c \in \mathbb{N}$ such that $a+c \leq b$, we set

$$
\mathfrak{B}_{1}=\left\{t^{-a(b+c)} \theta_{i}^{(a)} \theta_{j}^{(b)} \theta_{i}^{(c)}\right\}, \quad \mathfrak{B}_{2}=\left\{t^{-a(b+c)} \theta_{j}^{(c)} \theta_{i}^{(b)} \theta_{j}^{(a)}\right\} .
$$

By Section 14.5.4 in [26], $\theta_{i}^{(a)} \theta_{j}^{(b)} \theta_{i}^{(c)}=\theta_{j}^{(c)} \theta_{i}^{(b)} \theta_{j}^{(a)}$ if $b=a+c$. By identifying these two elements, $\mathfrak{B}=\mathfrak{B}_{1} \cup \mathfrak{B}_{2}$.

5.2. The canonical basis of $L(\lambda, \epsilon)$. Let $U_{v, t}^{-}$be the negative part of $U_{v, t}$ generated by $F_{i}$ for all $i \in I$. As shown in Corollary 2, the algebra $U_{v, t}^{-}$can be identified with the algebra $\mathfrak{f}$ by sending the generator $F_{i}$ to $\theta_{i}$ for any $i \in I$. By abuse of notation, we denote by $\mathfrak{B}$ the image of the canonical basis in $\mathfrak{f}$ under the identification. For any pair $(\lambda, \epsilon) \in \mathbb{N}^{I} \times \mathbb{Q}(v, t)^{I}$ with $\epsilon \neq 0$, there exists a $U_{v, t}$-module $L(\lambda, \epsilon)$ containing a nonzero vector $\xi_{0} \in L(\lambda, \epsilon)$ and subject to

(a) $E_{i} \xi_{0}=0, K_{i} \xi_{0}=\epsilon_{i} v^{\lambda_{i}} \xi_{0}$ and $K_{i}^{\prime} \xi_{0}=\epsilon_{i} v^{-\lambda_{i}} \xi_{0}$ for all $i \in I$,

(b) The map $\varrho: U_{v, t}^{-} \rightarrow L(\lambda, \epsilon)$ given by $z \mapsto z \xi_{0}$ is surjective and its kernel is $\sum_{i \in I} U_{v, t}^{-} F_{i}^{\lambda_{i}+1}$. Let $\mathfrak{B}(\lambda, \epsilon)=\varrho\left(\mathfrak{B} \backslash\left(\left(\sum_{i \in I} U_{v, t}^{-} F_{i}^{\lambda_{i}+1}\right) \cap \mathfrak{B}\right)\right)$.

Proposition 20. (a) For any $\lambda \in \mathbb{N}^{I}$, the intersection $\left(\sum_{i \in I} \theta_{i}^{\lambda_{i}} \mathfrak{f}\right) \cap \mathfrak{B}$ is a $\mathbb{Q}(v, t)$-basis of $\sum_{i \in I} \theta_{i}^{\lambda_{i}} \mathfrak{f}$.

(b) For any $\lambda \in \mathbb{N}^{I}$, the intersection $\left(\sum_{i \in I} \mathfrak{f} \theta_{i}^{\lambda_{i}}\right) \cap \mathfrak{B}$ is a $\mathbb{Q}(v, t)$-basis of $\sum_{i \in I} \mathfrak{f} \theta_{i}^{\lambda_{i}}$.

Proof. By Corollary 11.8 in [25] and Theorem 3,

By Proposition 20 and the identification of $U_{v, t}^{-}$with $\mathfrak{f}$, we have that $\mathfrak{B}(\lambda, \epsilon) \subset L(\lambda, \epsilon)$ is a $\mathbb{Q}(v, t)$-basis of $L(\lambda, \epsilon)$.

Definition 2. $\mathfrak{B}(\lambda, \epsilon)$ is called the canonical basis of $L(\lambda, \epsilon)$. 
5.3. Positivity. Recall that to the matrix $\Omega$ of symmetric type, we have constructed an algebra $\mathfrak{f}$ in Section 3.2 and an algebra $\mathfrak{K}$ in Section 2.6 .

Theorem 7. The assignment $\theta_{i}^{(n)} \mapsto \mathfrak{L}_{n i}$ gives a twisted bialgebra isomorphism $\chi: \mathfrak{A} \mathfrak{f} \simeq$ $\mathfrak{K}$. Moreover, $\chi^{-1}(\widetilde{\mathfrak{B}})$ is the canonical basis of $\mathfrak{f}$ in Theorem $\underline{6}$, where $\widetilde{\mathfrak{B}}$ is the set of all isomorphism classes of simple perverse sheaves of weight 0 .

Proof. The proof of the first part is the same as the proof of Theorem 13.2.11 in [26]. We now show the second part. By Property 8.1.10 (d) in [26], (29) and (772), we have $\chi^{-1}(\widetilde{\mathfrak{B}}) \subset \mathcal{B}$. Let $\widetilde{\mathcal{B}}_{i, n}=\chi\left(\mathcal{B}_{i, n}\right)$, where $\mathcal{B}_{i, n}$ is defined in Section 5.1. For any $b \in \mathfrak{f}$, we write $\widetilde{b}=\chi(b)$. Let $\tilde{\pi}_{i, n}=\chi \pi_{i, n} \chi^{-1}$, where $\pi_{i, n}$ is defined in Proposition [19.

We claim that $\widetilde{\pi}_{i, n}: \widetilde{\mathcal{B}}_{i, 0} \rightarrow \widetilde{\mathcal{B}}_{i, n}$ preserves weights. In fact, for any $\widetilde{b} \in \widetilde{\mathcal{B}}_{i, 0}$, by Theorem 7 and Proposition 1, $\widetilde{\pi}_{i, n}(\widetilde{b})$ is a direct summand of $\mathfrak{I n d}_{n i,|b|}^{n i+|b|}\left(\mathfrak{L}_{n i} \otimes \widetilde{b}\left(-\frac{n[i,|b|]}{2}\right)\right)$. By Proposition 2, $\operatorname{wt}\left(\widetilde{\pi}_{i, n}(\widetilde{b})\right)=\operatorname{wt}(\widetilde{b})$.

By the construction of $\mathfrak{B}$, all complexes whose isomorphism classes are in $\chi^{-1}(\mathfrak{B})$ have weight 0 . Theorem follows.

From Theorem 14.4.13 in [26], Theorem 3 and Proposition 17, we have

Theorem 8. (Positivity) If $\Omega_{i i}=1$ for all $i \in I$, then we have

(a) $b b^{\prime}=\sum_{b^{\prime \prime} \in \mathfrak{B}, n \in \mathbb{Z}} c_{b, b^{\prime}, b^{\prime \prime}, n} v^{n} t^{\left[|b|,\left|b^{\prime}\right|\right]} b^{\prime \prime}$ such that $c_{b, b^{\prime}, b^{\prime \prime}, n} \in \mathbb{N}$ are zero except for finitely many $b^{\prime \prime}$ and $n$ for all $b, b^{\prime} \in \mathfrak{B}$;

(b) $r(b)=\sum_{b^{\prime}, b^{\prime \prime} \in \mathfrak{B}, n \in \mathbb{Z}} d_{b, b^{\prime}, b^{\prime \prime}, n} v^{n} t^{-\left[\left|b^{\prime}\right|,\left|b^{\prime \prime}\right|\right]} b^{\prime} \otimes b^{\prime \prime}$ such that $d_{b, b^{\prime}, b^{\prime \prime}, n} \in \mathbb{N}$ are zero except for finitely many $b^{\prime}, b^{\prime \prime}$ and $n$ for all $b \in \mathfrak{B}$;

(c) $\left(b, b^{\prime}\right)=\sum_{n \in \mathbb{N}} g_{b, b^{\prime}, n} v^{-n}$ such that $g_{b, b^{\prime}, n} \in \mathbb{N}$ for all $b, b^{\prime} \in \mathfrak{B}$.

The structure constants with respect to the canonical bases between $\mathfrak{f}$ and Lusztig's algebra $\mathbf{f}$ differ by a certain power of $t$ due to Theorem 3 . In particular, the specialization of the structure constants of $\mathfrak{f}$ with respect to $\mathfrak{B}$ at $t=1$ gives the structure constants of $\mathbf{f}$ with respect to the canonical basis of $\mathbf{f}$.

\section{A CATEgorification OF $\mathfrak{A} \mathfrak{f}$}

We shall give a categorification of $\mathfrak{A} \mathfrak{f}$ for arbitrary $\Omega$ based on a categorification of the integral form ${ }_{\mathcal{A}} \mathbf{f}$ of Lusztig's algebra $\mathbf{f}$. The followings are some examples of categorifications of ${ }_{\mathcal{A}} \mathbf{f}$.

Example 5. The triple $\left(\oplus_{\nu \in \mathbb{N}^{I}} \mathcal{Q}_{\nu}\right.$, Ind, Res) constructed in [26, Chapter 9] is a categorification of ${ }_{\mathcal{A}} \mathbf{f}$. Note that $\mathcal{Q}_{\nu}=\mathfrak{Q}_{\nu}^{\leq 0} \cap \mathfrak{Q}_{\nu}^{\geq 0}$.

Example 6. The triple $\left(\oplus_{\nu \in \mathbb{N} I} R_{\nu}\right.$-mod, Ind, Res) in [20] is a categorification of ${ }_{\mathcal{A}} \mathbf{f}$, where $R_{\nu}$-mod is a category of certain projective modules.

We fix a categorification $\left(\oplus_{\nu \in \mathbb{N}^{I}} \mathcal{Q}_{\nu}\right.$, Ind, Res) of $\mathcal{A}$-bialgebra ${ }_{\mathcal{A}} \mathbf{f}$. Given any $n \in \mathbb{Z}$, for each $\nu \in \mathbb{N}^{I}$, let $\mathcal{Q}_{n, \nu}$ be a category which is identical to $\mathcal{Q}_{\nu}$. We identify $\mathcal{Q}_{\nu}$ with $\mathcal{Q}_{0, \nu}$. For a fix $\nu \in \mathbb{N}^{I}$, the category $\mathcal{Q}_{n, \nu}$ are all identical to each other for different $n \in \mathbb{Z}$. Denote 
by $\mathcal{T}: \mathcal{Q}_{n-1, \nu} \rightarrow \mathcal{Q}_{n, \nu}$ the identity functor. We also denote by $\mathcal{T}^{n}: \mathcal{Q}_{k, \nu} \rightarrow \mathcal{Q}_{n+k, \nu}$ the composition functor of $\mathcal{T}$.

Let $\iota_{\nu}: \mathcal{Q}_{\nu} \rightarrow \oplus_{\nu} \mathcal{Q}_{\nu}$ and $p_{\nu}: \oplus_{\nu} \mathcal{Q}_{\nu} \rightarrow \mathcal{Q}_{\nu}$ be the natural embedding and projection functor, respectively. For any $\nu=\tau+\omega$, denote $\operatorname{Ind}_{\tau, \omega}^{\nu, 0}=p_{\nu} \circ \operatorname{Ind} \circ\left(\iota_{\tau} \times \iota_{\omega}\right)$ and $\operatorname{Res}_{\tau, \omega}^{\nu, 0}=$ $\left(p_{\tau} \times p_{\omega}\right) \circ$ Res $\circ \iota_{\nu}$. We define

$$
\begin{gathered}
\operatorname{Ind}_{\tau, \omega}^{\nu, n, m}: \mathcal{Q}_{n, \tau} \times \mathcal{Q}_{m, \omega} \rightarrow \mathcal{Q}_{n+m, \nu}, \quad(L, M) \mapsto \mathcal{T}^{n+m} \circ \operatorname{Ind}_{\tau, \omega}^{\nu, 0}\left(\mathcal{T}^{-n} L, \mathcal{T}^{-m} M\right) \text {, and } \\
\operatorname{Res}_{\tau, \omega}^{\nu, n, m}: \mathcal{Q}_{n+m, \nu} \rightarrow \mathcal{Q}_{n, \tau} \times \mathcal{Q}_{m, \omega}, \quad L \mapsto\left(\mathcal{T}^{n} \times \mathcal{T}^{m}\right) \circ \operatorname{Res}_{\tau, \omega}^{\nu, 0} \circ \mathcal{T}^{-(n+m)} L .
\end{gathered}
$$

Let $\mathfrak{Q}_{\nu}=\oplus_{n \in \mathbb{Z}} \mathcal{Q}_{n, \nu}$ and $\mathfrak{Q}=\oplus_{\nu \in \mathbb{N}_{I}} \mathfrak{Q}_{\nu}$. Define a $\mathbb{Z}\left[t^{ \pm 1}\right]$-action on the split Grothendieck group $K_{0}\left(\mathfrak{Q}_{\nu}\right)$ of $\mathfrak{Q}_{\nu}$ by

$$
t \cdot[L]=[\mathcal{T}(L)]
$$

where $[L]$ is the isomorphism class of $L$. Since $K_{0}\left(\mathcal{Q}_{n, \nu}\right)$ carries an $\mathcal{A}$-module structure for each pair $(n, \nu)$, the above action defines an $\mathfrak{A}$-module structure on $K_{0}\left(\mathfrak{Q}_{\nu}\right)$.

Given a functor $\mathfrak{F}$ between any two categories, we denote by $[\mathfrak{F}]$ the induced map between the corresponding Grothendieck groups. By (74) and (75), we have

$$
\left[\operatorname{Ind}_{\tau, \omega}^{\nu, n, m}\right] \circ\left(t^{n} \times t^{m}\right)=t^{n+m} \circ\left[\operatorname{Ind}_{\tau, \omega}^{\nu, 0}\right] \text {, and }\left(t^{n} \times t^{m}\right) \circ\left[\operatorname{Res}_{\tau, \omega}^{\nu, n, m}\right]=\left[\operatorname{Res}_{\tau, \omega}^{\nu, 0}\right] \circ t^{n+m} \text {. }
$$

For any $\nu=\tau+\omega$, we define functors

$$
\begin{gathered}
\mathfrak{I n d}_{\tau, \omega}^{\nu, n, m}: \mathfrak{Q}_{\tau} \times \mathfrak{Q}_{\omega} \rightarrow \mathfrak{Q}_{\nu}, \quad(L, M) \mapsto \mathcal{T}^{[\tau, \omega]} \circ \operatorname{Ind}_{\tau, \omega}^{\nu, n, m}(L, M), \text { and } \\
\mathfrak{R e s}_{\tau, \omega}^{\nu, n, m}: \mathfrak{Q}_{\nu} \rightarrow \mathfrak{Q}_{\tau} \times \mathfrak{Q}_{\omega}, \quad L \mapsto \mathcal{T}^{-[\tau, \omega]} \circ \operatorname{Res}_{\tau, \omega}^{\nu, n, m} L,
\end{gathered}
$$

where [,] is defined in (2). By assembling $\mathfrak{I n d}_{\tau, \omega}^{\nu, n, m}$ together, we have a functor $\mathfrak{I n d}: \mathfrak{Q} \otimes \mathfrak{Q} \rightarrow$ $\mathfrak{Q}$. Similarly, we have a functor $\mathfrak{R e s}=\bigoplus_{\tau+\omega=\nu} \mathfrak{R e s} \mathfrak{s}_{\tau, \omega}^{\nu, n, m}$.

Theorem 9. If ( $\mathcal{Q}$, Ind, Res) is a categorification of the $\mathcal{A}$-bialgebra ${ }_{\mathcal{A}} \mathbf{f}$, then $(\mathfrak{Q}, \mathfrak{I n d}, \mathfrak{R e s})$ is a categorification of the $\mathfrak{A}$-bialgebra $\mathfrak{A} \mathfrak{f}$.

Proof. Recall that the pair $(*, \widetilde{r})$ defined in (62) and (64) gives a new bialgebra structure on $\mathfrak{A} \mathfrak{f}$.

Since $\left(\mathcal{Q}\right.$, Ind, Res) is a categorification of ${ }_{\mathcal{A}} \mathbf{f}$, there exists a bialgebra isomorphism $\chi$ : ${ }_{\mathcal{A}} \mathbf{f} \rightarrow K_{0}(\mathcal{Q})$. Therefore, $\chi \otimes 1:{ }_{\mathcal{A}} \mathbf{f} \otimes_{\mathcal{A}} \mathfrak{A} \rightarrow K_{0}(\mathcal{Q}) \otimes_{\mathcal{A}} \mathfrak{A}$ is a bialgebra isomorphism. The bialgebra structure on ${ }_{\mathcal{A}} \mathbf{f} \otimes_{\mathcal{A}} \mathfrak{A}$ (resp. $K_{0}(\mathcal{Q}) \otimes_{\mathcal{A}} \mathfrak{A}$ ) can be obtained by field extension.

Recall that there is a bialgebra isomorphism $\rho:{ }_{\mathcal{A}} \mathbf{f} \otimes_{\mathcal{A}} \mathfrak{A} \rightarrow\left({ }_{\mathfrak{A}} \mathfrak{f}, *, \widetilde{r}\right)$ (see Proposition 16). Consider the $\mathfrak{A}$-linear map

$$
\psi: K_{0}(\mathcal{Q}) \otimes_{\mathcal{A}} \mathfrak{A} \rightarrow\left(K_{0}(\mathfrak{Q}),[\text { Ind }],[\text { Res }]\right), \quad L \otimes t^{n} \mapsto t^{n} \cdot L .
$$

We want to show that $\psi$ is a bialgebra isomorphism. It is a bijective map as an $\mathfrak{A}$-linear map. So it is enough to show that it is a bialgebra homomorphism. Firstly, $\psi$ is an algebra homomorphism, since

$$
\begin{aligned}
& \psi\left(\left(L \otimes t^{n}\right)\left(M \otimes t^{m}\right)\right)=\psi\left([\operatorname{Ind}](L, M) \otimes t^{n+m}\right)=t^{n+m} \cdot[\operatorname{Ind}](L, M) \\
& \quad=[\operatorname{Ind}]\left(t^{n} L, t^{m} M\right)=[\operatorname{Ind}]\left(\psi\left(L \times t^{n}\right), \psi\left(M \times t^{m}\right)\right) .
\end{aligned}
$$

Secondly, $\psi$ is a coalgebra homomorphism, because

$$
(\psi \times \psi)\left([\operatorname{Res}]\left(L \otimes t^{n}\right)\right)=(\psi \times \psi)\left([\operatorname{Res}](L) \otimes t^{n}\right)=\left(t^{n} \otimes 1\right) \cdot[\operatorname{Res}](L) .
$$


On the other hand, we have

$$
[\operatorname{Res}]\left(\psi\left(L \otimes t^{n}\right)\right)=[\operatorname{Res}]\left(t^{n} \cdot L\right)=\left(t^{n} \otimes 1\right) \cdot[\operatorname{Res}](L) .
$$

Therefore, we have the following diagram,

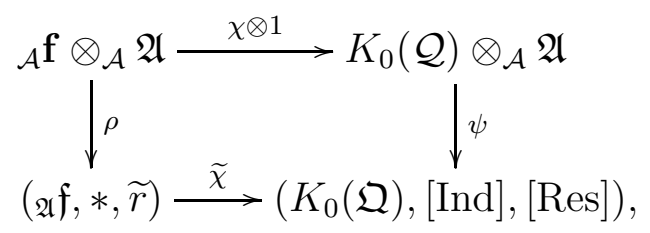

where $\tilde{\chi}=\psi \circ(\chi \otimes 1) \circ \rho^{-1}$. Since $\psi, \chi \otimes 1, \rho^{-1}$ are all bialgebra isomorphisms. This forces $\tilde{\chi}$ to be also a bialgebra isomorphism.

Lastly, we show that $\widetilde{\chi}: \mathfrak{A} \mathfrak{f} \rightarrow\left(K_{0}(\mathfrak{Q}), \mathfrak{I n} \mathfrak{d}, \mathfrak{R e s}\right)$ is also a bialgebra isomorphism. As a $\mathfrak{A}-$ linear map, $\tilde{\chi}$ is a bijective map. So it is enough to show that $\tilde{\chi}$ is a bialgebra homomorphism. $\tilde{\chi}$ is an algebra homomorphism, since, for any homogeneous elements $L, M \in \mathfrak{A} \mathfrak{f}$, we have

$$
\widetilde{\chi}(L M)=t^{[|L|,|M|]} \widetilde{\chi}(L * M)=t^{[|L|,|M|]} \operatorname{Ind}(\widetilde{\chi}(L), \widetilde{\chi}(M))=\mathfrak{I n d}(\widetilde{\chi}(L), \widetilde{\chi}(M)) .
$$

For any $L \in \mathfrak{A} \mathfrak{f}$, let us write $\widetilde{r}(L)=\sum L_{1} \otimes L_{2}$. Then we have,

$$
\widetilde{\chi}(r(L))=\widetilde{\chi}\left(t^{-\left[\left|L_{1}\right|,\left|L_{2}\right|\right]} L_{1} \otimes L_{2}\right)=\sum t^{-\left[\left|L_{1}\right|,\left|L_{2}\right|\right]}\left(\widetilde{\chi}\left(L_{1}\right) \otimes \widetilde{\chi}\left(L_{2}\right)\right)=\mathfrak{R e s}(\widetilde{\chi}(L)) .
$$

This finishes the proof.

\section{REFERENCES}

[1] A. A. Beĭlinson, J. Bernstein, and P. Deligne, Faisceaux pervers, Analysis and topology on singular spaces, I (Luminy, 1981), Astérisque, vol. 100, Soc. Math. France, Paris, 1982, pp. 5-171.

[2] G. Benkart and S. Witherspoon, Representations of two-parameter quantum groups and Schur-Weyl duality, Hopf algebras, Lecture Notes in Pure and Appl. Math., vol. 237, Dekker, New York, 2004, pp. 65-92.

[3] N. Bergeron, Y. Gao, and N. Hu, Representations of two-parameter quantum orthogonal and symplectic groups, Proceedings of the International Conference on Complex Geometry and Related Fields (Providence, RI), AMS/IP Stud. Adv. Math., vol. 39, Amer. Math. Soc., 2007, pp. 1-21.

[4] T. Braden, Hyperbolic localization of intersection cohomology, Transform. Groups 8 (2003), no. 3, 209-216.

[5] S. Clark, Z. Fan, Y. Li, and W. Wang, Quantum supergroups III. Twistors, submitted. arXiv:1307.7056.

[6] L. Crane and I. B. Frenkel, Four-dimensional topological quantum field theory, Hopf categories, and the canonical bases, J. Math. Phys. 35 (1994), no. 10, 5136-5154, Topology and physics.

[7] V. Dobrev, Duality for the matrix quantum group GLp,q(2,C). J. Math. Phys. 33 (1992), no. 10, 3419-3430.

[8] J. Duncan, P. Etingof, I. Ip, M. Khovanov, M. Libine, A. Licata, A. Savage, and M. Schlosser, On the work of Igor Frenkel, at http://www.math.sunysb.edu/frenkel60/Frenkel/Poster2/igorwork.pdf (2012).

[9] S. Fomin and A. Zelevinsky, Cluster algebras. I. Foundations, J. Amer. Math. Soc. 15 (2002), no. 2, 497-529 (electronic).

[10] E. Freitag and R. Kiehl, Étale cohomology and the Weil conjecture, Ergebnisse der Mathematik und ihrer Grenzgebiete (3) [Results in Mathematics and Related Areas (3)], vol. 13.

[11] E. Frenkel and D. Hernandez, Langlands duality for representations of quantum groups, Math. Ann. 349 (2011), no. 3, 705-746.

[12] Christian Frønsdal, q-algebras and arrangements of hyperplanes, J. Algebra 278 (2004), no. 2, 433455 . 
[13] I. Grojnowski and G. Lusztig, A comparison of bases of quantized enveloping algebras, Linear algebraic groups and their representations (Los Angeles, CA, 1992), Contemp. Math., vol. 153, Amer. Math. Soc., Providence, RI, 1993, pp. 11-19.

[14] D. Hill and W. Wang, Categorification of quantum Kac-Moody superalgebras, Transactions of AMS, to appear. arXiv:1202.2769 (2012).

[15] R. Hotta and M. Kashiwara, The invariant holonomic system on a semisimple Lie algebra, Invent. Math. 75 (1984), no. 2, 327-358.

[16] N. Hu and Y. Pei, Notes on Two-Parameter Quantum Groups,(II), Communications in Algebra 40 (2012), no. 9, 3202-3220.

[17] N. Hu, Y. Pei, and M. Rosso, Multi-parameter quantum groups and quantum shuffles. I, Quantum affine algebras, extended affine Lie algebras, and their applications, Contemp. Math., vol. 506, Amer. Math. Soc., Providence, RI, 2010, pp. 145-171.

[18] N. Jing and H. Zhang, Two-parameter quantum vertex representations via finite groups and the McKay correspondence, Trans. Amer. Math. Soc. 363 (2011), no. 7, 3769-3797.

[19] M. Kashiwara, On crystal bases of the Q-analogue of universal enveloping algebras, Duke Math. J. 63 (1991), no. 2, 465-516.

[20] M. Khovanov and A. D. Lauda, A diagrammatic approach to categorification of quantum groups. I, Represent. Theory 13 (2009), 309-347.

[21] M. Khovanov and A. D. Lauda, A diagrammatic approach to categorification of quantum groups II, Trans. Amer. Math. Soc. 363 (2011), no. 5, 2685-2700.

[22] C. Korff and C. Stroppel, The sl(n)-WZNW fusion ring: a combinatorial construction and a realisation as quotient of quantum cohomology, arXiv:0909.2347.

[23] G. Lusztig, Character sheaves. I, Adv. in Math. 56 (1985), no. 3, 193-237.

[24] G. Lusztig, Canonical bases arising from quantized enveloping algebras, J. Amer. Math. Soc. 3 (1990), no. $2,447-498$.

[25] G. Lusztig, Quivers, perverse sheaves, and quantized enveloping algebras, J. Amer. Math. Soc. 4 (1991), no. 2, 365-421.

[26] G. Lusztig, Introduction to quantum groups, Modern Birkhäuser Classics, Birkhäuser/Springer, New York, 2010.

[27] M. Reineke, Generic extensions and multiplicative bases of quantum groups at $q=0$, Represent. Theory 5 (2001), 147-163 (electronic).

[28] M. Takeuchi, A two-parameter quantization of $G L(n)$ (summary), Proc. Japan Acad. Ser. A Math. Sci. 66 (1990), no. 5, 112-114.

Department of Mathematics, University at Buffalo, SUNY, 244 Mathematics Building, BufFALO, NY 14260

E-mail address: zhaobing@buffalo.edu (Z.Fan), yiqiang@buffalo.edu (Y.Li) 